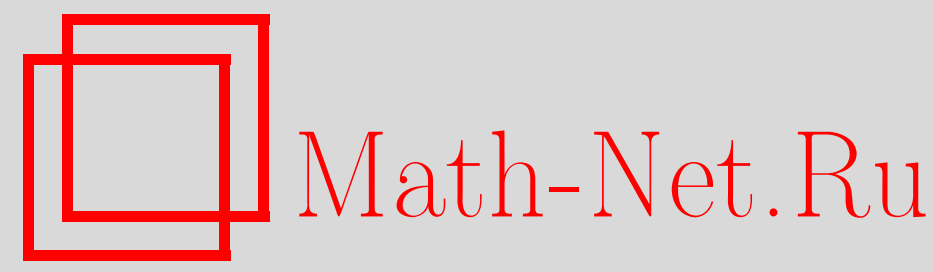

Ю. Н. Дрожжинов, Б. И. Завьялов, Многомерные тауберовы теоремы для обобщенных функций со значениями в банаховых пространствах, Матем. сб., 2003, том 194, номер 11, 17-64

DOI: https://doi.org/10.4213/sm779

Использование Общероссийского математического портала Math-Net.Ru подразумевает, что вы прочитали и согласны с пользовательским соглашением

http://www. mathnet.ru/rus/agreement

Параметры загрузки:

IP : 54.172 .240 .79

26 апреля 2023 г., 12:28:18 


\title{
Многомерные тауберовы теоремы для обобщенных функций со значениями в банаховых пространствах
}

\begin{abstract}
В работе формулируются и доказьваются многомерные тауберовы теоремы для стандартных усреднений обобщенных функций медленного роста со значениями в банаховых пространствах. Эти теоремы позволяют по асимптотическому поведению усреднений судить об асимптотическом поведении самой усредняемой функции. Асимптотической шкалой в этих теоремах служит класс правильно меняющихся функций. Особое внимание уделено ядрам усреднения, некоторые из моментов которых или их линейные комбинации обращаются в нуль. Существенную роль в этих теоремах играет структура нуля преобразований Фурье рассматриваемых ядер.

Доказанные теоремы применяются для изучения асимптотических свойств решений задачи Коши для уравнения теплопроводности в классе медленно растущих обобщенных функций, к задаче о диффузии многокомпонентного газа и к задаче о некомпенсации особенностей для голоморфных функций в трубчатых областях над острьми конусами.

Библиограффия: 6 названий.
\end{abstract}

\section{Введение}

В предыдущих статьях [1], [2] мы исследовали условия, при которых обобщенная функция одного переменного, принимающая а priori значения в локально выпуклом пространстве, на самом деле, принимает значения в более узком банаховом пространстве. Эти условия формулировались в терминах свойств стандартных усреднений обобщенных функций с невырожденными ядрами. В настоящей работе мы рассматриваем аналогичные вопросы в случае обобщенных функций многих переменных. При этом если рассматривать ядра усреднений, нулевой момент которых отличен от нуля, то ситуация здесь принципиально не отличается от одномерной, а возникающие дополнительные трудности носят чисто технический характер. В многомерном случае большой интерес представляют ядра усреднений, некоторые из моментов которых или их линейных комбинаций обращаются в нуль. Тогда ситуация становится существенно сложнее и, вместе с тем, значительно богаче. Это, в частности, связано со сложной структурой нулей многочленов нескольких переменных.

В $\S 1$ мы вводим основные определения и обозначения и напоминаем некоторые факты из теории обобщенных функций и смежных областей.

Работа вьполнена при финансовой поддержке Российского фонда фундаментальных исследований (грант № 02-01-00076) и Программы поддержки ведущих научных школ (грант № НШШ-1542.2003.1).

(С) Ю.Н. Дрожжинов, Б. И. ЗАвьялов 2003 
Целью $\S 2$ является доказательство общего утверждения, состояшего в том, что если некоторое усреднение заданной обобшенной функции $f$, принимающей значения в некотором широком банаховом пространстве $\mathscr{L}$, принимает значения в более узком банаховом пространстве $\mathbb{B}$ и удовлетворяет там стандартной "оценке класса", то и сама $f$ принимает значения в $\mathbb{B}$ на некотором замкнутом подпространстве $\mathscr{S}_{1}$ пространства основных функций $\mathscr{S}$. Это подпространство строится с помощью некоторых идеалов кольца всех многочленов $n$ переменных, которые, в свою очередь, определяются ядром усреднения. Доказательство этого утверждения опирается на несколько лемм, некоторые из которых представляют и самостоятельный интерес.

В $\S 3$ это общее утверждение применяется в конкретных, но важных случаях, связанных с квазиасимптотическим поведением обобщенных функций. При этом возникают новые интересные эффекты, существенно уточняющие общие результаты. В частности, оказалось, что обобшенная функция, которая согласно общему утверждению обладает квазиасимптотикой лишь на замкнутом подпространстве основных функций, будет обладать квазиасимптотикой на всем $\mathscr{S}$, если из нее предварительно вычесть контрчлены специального вида.

В $\S 4$ мы применяем полученные результаты для изучения асимптотических свойств решений задачи Коши для уравнения теплопроводности, задачи Коши для диффузии многокомпонентных веществ и некомпенсации граничных особенностей голоморфных функций в трубчатых областях.

В приложение мы отнесли несколько лемм, одна из которых является некоторым вариантом классической теоремы Карлесона о короне. Доказательство этих лемм в основном тексте сильно перегрузило бы изложение и затруднило восприятие основных результатов.

\section{§1. Основные обозначения и определения}

Через $\mathscr{S}$ обозначаем пространство бесконечно дифференциируемых функций $\varphi(x), x \in \mathbb{R}^{n}$, быстроубываюших вместе со всеми производными, с топологией, задаваемой счетной системой полунорм

$$
\mathscr{P}_{N}[\varphi]=\max _{0 \leqslant|j| \leqslant N} \sup _{x \in \mathbb{R}^{n}}\left(1+|x|^{2}\right)^{N / 2}\left|\varphi^{(j)}(x)\right|, \quad N=0,1, \ldots
$$

Здесь $j$ - мультиндекс. Как обычно,

$$
\begin{gathered}
j=\left(j_{1}, \ldots, j_{n}\right), \quad|j|=j_{1}+j_{2}+\cdots+j_{n}, \quad x^{j}=x_{1}^{j_{1}} \cdot \ldots \cdot x_{n}^{j_{n}} \\
\partial x^{j}=\partial x_{1}^{j_{1}} \cdot \partial x_{2}^{j_{2}} \cdot \ldots \cdot \partial x_{n}^{j_{n}}, \quad j !=j_{1} ! \cdot j_{2} ! \cdot \ldots \cdot j_{n} !, \quad \varphi^{(j)}(x)=\frac{\partial^{|j|}}{\partial x^{j}} \varphi(x) .
\end{gathered}
$$

Через $\mathscr{S}_{N}$ будем обозначать банахово пространство с нормой $\mathscr{P}_{N}$, так что $\mathscr{S}=$ $\bigcap_{N \geqslant 0} \mathscr{S}_{N}$

Пространство $\mathscr{S}^{\prime}$ - сопряженное к пространству $\mathscr{S}$ (пространство обобщенных функций медленного роста).

Всюду далее волной обозначается преобразование Фурье основной (обобшенной) функции, так что если $\varphi(x) \in \mathscr{S}$, то

$$
F[\varphi]=\widetilde{\varphi}(t)=\int e^{i x t} \varphi(x) d x .
$$


Напомним, что $F[\mathscr{S}]=\mathscr{S}$. Для обобщенных функций по определению $(\widetilde{f}, \widetilde{\varphi})=$ $(f, \varphi)$.

Как обычно, через $[x]$ обозначаем целую часть числа $x$.

Тейлоровское разложение в начале координат функции $\varphi(x) \in C^{\infty}\left(\mathbb{R}^{n}\right)$ до порядка $\ell$ включительно обозначаем $T_{\varphi}^{\ell}(x)$, так что

$$
T_{\varphi}^{\ell}(x)=\sum_{|j|=0}^{\ell} \frac{1}{j !} \varphi^{(j)}(0) x^{j}
$$

ОПРЕДЕЛЕНИЕ 1. Будем говорить, что функция $\varphi(x) \in \mathscr{S}$ не вырождена в нуле, если сушествует число $m$ такое, что ее тейлоровское разложение порядка $m$ в начале координат удовлетворяет следующему условию:

для любого вектора $\bar{e} \in \mathbb{R}^{n},|\bar{e}|=1$,

$$
T_{\varphi}^{m}(\lambda \bar{e}) \not \equiv 0 \quad \text { при } \lambda>0 .
$$

Через $\mathbb{P}$ обозначаем кольцо всех многочленов от $n$ переменных. Пусть $\ell$ - целое неотрицательное число. Через $\mathbb{P}^{\ell}$ обозначим кольцо (идеал в $\mathbb{P}$ ) всех многочленов вида $\sum_{|j|=\ell+1}^{q} C_{j} x^{j}$, здесь $q-$ любое целое число, $\ell+1 \leqslant q<\infty$.

Пусть $\left\{P_{0}(x), P_{1}(x), \ldots, P_{N}(x)\right\}$ - множество многочленов, где $P_{k}$ - однородный многочлен степени $k$. Через $\mathbb{P}\left[P_{0}, P_{1}, \ldots, P_{N}\right]$ обозначаем идеал $($ в $\mathbb{P})$, натянутьй на многочлены $P_{k}(x), k=0,1, \ldots, N$. Другими словами, многочлен $T^{\ell}(t)$ степени $\ell$ принадлежит идеалу $\mathbb{P}\left[P_{0}, P_{1}, \ldots, P_{N}\right]$, если

$$
T^{\ell}(t)=\sum_{k=0}^{N} P_{k}(t) Q_{k}(t)
$$

где без ограничения общности можно считать, что $Q_{k}(t)$ - многочлены степени не выше $\ell-k$, в частности, $N \leqslant \ell$. Отметим, что представление (1.2) неоднозначно. Однако, пользуясь стандартными методами линейной алгебры, можно доказать следуюшее утверждение.

Обозначим через $\bar{Q}$ вектор, имеющий своими компонентами все коэффициенты многочленов $Q_{k}(t), k=0, \ldots, N$, а через $\bar{T}$ - вектор, имеюший своими компонентами все коэффициенты многочлена $T^{M}(t)$. Тогда существует постоянная $A$, зависящая только от $\ell$ и многочленов $P_{k}(t), k=0, \ldots, N$, такая, что многочлены $Q_{k}(t)$ в (1.2) могут быть выбраны таким образом, чтобы выполнялась оценка

$$
\|\bar{Q}\| \leqslant A\|\bar{T}\|
$$

ОПРЕДЕЛЕНИЕ 2. Пусть $M$ - целое число и $\left\{P_{k}(x), k=0,1, \ldots, N\right\}$ - множество, где $P_{k}(x)$ - однородньй многочлен степени $k$ или тождественный нуль. Через $\mathscr{S}_{\left[M ; P_{0}, \ldots, P_{N}\right]}$ обозначим подпространство пространства $\mathscr{S}$, состоящее из основных функций, тейлоровское разложение которых в начале координат порядка $M$ принадлежит идеалу $\mathbb{P}\left[P_{0}, P_{1}, \ldots, P_{N}\right]$. Другими словами,

$$
\mathscr{S}_{\left[M ; P_{0}, \ldots, P_{N}\right]}=\left\{\varphi \in \mathscr{S}: T_{\varphi}^{M} \in \mathbb{P}\left[P_{0}, P_{1}, \ldots, P_{N}\right]\right\}
$$


При этом условимся считать, что при $M<0$ справедливо $\mathscr{S}_{\left[M ; P_{0}, \ldots, P_{N}\right]}=\mathscr{S}$. Заметим, что если $N>M$, то

$$
\mathscr{S}_{\left[M ; P_{0}, \ldots, P_{N}\right]}=\mathscr{S}_{\left[M ; P_{0}, \ldots, P_{M}\right]} \cdot
$$

Положим далее

$$
\widetilde{\mathscr{S}}_{\left[M ; P_{0}, \ldots, P_{N}\right]}=\left\{\varphi \in \mathscr{S}: \widetilde{\varphi} \in \mathscr{S}_{\left[M ; P_{0}, \ldots, P_{N}\right]}\right\} .
$$

Отметим, что если $P_{0} \neq 0$, то $\mathscr{S}_{\left[M ; P_{0}, \ldots, P_{N}\right]}=\mathscr{S}$.

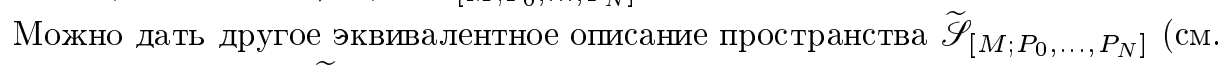

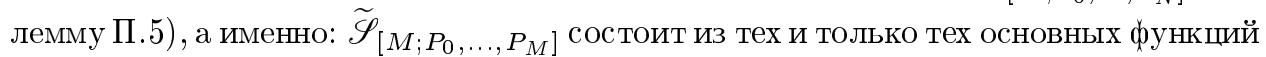
$\varphi(x) \in \mathscr{S}$, для которых $\int Q(x) \varphi(x) d x=0$, где $Q(x)$ - любой многочлен степени не вьше $M$ такой, что

$$
P_{q}\left(\frac{\partial}{\partial x}\right) Q(x)=0, \quad q=0,1, \ldots, M
$$

Отсюда, в частности, следует, что $\tilde{\mathscr{S}}_{\left[M ; P_{0}, \ldots, P_{M}\right]}$ (а, следовательно, и $\left.\mathscr{S}_{\left[M ; P_{0}, \ldots, P_{M}\right]}\right)$ - замкнутое подпространство пространства $\mathscr{S}$.

Пусть $\mathscr{F}$ и $\mathscr{L}$ - произвольные локально вьпуклые топологические пространства. Через $\mathbb{L}(\mathscr{F} \rightarrow \mathscr{L})$ обозначаем пространство всех линейных непрерывных отображений из $\mathscr{F}$ в $\mathscr{L}$. Таким образом, $\mathscr{S}^{\prime}$ есть не что иное как $\mathbb{L}(\mathscr{S} \rightarrow \mathbb{C})$. Пространство $\mathbb{L}(\mathscr{S} \rightarrow \mathscr{L})$ можно трактовать как пространство обобшенных функций медленного роста над $\mathscr{S}$ со значениями в $\mathscr{L}$. Для $f \in \mathbb{L}(\mathscr{S} \rightarrow \mathscr{L})$ и $\varphi \in \mathscr{S}$ положим

$$
L_{f}^{\varphi}(x, y)=\left(f(\xi), \frac{1}{y^{n}} \varphi\left(\frac{x-\xi}{y}\right)\right), \quad(x, y) \in \mathbb{R}^{n} \times(0,1],
$$

$L_{f}^{\varphi}(x, y)$ - это стандартное усреднение обобщенной функции $f$ с ядром $\varphi$. Отметим его связь с обычным преобразованием Лапласа. Пусть $\Gamma$ - острьй выпуклый замкнутый конус в $\mathbb{R}^{n}$ с вершиной в начале координат и $C=\operatorname{int} \Gamma^{*}$, где $\Gamma^{*}$ - сопряженный конус (см., например, [3]). Пусть supp $\widetilde{f} \subset \Gamma$. Преобразование Лапласа $\widetilde{f}$ определяется формулой

$$
L[\widetilde{f}](z)=\left(\widetilde{f}(t), e^{i(z, t)}\right), \quad \operatorname{Im} z \in \mathbb{C} .
$$

Нетрудно видеть, что если $\widetilde{\omega}(t) \in \mathscr{S}$ и $\widetilde{\omega}(t)=e^{-(h, t)}$ при $h \in C$ и $t \in \Gamma$, то

$$
L[\widetilde{f}](x+i y h)=L_{f}^{\omega}(x, y), \quad x \in \mathbb{R}^{n}, \quad y>0 .
$$

Таким образом, $L_{f}^{\omega}(x, y)$ при произвольном $\omega \in \mathscr{S}$ можно считать обобщенныц преобразованием Лапласа от $\widetilde{f} \in \mathscr{S}^{\prime}$.

Если $\mathbb{B}$ - банахово пространство, следующее утверждение является прямьм следствием непрерьвности отображения $f$ на пространстве $\mathscr{S}$.

Пусть $f(\xi) \in \mathbb{L}(\mathscr{S} \rightarrow \mathbb{B})$. Тогда сушествуют $C, N, a$ и $b$ такие, что

$$
\left\|L_{f}^{\varphi}(x, y)\right\|_{\mathbb{B}} \leqslant \frac{C}{y^{b}}(1+|x|)^{a} P_{N}[\varphi], \quad 0<y \leqslant 1, \quad x \in \mathbb{R}^{n}, \quad \forall \varphi \in \mathscr{S} .
$$


Пусть функция $g(x)$ определена на некотором открытом множестве $\Omega \subset \mathbb{R}^{n}$ и принимает значения в некотором банаховом пространстве $\mathbb{B}$. Мы говорим, что $g(x)$ измерима, если она измерима по Бохнеру, т.е. если она является пределом почти всюду по $x \in \Omega$ последовательности непрерывных в $\Omega$ функций со значениями в $\mathbb{B}$.

Пусть $\rho(k)$ - положительная и непрерьвная функция при $k \geqslant k_{0}$, где $k_{0}-$ некоторое положительное число. Говорят, что $\rho(k)$ - автомодельная (правильно меняющаяся) функиия, если существует предел

$$
\lim _{k \rightarrow+\infty} \frac{\rho(k \xi)}{\rho(k)}=\psi(\xi)
$$

причем сходимость равномерна по $\xi$ на каждом компакте полуоси $(0, \infty)$. Легко видеть, что $\psi(\xi)=\xi^{\alpha}$ при некотором $\alpha$. В этом случае функцию $\rho(k)$ называют автомодельной порядка $\alpha$.

Ясно, что автомодельную функцию можно доопределить на отрезке $\left[0, k_{0}\right]$ так, чтобы она стала непрерывной и положительной на всей замкнутой положительной полуоси. В дальнейшем мы полагаем, что любая автомодельная функция определена, непрерывна и положительна на $[0,+\infty)$. При этом для любого $\varepsilon>0$ сушествует $C_{\varepsilon}$ такая, что

$$
\frac{\rho(\varkappa k)}{\rho(k)} \leqslant C_{\varepsilon} \begin{cases}\left(1+\varkappa^{\alpha+\varepsilon}\right), & k>1, \alpha>0 \\ \varkappa^{\alpha \pm \varepsilon}, & k>1, \alpha \leqslant 0\end{cases}
$$

где верхний знак берется при $\varkappa \geqslant 1$ и нижний - при $\varkappa<1$. Подробнее см. [3], [4]. Кроме того, для автомодельных функций при $k \rightarrow+\infty$ справедливы соотношения

$$
k \rho(k) \sim\left\{\begin{array}{l}
\int_{1}^{k} \rho(\varkappa) d \varkappa, \quad \alpha>-1 \\
\int_{k}^{\infty} \rho(\varkappa) d \varkappa, \quad \alpha<-1 .
\end{array}\right.
$$

ОПРЕДЕЛЕНИЕ 3 . Пусть $\mathbb{B}$ - банахово пространство, $f \in \mathbb{L}(\mathscr{S} \rightarrow \mathbb{B}), \rho(k)$ - автомодельная функция и $E$ - некоторое множество основных функций из $\mathscr{S}$. Будем говорить, что $f$ квазиасимптотически ограничена (обладает квазиасимптотикой) в нуле относительно $\rho(k)$ на множестве $E \subset \mathscr{S}$, если

$$
\sup _{k>1} \frac{1}{\rho(k)}\left\|\left(f\left(\frac{x}{k}\right), \varphi(x)\right)\right\|_{\mathbb{B}}<\infty \quad\left(\lim _{k \rightarrow+\infty} \frac{1}{\rho(k)}\left(f\left(\frac{x}{k}\right), \varphi(x)\right)=C_{\varphi}\right)
$$

для любой $\varphi \in E$. Аналогично, будем говорить, что $f$ квазиасимптотически ограничена (обладает квазиасимптотикой) на бесконечности относительно $\rho(k)$ на множестве $E \subset \mathscr{S}$, если

$$
\sup _{k>1} \frac{1}{\rho(k)}\|(f(k x), \varphi(x))\|_{\mathbb{B}}<\infty \quad\left(\lim _{k \rightarrow+\infty} \frac{1}{\rho(k)}(f(k x), \varphi(x))=C_{\varphi}\right)
$$

для любой $\varphi \in E$. 


\section{§2. Основные леммы и утверждения}

Основная цель этого параграфа - доказать теорему 2.1. Этому доказательству мы предпошлем несколько лемм, касаюшихся стандартных усреднений обобщенных функций над $\mathscr{S}$ со значениями в локально выпуклом пространстве $\mathscr{L}$. Напомним, что усреднение функции $f \in \mathbb{L}(\mathscr{S} \rightarrow \mathscr{L})$ с ядром $\omega \in \mathscr{S}$ задается формулой

$$
L_{f}^{\omega}(x, y)=\left(f(\xi), \frac{1}{y^{n}} \omega\left(\frac{x-\xi}{y}\right)\right), \quad(x, y) \in \mathbb{R}^{n} \times(0,1]
$$

Очевидно, что $L_{f}^{\omega}(x, y)$ - непрерывная функция в $\mathbb{R}^{n} \times(0,1]$ со значениями в $\mathscr{L}$. Далее в этом параграфе будет типична следующая ситуация.

Пусть $f \in \mathbb{L}(\mathscr{S} \rightarrow \mathscr{L})$ и $\mathbb{B}$ - банахово пространство, причем, $\mathbb{B} \subset \mathscr{L}$. Будем говорить, что для некоторого ядра $\omega(x) \in \mathscr{S}$ усреднение $L_{f}^{\omega}(x, y)$ удовлетворяет условиям а) и b) соответственно, если:

а) при каждом $y \in(0,1]$ функция $L_{f}^{\omega}(x, y)$ принимает значение в $\mathbb{B}$ при почти всех $x \in \mathbb{R}^{n}$ и измерима по $x$ как функция со значениями в $\mathbb{B}$;

b) сушествуют постоянные $C, a$ и $b$ такие, что при $0<y \leqslant 1$ имеет место неравенство

$$
\left\|L_{f}^{\omega}(x, y)\right\|_{\mathbb{B}} \leqslant C \frac{(1+|x|)^{a}}{y^{b}} \text { при п.в. } x \in \mathbb{R}^{n} .
$$

ЛЕмма 2.1. Пусть $\mathbb{B}$ - банахово и $\mathscr{L}$ - локально выпуклое пространства, причем $\mathbb{B} \subset \mathscr{L}$ линейно и непрерьвно. Пусть еще $f \in \mathbb{L}(\mathscr{S} \rightarrow \mathscr{L})$ u $\omega(x) \in \mathscr{S}$ такова, что ее преобразование Фурве $\widetilde{\omega}(t)=P(t) \widetilde{\omega}_{1}(t)$, где $\widetilde{\omega}_{1}(t) \in \mathscr{S}$, $\widetilde{\omega}_{1}(0) \neq 0$, а $P(t)=\sum_{k=0}^{N} P_{k}(t)$, где $P_{k}(t)$ - однородныи многочлен $n$ переменных степени $k$ либо тождественный нуль. Предположсим такжсе, что для функиии $L_{f}^{\omega}(x, y)$ выполнень условия а) и b). Тогда

$$
(f(x), \varphi(x)) \in \mathbb{B}
$$

для всех основных функиий $\varphi \in \mathscr{S}$ таких, что $\widetilde{\varphi}(t)=P_{k}(t) \widetilde{\psi}(t)$ для некоторого $k=0,1, \ldots, N$ и $\widetilde{\psi}(t) \in \mathscr{S}$. Более того,

$$
P_{k}\left(i \frac{\partial}{\partial x}\right) f(x) \in \mathbb{L}(\mathscr{S} \rightarrow \mathbb{B}), \quad k=0,1, \ldots, N
$$

В частности, существуют постоянные с и $M$ такие, что

$$
\left\|\left(P_{k}\left(i \frac{\partial}{\partial x}\right) f(x), \varphi(x)\right)\right\|_{\mathbb{B}} \leqslant c \mathscr{P}_{M}[\varphi], \quad k=0, \ldots, N, \quad \varphi \in \mathscr{S}
$$


ДокаЗАТЕльство. Сначалапокажем, что существуют постоянные $C_{1}, a_{1}$ и $N_{1}$ такие, что $\forall k=0,1, \ldots, N$ и $\forall \widetilde{\psi}(t) \in \mathscr{S}$, supp $\widetilde{\psi} \subset\{|t|<A\}, A>1$, имеет место включение $\left(\tilde{f}(t), P_{k}(t) \tilde{\psi}(t)\right) \in \mathbb{B}$ и выполнена оценка

$$
\left\|\left(\widetilde{f}(t), P_{k}(t) \widetilde{\psi}(t)\right)\right\|_{\mathbb{B}} \leqslant C_{1} A^{a_{1}} \mathscr{P}_{N_{1}}[\widetilde{\psi}] .
$$

Действительно, пусть $\operatorname{supp} \widetilde{\psi} \subset\{|t|<A\}$ и $\left|\widetilde{\omega}_{1}(t)\right| \geqslant \varepsilon$ при $|t| \leqslant \delta<A$. Согласно лемме П.3 для любой $\widetilde{\chi}(t) \in \mathscr{S}$ имеем

$$
\left\|\left(\widetilde{f}(t), \widetilde{\chi}(t) y^{b} \widetilde{\omega}_{1}(y t) \sum_{k=0}^{N} y^{k} P_{k}(t)\right)\right\|_{\mathbb{B}} \leqslant C \int(1+|x|)^{a}\left|F^{-1}[\widetilde{\chi}(t)](x)\right| d x .
$$

Полагаем здесь $\tilde{\chi}(t)=\left(1 / \widetilde{\omega}_{1}(y t)\right) \widetilde{\psi}(t)$ и считаем, что $\delta /(2 A) \leqslant y \leqslant \delta / A$. Тогда $\tilde{\chi} \in \mathscr{S}$, так как на носителе $\widetilde{\psi}$ при таком выборе $y$ справедливо $\left|\widetilde{\omega}_{1}(y t)\right| \geqslant \varepsilon$. Имеем

$$
\begin{aligned}
\left\|\left(\widetilde{f}(t), \sum_{k=0}^{N} y^{k} \widetilde{\psi}(t) P_{k}(t)\right)\right\|_{\mathbb{B}} & \leqslant \frac{C}{y^{b}} \int(1+|x|)^{a}\left|F^{-1}\left[\frac{1}{\widetilde{\omega}_{1}(y t)} \widetilde{\psi}(t)\right](x)\right| d x \\
& \leqslant \operatorname{const} A^{b} \mathscr{P}_{N_{1}}[\widetilde{\psi}]
\end{aligned}
$$

Мы учли, что $1 / y \leqslant 2 A / \delta$ и

$$
\int(1+|x|)^{a}\left|F^{-1}\left[\frac{1}{\widetilde{\omega}_{1}(y t)} \widetilde{\psi}(t)\right](x)\right| d x \leqslant C_{2} \mathscr{P}_{N_{1}}\left[\frac{1}{\widetilde{\omega}_{1}(y t)} \widetilde{\psi}(t)\right] \leqslant C_{3} \mathscr{P}_{N_{1}}[\widetilde{\psi}(t)] .
$$

Откуда имеем

$$
\sum_{k=0}^{N}\left(\tilde{f}(t), P_{k}(t) \widetilde{\psi}(t)\right) y^{k}=C_{y}, \quad\left\|C_{y}\right\|_{\mathbb{B}} \leqslant C_{4} A^{b} \mathscr{P}_{N_{1}}[\widetilde{\psi}]
$$

Положим в $(2.6) y_{i}=\delta(1+i / N) /(2 A), i=0,1, \ldots, N$. Получим систему $N+1$ уравнения с $N+1$ неизвестным. Определитель этой системы есть определитель Вандермонда

$$
W\left[y_{0}, \ldots, y_{N}\right]=\left(\frac{\delta}{2 A}\right)^{\sigma} W\left[1,1+\frac{1}{N}, \ldots, 2\right] \neq 0, \quad \sigma=\frac{N(N+1)}{2} .
$$

Решая эту систему, для любого $k=0,1, \ldots, N$ выводим соотношение (2.5).

Продолжим доказательство леммы. Возьмем "шапочку" $\Omega(t) \in \mathscr{S}$ такую, что $\operatorname{supp} \Omega \subset\{|t|<1\}$ и $\Omega(0)=1$. По уже доказанному (см. (2.5)) для любой $\widetilde{\chi} \in \mathscr{S}$, учитывая, что $\operatorname{supp} \Omega(y t) \subset\{|t|<1 / y\}$, имеем

$$
\left\|\left(\tilde{f}(t), P_{k}(t) \Omega(y t) \widetilde{\chi}(t)\right)\right\|_{\mathbb{B}} \leqslant \frac{C_{1}}{y^{a_{1}}} \mathscr{P}_{N_{1}}[\Omega(y t) \widetilde{\chi}(t)], \quad 0<y \leqslant 1 .
$$

Откуда

$$
\left\|\left(\tilde{f}(t), y^{a_{1}} P_{k}(t) \Omega(y t) \widetilde{\chi}(t)\right)\right\|_{\mathbb{B}} \leqslant C_{1} \mathscr{P}_{N_{1}}[\Omega(y t) \widetilde{\chi}(t)] \leqslant \operatorname{const} \mathscr{P}_{N_{1}}[\widetilde{\chi}(t)] .
$$

Заметим, что семейство функций $\left\{\eta_{\beta}(t)=\beta^{a_{1}} \Omega(\beta t), 0<\beta \leqslant 1\right\}$ удовлетворяет всем условиям леммы П.1. 
Действительно, оценка (П.1) очевидна, проверим оценку (П.2). Так как $\Omega(0)=1$, то сушествует $\varepsilon>0$ такое, что $|\Omega(t)| \geqslant \frac{1}{2}$ при $|t| \leqslant \varepsilon$. Поэтому

$$
\sup _{0<\beta \leqslant 1}\left|\beta^{a_{1}} \Omega(\beta t)\right| \geqslant \beta_{0}^{a_{1}}\left|\Omega\left(\beta_{0} t\right)\right|, \quad 0<\beta_{0} \leqslant 1
$$

Выбирая теперь $\beta_{0}=\varepsilon /|t|$ при $|t|>\varepsilon$ и $\beta_{0}=1$ при $|t| \leqslant \varepsilon$, получаем

$$
\beta_{0}^{a_{1}}\left|\Omega\left(\beta_{0} t\right)\right| \geqslant \begin{cases}\left(\frac{\varepsilon}{|t|}\right)^{a_{1}}\left|\Omega\left(\varepsilon \frac{t}{|t|}\right)\right| \geqslant \frac{1}{2} \frac{\varepsilon^{a_{1}}}{|t|^{a_{1}}}, & |t|>\varepsilon \\ |\Omega(t)| \geqslant \frac{1}{2}, & |t| \leqslant \varepsilon .\end{cases}
$$

Следовательно, существуют счетное множество чисел $y_{i} \in(0,1]$, числа $\lambda_{i}>0$, $\sum_{i=1}^{\infty} \lambda_{i}=1$, и семейство функций $g_{i}(t), i=0,1, \ldots$, со свойствами (П.3) такие, что

$$
\sum_{i=1}^{\infty} \lambda_{i} g_{i}(t) y_{i}^{a_{1}} \Omega\left(y_{i} t\right)=1
$$

Пусть теперь $\psi$ любая функция из $\mathscr{S}$. Для любого $k=0, \ldots, N$ получим

$$
P_{k}(t) \widetilde{\psi}(t)=\sum_{i=1}^{\infty} \lambda_{i} g_{i}(t) \widetilde{\psi}(t) y_{i}^{a_{1}} P_{k}(t) \Omega\left(y_{i} t\right)
$$

Отсюда

$$
\left(\widetilde{f}(t), P_{k}(t) \widetilde{\psi}(t)\right)=\sum_{i=1}^{\infty} \lambda_{i}\left(\widetilde{f}(t), g_{i}(t) \widetilde{\psi}(t) y_{i}^{a_{1}} P_{k}(t) \Omega\left(y_{i} t\right)\right) .
$$

Это равенство справедливо в $\mathscr{L}$ (поскольку ряд (2.8) сходится в $\mathscr{S}$ ). Покажем, что ряд в (2.9) сходится в $\mathbb{B}$. Для этого оценим каждый член этого ряда. Из (2.7) имеем

$$
\left\|\left(\tilde{f}(t), g_{i}(t) \widetilde{\psi}(t) y_{i}^{a_{1}} P_{k}(t) \Omega\left(y_{i} t\right)\right)\right\|_{\mathbb{B}} \leqslant C_{5} \mathscr{P}_{N_{1}}\left[g_{i}(t) \widetilde{\psi}(t)\right] \leqslant \operatorname{const} \mathscr{P}_{N_{2}}[\widetilde{\psi}(t)]
$$

(в последнем неравенстве мы пользовались оценками (П.3)). Так как $\sum_{i=0}^{\infty} \lambda_{i}=1$, то ряд (2.9) сходится в $\mathbb{B}$ и имеет место оценка

$$
\left\|\left(\tilde{f}(t), P_{k}(t) \widetilde{\psi}(t)\right)\right\|_{\mathbb{B}} \leqslant \operatorname{const} \mathscr{P}_{N_{2}}[\psi] .
$$

Отсюда в силу непрерывности вложения $\mathbb{B} \subset \mathscr{L}$ и вытекают соотношения (2.3) и (2.4). Лемма доказана.

Лемма 2.2. Пусть $\mathbb{B}$ - банахово и $\mathscr{L}$ - локально выпуклое пространства, причем $\mathbb{B} \subset \mathscr{L}$. Пусть $f \in \mathbb{L}(\mathscr{S} \rightarrow \mathscr{L})$ u $\omega(x) \in \mathscr{S}$ такова, что $\widetilde{\omega}$ не вырождена в нуле. Предположим еще, что функиия $L_{f}^{\omega}(x, y)$ удовлетворяет условиям а) $и$ b). Тогда для любого $0<\delta<1$ и для любой $\varphi(x) \in \mathscr{S}$ такой, что $\operatorname{supp} \widetilde{\varphi} \subset\{t:|t| \geqslant 2 \delta\}$,

$$
(f, \varphi) \in \mathbb{B}, \quad \text { причем } \quad\|(f, \varphi)\|_{\mathbb{B}} \leqslant \frac{C_{1}}{\delta^{d}} \mathscr{P}_{N_{1}}[\varphi]
$$

для некоторьх $C_{1}, N_{1} u d$, зависящих только от $\omega$ и постоянных $C, a, b$ $\boldsymbol{B}(2.2)$. 
ДокаЗАТЕЛЬСтво. Пусть $\operatorname{supp} \widetilde{\varphi} \subset\{t:|t| \geqslant 2 \delta\}$ и заданы числа $\frac{1}{2}<\lambda_{0}<$ $\lambda_{1}<\cdots<\lambda_{m}<1$. Тогда по лемме П.2 сушествует $c$ такая, что выполнена оценка (П.21). Далее положим $\widetilde{\omega}(t)=T_{\widetilde{\omega}}^{m}(t)+\gamma(t)$, где $m$ определяется условием невырожденности $\widetilde{\omega}\left(\right.$ см. (1.1)). Подберем $\delta_{1}$ таким образом, чтобы $|\gamma(t)|<c|t|^{m} / 2$ при $|t|<\delta_{1}$, что возможно, так как $\gamma(t)=O\left(|t|^{m+1}\right)$ при $|t| \rightarrow 0$. Далее будем считать, что $\delta<\delta_{1}<1$. По лемме П.3 для любой $\chi \in \mathscr{S}$ имеем

$$
\left\|\left(\widetilde{f}(t), \widetilde{\chi}(t) y^{b} \widetilde{\omega}(y t)\right)\right\|_{\mathbb{B}} \leqslant C \int(1+|x|)^{a}|\chi(x)| d x .
$$

Проверим, что семейство $\left\{y^{b} \widetilde{\omega}(y t), 0<y<1\right\}$ удовлетворяет всем требованиям леммы П.1 при $|t|>\delta$. Условие (П.1) выполняется в силу оценки

$$
\left|\frac{\partial^{|j|}}{\partial t^{j}} y^{b} \widetilde{\omega}(y t)\right| \leqslant y^{b+|j|}\left|\widetilde{\omega}^{(j)}(y t)\right| \leqslant \sup _{t}\left|\widetilde{\omega}^{(j)}(y t)\right| \leqslant C_{j} .
$$

Проверим выполнение условия (П.2) при $|t|>\delta$, имеем

$$
\sup _{0<y<1} y^{b}|\widetilde{\omega}(y t)| \geqslant y_{t}^{b}\left|\widetilde{\omega}\left(y_{t} t\right)\right|
$$

где $y_{t}$ выбирается следующим образом. По $t$ строим $\bar{e}=t /|t|$. Из (П.21), где $\varepsilon=\delta$, а $N=m$, заключаем, что сушествует такое $i_{t}, i_{t}=0,1, \ldots, m$, для которого вьполняется неравенство

$$
\left|T_{\widetilde{\omega}}^{m}\left(\delta \lambda_{i_{t}} \frac{t}{|t|}\right)\right| \geqslant c \delta^{m}
$$

Полагая теперь $y_{t}=\delta \lambda_{i_{t}} /|t|$ и продолжая оценку (2.13), имеем

$$
\begin{gathered}
\sup _{0<y<1} y^{b}|\widetilde{\omega}(y t)| \geqslant y_{t}^{b}\left|T_{\widetilde{\omega}}^{m}\left(y_{t} t\right)+\gamma\left(y_{t} t\right)\right|=\left(\delta \lambda_{i_{t}} \frac{1}{|t|}\right)^{b}\left|T_{\widetilde{\omega}}^{m}\left(\delta \lambda_{i_{t}} \frac{t}{|t|}\right)+\gamma\left(\delta \lambda_{i_{t}} \frac{t}{|t|}\right)\right| \\
\geqslant\left(\delta \lambda_{i_{t}} \frac{1}{|t|}\right)^{b}\left[c \delta^{m}-\frac{c}{2}\left(\delta \lambda_{i_{t}}\right)^{m}\right] \geqslant \frac{1}{2} \frac{\delta^{m}}{|t|^{b}} \frac{c}{2} \delta^{m} \geqslant \frac{c}{4} \frac{\delta^{b+m}}{(1+|t|)^{b}}, \quad|t|>\delta .
\end{gathered}
$$

Тем самым условие (П.2) проверено.

Согласно замечанию 2 к лемме П.1 сушествуют числа $y_{i}, \lambda_{i}, i=1,2, \ldots$, $\sum_{i} \lambda_{i}=1$, и функции $g_{i}(t) \in C^{\infty}\left(\mathbb{R}^{n}\right)$ такие, что для любого мультиндекса $j$ найдутся постоянные $A_{j}, B_{j}$ и $P_{j}$, не зависящие от $\delta$, такие, что

причем

$$
\left|g_{i}^{(j)}(t)\right| \leqslant \frac{A_{j}}{\delta^{P_{j}}}(1+|t|)^{B_{j}}
$$

$$
\sum_{i=1}^{\infty} \lambda_{i} g_{i}(t) y_{i}^{b} \widetilde{\omega}\left(y_{i} t\right)=1, \quad|t|>2 \delta .
$$

Отсюда

$$
\widetilde{\varphi}(t)=\sum_{i=1}^{\infty} \lambda_{i} \widetilde{\varphi}(t) g_{i}(t) y_{i}^{b} \widetilde{\omega}\left(y_{i} t\right), \quad t \in \mathbb{R}^{n},
$$

так как $\operatorname{supp} \widetilde{\varphi} \subset\{|t|>2 \delta\}$. Теперь, пользуясь соотношением (2.12), получаем

$$
\begin{aligned}
\|(f, \varphi)\|_{\mathbb{B}} & =\|(\widetilde{f}(t), \widetilde{\varphi}(t))\|_{\mathbb{B}} \leqslant \sum_{i=1}^{\infty} \lambda_{i}\left\|\left(\widetilde{f}(t), \widetilde{\varphi}(t) g_{i}(t) y_{i}^{b} \widetilde{\omega}\left(y_{i} t\right)\right)\right\|_{\mathbb{B}} \\
& \leqslant \sum_{i=1}^{\infty} \lambda_{i} C \int(1+|x|)^{a}\left|F^{-1}\left[\widetilde{\varphi}(t) g_{i}(t)\right](x)\right| d x \leqslant \frac{C_{1}}{\delta^{d}} \mathscr{P}_{N_{1}}[\varphi]
\end{aligned}
$$

для некоторых $C_{1}, d$ и $N_{1}$. Лемма доказана. 
СЛЕДСТВИЕ. Пусть в условиях леммы 2.1 многочлен $P(t)$ такой, что для любого вектора $\bar{e}$

$$
|\bar{e}|=1, \quad P(\lambda \bar{e}) \not \equiv 0, \quad \lambda>0 .
$$

Tогда

$$
(f(x), \varphi(x)) \in \mathbb{B} \quad \forall \varphi \in \mathscr{S}
$$

такой, что

$$
\widetilde{\varphi}(t)=P_{k}(t) \widetilde{\psi}(t), \quad|t|<\delta,
$$

$\delta>0, k=0,1, \ldots, N$ u $\widetilde{\psi}(t) \in \mathscr{S}$.

Действительно, пусть $h_{1}(t) \in C^{\infty}\left(\mathbb{R}^{n}\right)$ и $h_{2}(t)$ таковы, что

$$
h_{1}(t)+h_{2}(t)=1, \quad h_{1}(t)= \begin{cases}1, & |t|<\delta \\ 0, & |t|>2 \delta .\end{cases}
$$

Пусть теперь $\widetilde{\varphi}(t)$ удовлетворяет условию (2.16), имеем

$$
(\widetilde{f}, \widetilde{\varphi})=\left(\tilde{f}, h_{1} \widetilde{\varphi}\right)+\left(\widetilde{f}, h_{2} \widetilde{\varphi}\right)=\left(\widetilde{f}(t), h_{1}(t) P_{k}(t) \widetilde{\psi}(t)\right)+\left(\widetilde{f}(t), h_{2}(t) \widetilde{\varphi}(t)\right) .
$$

Первое слагаемое справа в равенстве (2.17) по лемме 2.1 принадлежит $\mathbb{B}$ (и выполнена соответствующая оценка), с другой стороны, $h_{2}(t) \widetilde{\varphi}(t)=0$ при $|t|<\delta$ и мы можем воспользоваться леммой 2.2 .

Лемма 2.3. Пусть $\mathbb{B}$ и $\mathscr{L}$ - банаховь пространства, причем $\mathbb{B} \subset \mathscr{L}, f \in$ $\mathbb{L}(\mathscr{S} \rightarrow \mathscr{L})$ u $\omega(x) \in \mathscr{S}$ такова, что $\widetilde{\omega}(t)$ не вырождена в нуле. Предположсим еще, что функция $L_{f}^{\omega}(x, y)$ удовлетворяет условиям а) и b).

Тогда существует число $\ell$ такое, что

$$
(f(x), \varphi(x)) \in \mathbb{B}, \quad \varphi \in \mathscr{S}, \quad T_{\widetilde{\varphi}}^{\ell}(t) \equiv 0 .
$$

Более того, для таких $\varphi$ справедлива оценка

$$
\|(f, \varphi)\|_{\mathbb{B}} \leqslant C_{2} \mathscr{P}_{N_{2}}[\varphi]
$$

при некоторых $C_{2} u N_{2}$.

ДокаЗАТЕЛьство. Пусть $\chi(\xi) \in C^{\infty}\left(\mathbb{R}^{1}\right)$ такова, что

$$
\chi(\xi)=\left\{\begin{array}{ll}
0, & \xi<1 ; \\
>0, & 1<\xi<2 ; \\
0, & \xi>2
\end{array} \quad \int_{0}^{\infty} \chi\left(\frac{1}{\xi}\right) \frac{d \xi}{\xi}=1 .\right.
$$

Ясно, что

$$
\int_{0}^{\infty} \chi\left(\frac{|t|^{2}}{\xi}\right) \frac{d \xi}{\xi}=1 \quad \forall t \in \mathbb{R}^{n} \backslash\{0\} .
$$

Покажем, что для любого $M \geqslant 0$ сушествует $m_{1}$ такое, что

$$
\int_{1 / A}^{A} \chi\left(\frac{|t|^{2}}{\xi}\right) \widetilde{\varphi}(t) \frac{d \xi}{\xi} \underset{A \rightarrow+\infty}{\longrightarrow} \int_{0}^{\infty} \chi\left(\frac{|t|^{2}}{\xi}\right) \widetilde{\varphi}(t) \frac{d \xi}{\xi}=\widetilde{\varphi}(t) \quad \text { в } \quad \mathscr{S}_{M}
$$

для любой $\tilde{\varphi} \in \mathscr{S}$ такой, что $\widetilde{\varphi}^{(j)}(0)=0,|j| \leqslant m_{1}$. 
Действительно, для любого $M$ имеем

$$
\begin{gathered}
\mathscr{P}_{M}\left[\int_{A}^{\infty} \chi\left(\frac{|t|^{2}}{\xi}\right) \widetilde{\varphi}(t) \frac{d \xi}{\xi}\right]=\max _{|\ell| \leqslant M} \sup _{t \in \mathbb{R}^{n}}(1+|t|)^{M}\left|\frac{\partial^{|\ell|}}{\partial t^{\ell}} \int_{A}^{\infty} \chi\left(\frac{|t|^{2}}{\xi}\right) \widetilde{\varphi}(t) \frac{d \xi}{\xi}\right| \\
\leqslant \max _{|\ell| \leqslant M} \sup _{t \in \mathbb{R}^{n}}(1+|t|)^{M_{1}} \sum_{i, j} C_{i j} \int_{A}^{\infty}\left|\chi^{(i)}\left(\frac{|t|^{2}}{\xi}\right)\right|\left|\widetilde{\varphi}^{(j)}(t)\right| \frac{1}{\xi^{|i|}} \frac{d \xi}{\xi} .
\end{gathered}
$$

Оценим типичный член последней суммы. Учитывая, что в области интегрирования $\operatorname{supp} \chi \subset[1,2]$, а потому $1<|t|^{2} / \xi<2$, и, следовательно, $A<\xi<|t|^{2}<2 \xi$, имеем

$$
\begin{aligned}
& \sup _{|t|^{2}>A}(1+|t|)^{M_{1}} \int_{A}^{\infty}\left|\chi^{(i)}\left(\frac{|t|^{2}}{\xi}\right)\right|\left|\widetilde{\varphi}^{(j)}(t)\right| \frac{1}{\xi|i|} \frac{d \xi}{\xi} \\
& \quad \leqslant \text { const } \sup _{|t|^{2}>A} \frac{\left|\widetilde{\varphi}^{(j)}(t)\right|(1+|t|)^{M_{2}}}{|t|^{2|i|}} \underset{A \rightarrow+\infty}{\stackrel{ }{\longrightarrow}} 0 .
\end{aligned}
$$

Здесь мы учли, что $\widetilde{\varphi}^{(j)}(t) \in \mathscr{S}$ и, следовательно, стремится к нулю быстрее любой степени $1 /|t|$. Отсюда вытекает, что

$$
\mathscr{P}_{M}\left[\int_{A}^{\infty} \chi\left(\frac{|t|^{2}}{\xi}\right) \widetilde{\varphi}(t) \frac{d \xi}{\xi}\right] \underset{A \rightarrow+\infty}{\longrightarrow} 0
$$

Оценим теперь $\mathscr{P}_{M}\left[\int_{0}^{1 / A} \chi\left(|t|^{2} / \xi\right) \widetilde{\varphi}(t) / \xi d \xi\right]$. После раскрытия производной по формуле Лейбница для типичного слагаемого в выражении для нормы $\mathscr{P}_{M}$ с учетом того, что $1<|t|^{2} / \xi<2$, а следовательно, $|t|^{2}<2 \xi<2 / A$, получим

$$
\begin{aligned}
& \sup _{|t|^{2}<2 / A}(1+|t|)^{M_{1}} \int_{0}^{1 / A}\left|\chi^{(i)}\left(\frac{|t|^{2}}{\xi}\right)\right|\left|\widetilde{\varphi}^{(j)}(t)\right| \frac{1}{\xi^{|i|}} \frac{d \xi}{\xi} \\
& \leqslant \text { const } \sup _{|t|^{2}<\frac{1}{A}} \frac{\left|\widetilde{\varphi}^{(j)}(t)\right|(1+|t|)^{M_{1}}}{|t|^{2|i|}} \underset{A \rightarrow+\infty}{\longrightarrow} 0
\end{aligned}
$$

если $\widetilde{\varphi}(t)$ имеет достаточно высокий порядок нуля в начале координат. Предполагая это, получаем, что

$$
\mathscr{P}_{M}\left[\int_{0}^{1 / A} \chi\left(\frac{|t|^{2}}{\xi}\right) \widetilde{\varphi}(t) \frac{d \xi}{\xi}\right] \underset{A \rightarrow+\infty}{\longrightarrow} 0
$$

Отсюда и из (2.21) вытекает (2.20).

Продолжим доказательство леммы. Заметим, что так как $f \in \mathbb{L}(\mathscr{S} \rightarrow \mathscr{L})$ и $\mathscr{L}$ банахово, то функционал $f$ имеет конечный порядок, скажем, $M_{0}$, т.е.

$$
\|(f(x), \varphi(x))\|_{\mathscr{L}}=\|(\tilde{f}(t), \widetilde{\varphi}(t))\|_{\mathscr{L}} \leqslant \operatorname{const} \mathscr{P}_{M_{0}}[\widetilde{\varphi}] .
$$

Считая $M>M_{0}$, для любой $\widetilde{\varphi} \in \mathscr{S}$, у которой порядок нуля в начале координат достаточно велик, используя (2.20), имеем

$$
\begin{aligned}
(\tilde{f}(t), \tilde{\varphi}(t)) & =\left(\widetilde{f}(t), \int_{0}^{\infty} \chi\left(\frac{|t|^{2}}{\xi}\right) \widetilde{\varphi}(t) \frac{d \xi}{\xi}\right)=\left(\tilde{f}(t), \lim _{A \rightarrow+\infty} \int_{1 / A}^{A} \chi\left(\frac{|t|^{2}}{\xi}\right) \widetilde{\varphi}(t) \frac{d \xi}{\xi}\right) \\
& =\lim _{A \rightarrow+\infty}\left(\tilde{f}(t), \int_{1 / A}^{A} \chi\left(\frac{|t|^{2}}{\xi}\right) \widetilde{\varphi}(t) \frac{d \xi}{\xi}\right),
\end{aligned}
$$


где последний предел понимается как предел в $\mathscr{L}$. Подынтегральная функция в последнем равенстве (2.22) непрерывна по параметру $\xi$ в $\mathscr{S}$, и стандартными рассуждениями с суммами Римана можно показать, что интеграл по параметру можно вынести за знак функционала. Продолжая равества (2.22), имеем

$$
(\widetilde{f}(t), \widetilde{\varphi}(t))=\lim _{A \rightarrow+\infty} \int_{1 / A}^{A}\left(\widetilde{f}(t), \chi\left(\frac{|t|^{2}}{\xi}\right) \widetilde{\varphi}(t)\right) \frac{d \xi}{\xi},
$$

где интеграл понимается как интеграл $\Phi$ реше в $\mathscr{L}$.

Согласно лемме 2.2 функция $\left(\widetilde{f}(t), \chi\left(|t|^{2} / \xi\right) \widetilde{\varphi}(t)\right) \in \mathbb{B}$, так как носитель функции $\chi\left(|t|^{2} / \xi\right) \widetilde{\varphi}(t)$ лежит в шаровом слое $1 / A<|t|^{2}<2 A$. Интеграл в (2.23), понимаемый как интеграл $\Phi$ реше в $\mathscr{L}$, можно считать интегралом $\Phi$ реше в $\mathbb{B}$, поскольку подьнтегральная функция непрерьвна в $\mathbb{B}$. Покажем, что он не только принадлежит $\mathbb{B}$, но и при $A \rightarrow+\infty$ сходится в $\mathbb{B}$, если порядок нуля у $\widetilde{\varphi}$ в начале координат достаточно высок. Для этого достаточно показать, что норма подьптегральной функции в $\mathbb{B}$ интегрируема на $(0,+\infty)$. При $\xi>1$ из леммы 2.2 имеем

$$
\frac{1}{\xi}\left\|\left(\tilde{f}(t), \chi\left(\frac{|t|^{2}}{\xi}\right) \widetilde{\varphi}(t)\right)\right\|_{\mathbb{B}} \leqslant \operatorname{const} \mathscr{P}_{N_{1}}\left[\chi\left(\frac{|t|^{2}}{\xi}\right) \widetilde{\varphi}(t)\right] .
$$

Последнее выражение убывает быстрее любой степени $1 / \xi$ при $\xi \rightarrow+\infty$, так как $\operatorname{supp} \chi\left(|t|^{2} / \xi\right)$ лежит на отрезке $[\sqrt{\xi}, \sqrt{2 \xi}]$.

Далее, при $\xi<1$ по той же лемме имеем

$$
\begin{aligned}
& \frac{1}{\xi}\left\|\left(\tilde{f}(t), \chi\left(\frac{|t|^{2}}{\xi}\right) \widetilde{\varphi}(t)\right)\right\|_{\mathbb{B}} \leqslant \frac{C_{1}}{\xi^{d / 2+1}} \mathscr{P}_{N_{1}}\left[\chi\left(\frac{|t|^{2}}{\xi}\right) \widetilde{\varphi}(t)\right] \\
& \quad=\frac{C_{1}}{\xi^{d / 2+1}} \max _{|j| \leqslant N_{1}} \sup _{\sqrt{\xi}<|t|<\sqrt{2 \xi}}(1+|t|)^{N_{1}}\left|\frac{\partial^{|j|}}{\partial t^{j}} \chi\left(\frac{|t|^{2}}{\xi}\right) \widetilde{\varphi}(t)\right| .
\end{aligned}
$$

Ясно, что это выражение ограничено при $\xi \rightarrow+0$, если только $\widetilde{\varphi}(t)$ имеет в начале координат нуль достаточно высокого поря дка. Тем самым норма в $\mathbb{B}$ подынтегральной функции в (2.24) действительно интегрируема. Следовательно, $(f, \varphi) \in \mathbb{B}$ для таких $\varphi$. Оценка (2.19) легко следует из оценок (2.24) и (2.25). Лемма доказана.

Лемма 2.4. Пусть $\mathbb{B}$ и $\mathscr{L}$ - банаховы пространства, $\mathbb{B} \subset \mathscr{L}, f \in \mathbb{L}(\mathscr{S} \rightarrow \mathscr{L})$ $u \omega(x) \in \mathscr{S}$, причем $\widetilde{\omega}(t)$ не вырожсдена в нуле, а ее тейлоровское разложение имеет вид:

$$
T_{\widetilde{\omega}}^{N}(t)=\sum_{k=0}^{N} P_{k}(t), \quad N=0,1, \ldots,
$$

где $P_{k}(t)$ - однородный многочлен степени $k$ (либо тождественный нуль). Предполохим еще, что функиия $L_{f}^{\omega}(x, y)$ удовлетворяет условиям а) $u$ b). Тогда для любой $\varphi \in \mathscr{S}$ такой, что $\widetilde{\varphi}(t)=P_{k}(t) \widetilde{\psi}(t)$, где $\widetilde{\psi}(t) \in \mathscr{S}$, имеем

$$
(f, \varphi) \in \mathbb{B}, \quad\|(\tilde{f}, \widetilde{\varphi})\|_{\mathbb{B}} \leqslant C_{1} \mathscr{P}_{N}[\widetilde{\varphi}]
$$

для некоторых $C_{1}$ и N. Другими словами,

$$
P_{k}\left(i \frac{\partial}{\partial x}\right) f \in \mathbb{L}(\mathscr{S} \rightarrow \mathbb{B}), \quad k=0,1, \ldots
$$


ДоказАтЕльство. Сначала заметим, что согласно лемме 2.3 существует $\ell$ такое, что выполнены соотношения (2.18) и (2.19). Без ограничения общности можно считать, что $\ell>m$, где $m$ берется из условия невырожденности (см. (1.1)). Пусть $\omega_{1} \in \mathscr{S}, \widetilde{\omega}_{1}(0) \neq 0$ и $\omega_{2}(x)$ такова, что $\widetilde{\omega}_{2}(t)=T_{\widetilde{\omega}}^{\ell}(t) \widetilde{\omega}_{1}(t)$. Имеем

$$
\begin{aligned}
L_{f}^{\omega_{2}}(x, y) & =\left(\widetilde{f}(t), T_{\widetilde{\omega}(t)}^{\ell}(y t) \widetilde{\omega}_{1}(y t) e^{i x t}\right) \\
& =\left(\widetilde{f}(t), \widetilde{\omega}(y t) \widetilde{\omega}_{1}(y t) e^{i x t}\right)-\left(\widetilde{f}(t),\left(\widetilde{\omega}(y t)-T_{\widetilde{\omega}(y t)}^{\ell}(t)\right) \widetilde{\omega}_{1}(y t) e^{i x t}\right) .
\end{aligned}
$$

Мы учли, что $T_{\widetilde{\omega}(y t)}^{\ell}(t)=T_{\widetilde{\omega}(t)}^{\ell}(y t)$. Согласно лемме П.3 (см. также замечание к ней) первое слагаемое справа в (2.28) принадлежит $\mathbb{B}$ и

$$
\begin{aligned}
\left\|\left(\tilde{f}(t), \widetilde{\omega}(y t) \widetilde{\omega}_{1}(y t) e^{i x t}\right)\right\|_{\mathbb{B}} & \leqslant \frac{\text { const }}{y^{b}} \mathscr{P}_{N}\left[\widetilde{\omega}_{1}(y t) e^{i x t}\right] \\
& \leqslant \frac{\text { const }}{y^{b_{1}}}(1+|x|)^{N} \mathscr{P}_{N}\left[\widetilde{\omega}_{1}\right],
\end{aligned}
$$

где $b_{1}$ и $N$ - некоторые постоянные. Из той же леммы П.3 следует, что левая часть (2.28) непрерьвна по $x$ со значениями в $\mathbb{B}$. Второе слагаемое справа в (2.28) согласно лемме 2.3 также принадлежит $\mathbb{B}$ и

$$
\begin{aligned}
\left\|\left(\widetilde{f}(t),\left(\widetilde{\omega}(y t)-T_{\widetilde{\omega}(y t)}^{\ell}(t)\right) \widetilde{\omega}_{1}(t) e^{i x t}\right)\right\|_{\mathbb{B}} & \leqslant C_{2} \mathscr{P}_{N_{1}}\left[\left(\widetilde{\omega}(y t)-T_{\widetilde{\omega}(y t)}^{\ell}(t)\right) \widetilde{\omega}_{1}(t) e^{i x t}\right] \\
& \leqslant C_{3} \frac{(1+|x|)^{a_{2}}}{y^{b_{2}}} \mathscr{P}_{N_{3}}\left[\widetilde{\omega}_{1}(t)\right],
\end{aligned}
$$

где $a_{2}, C_{3}, b_{2}$ и $N_{3}$ - некоторые постоянные. Из той же леммы 2.3 следует, что левая часть (2.28) непрерывна по $x$ со значениями в $\mathbb{B}$. Следовательно, $L_{f}^{\omega_{2}}(x, y)-$ непрерывная функция по $x$ со значениями в $\mathbb{B}$ и справедлива оценка

$$
\left\|L_{f}^{\omega_{2}}(x, y)\right\|_{\mathbb{B}} \leqslant \mathrm{C}_{4} \frac{(1+|x|)^{a_{3}}}{y^{b_{3}}},
$$

для некоторых $C_{4}, a_{3}$ и $b_{3}$. Отсюда из леммы 2.1 и будут следовать соотношения (2.26) и (2.27). Лемма доказана.

Tеорема 2.1. Пусть $\mathbb{B}, \mathscr{L}$ - банаховы пространства, $\mathbb{B} \subset \mathscr{L}, f \in \mathbb{L}(\mathscr{S} \rightarrow \mathscr{L})$ $u \omega(x) \in \mathscr{S}$, причем $\widetilde{\omega}(t)$ не вырождена в нуле. Пусть, кроме того, стандартное усреднение $L_{f}^{\omega}(x, y)$ удовлетворяет условиям а) $и$ b). Обозначим через $P_{k}(t), k=0,1, \ldots$, однородные многочлены степени $k$ такие, что

$$
T_{\widetilde{\omega}}^{N}(t)=\sum_{k=0}^{N} P_{k}(t), \quad N=0,1, \ldots
$$

Тогда существует число $\ell$ такое, что

$$
f \in \mathbb{L}\left(\widetilde{\mathscr{S}}_{\left[\ell ; P_{0}, P_{1}, \ldots, P_{\ell}\right]} \rightarrow \mathbb{B}\right) .
$$

Другими словами, для любой $\varphi \in \widetilde{\mathscr{S}}_{\left[\ell ; P_{0}, \ldots, P_{\ell}\right]}$

$$
(f, \varphi) \in \mathbb{B}, \quad\|(f, \varphi)\|_{\mathbb{B}}=\|(\tilde{f}, \widetilde{\varphi})\|_{\mathbb{B}} \leqslant C_{1} \mathscr{P}_{N}[\widetilde{\varphi}]
$$

для некоторых $C_{1}$ u $N$. 
ДОКАЗАТЕЛЬСТво. Пусть

$$
\eta(t) \in \mathscr{S}, \quad \eta(t)= \begin{cases}1, & |t|<1 \\ 0, & |t|>2\end{cases}
$$

Заметим, что по лемме 2.3 сушествует $\ell$ такое, что выполнены соотношения (2.18) и (2.19). При этом без нарушения обшности можно считать, что $\ell>m$, где $m$ берется из условия невырожденности (см. (1.1)). Пусть теперь $\varphi \in \widetilde{\mathscr{S}}_{\left[\ell ; P_{0}, P_{1}, \ldots, P_{\ell}\right]}$, имеем

$$
\begin{aligned}
(f(x), \varphi(x)) & =(\widetilde{f}(t), \widetilde{\varphi}(t))=(\widetilde{f}(t), \eta(t) \widetilde{\varphi}(t))+(\widetilde{f}(t),(1-\eta(t)) \widetilde{\varphi}(t)) \\
& =\left(\widetilde{f}(t), \eta(t)\left(\widetilde{\varphi}(t)-T_{\widetilde{\varphi}}^{\ell}(t)\right)\right)+(\widetilde{f}(t),(1-\eta(t)) \widetilde{\varphi}(t))+\left(\widetilde{f}(t), \eta(t) T_{\tilde{\varphi}}^{\ell}(t)\right) .
\end{aligned}
$$

Первое и второе слагаемые справа по леммам 2.3 и 2.2 соответственно принадлежат $\mathbb{B}$ и оцениваются должным образом. Рассмотрим третье слагаемое. По условию

$$
T_{\widetilde{\varphi}}^{\ell}(t)=\sum_{k=0}^{\ell} P_{k}(t) Q_{k}(t),
$$

где без ограничения обшности можно считать, что $Q_{k}(t)$ являются многочленами степени не выше $\ell-k$ и выполняется оценка (1.3). Имеем

$$
\left(\widetilde{f}(t), \eta(t) T_{\widetilde{\varphi}}^{\ell}(t)\right)=\sum_{k=0}^{\ell}\left(\widetilde{f}(t), P_{k}(t) Q_{k}(t) \eta(t)\right) .
$$

Применяя теперь лемму 2.4 в которой $\widetilde{\psi}(t)=Q_{k}(t) \eta(t), k=0, \ldots, \ell$, и пользуясь оценкой (1.3), завершаем доказательство теоремы.

ЗАмечАниЕ. Теорема 2.1 как, впрочем, и леммы 2.1-2.4, останутся справедливьми, если условие а) в них заменить на следующее условие $\left.\mathrm{a}_{1}\right)$.

Пусть $f \in \mathbb{L}(\mathscr{S} \rightarrow \mathscr{L}), \mathbb{B}$ - банахово пространство и $\left\{\mathbb{B}_{\varepsilon}, \varepsilon>0\right\}-$ семейство банаховых пространств такое, что $\mathbb{B} \subset \mathbb{B}_{\varepsilon} \subset \mathscr{L}$, причем

$$
\mathbb{B}=\left\{\eta \in \bigcap_{\varepsilon>0} \mathbb{B}_{\varepsilon}: \sup _{\varepsilon>0}\|\eta\|_{\mathbb{B}_{\varepsilon}}<\infty\right\}, \quad\|\eta\|_{\mathbb{B}_{\varepsilon}} \nearrow\|\eta\|_{\mathbb{B}}, \quad \varepsilon \rightarrow+0
$$

Будем говорить, что для некоторого ядра $\omega \in \mathscr{S}$ усреднение $L_{f}^{\omega}(x, y)$ удовлетворяет условию $\left.\mathrm{a}_{1}\right)$, если при каждом $y \in(0,1]$ функция $L_{f}^{\omega}(x, y) \in \mathbb{B}$ при почти всех $x \in \mathbb{R}^{n}$ и измерима как функция со значениями в $\mathbb{B}_{\varepsilon}, \varepsilon>0$.

Действительно, просматривая доказательство лемм 2.1-2.4, нетрудно видеть, что постоянные $C_{1}$ и $N$ в (2.32) зависят только от $\omega$ и постоянных $C, a$ и $b$ из условия b), а число $\ell$ зависит еше и от порядка сингулярности отображения $f: \mathscr{S} \rightarrow \mathscr{L}$. В частности, они не зависят от "конкретного вида" банахова пространства $\mathbb{B}$. Поэтому, пользуясь теоремой 2.1 для пространства $\mathbb{B}_{\varepsilon}$, получаем $(f, \varphi) \in \mathbb{B}_{\varepsilon}$, причем

$$
\|(f, \varphi)\|_{\mathbb{B}_{\varepsilon}} \leqslant C_{1} \mathscr{P}_{N}[\varphi] \quad \forall \varphi \in \widetilde{\mathscr{S}}_{\left[\ell, P_{0}, \ldots, P_{\ell}\right]},
$$

где $C_{1}$ и $N$ не зависят от $\varepsilon$. Отсюда и следует наше утверждение.

Отметим, что конкретные применения теоремы 2.1 затруднены тем, что число $\ell$, фигурируюшее в этом утверждении, вообще говоря, зависит от обобшенной функции $f$ (точнее, от ее порядка сингулярности в $\mathscr{L}$ ) и, как правило, заранее не известно. Поясним это следующим примером. 
ПРИМЕР 2.1. Пусть $n=2$ и $\widetilde{\omega}\left(t_{1}, t_{2}\right)=\left(t_{1}^{2}+t_{2}^{2}\right) \widetilde{\omega}_{1}\left(t_{1}, t_{2}\right)$, где $\widetilde{\omega}_{1}(0,0) \neq 0$. Банахово пространство $\mathbb{B}=\{0\}$, а $\mathscr{L}=\mathbb{C}$. Пусть $f_{k}\left(x_{1}, x_{2}\right)-$ гармонический многочлен степени $k$ (например, можно положить $f_{k}\left(x_{1}, x_{2}\right)=\operatorname{Re} P_{k}\left(x_{1}+i x_{2}\right)$, где $P_{k}(z)$ - многочлен степени $\left.k\right)$. Имеем

$$
\begin{aligned}
L_{f_{k}}^{\omega}(x, y) & =\left(f_{k}(\xi), \frac{1}{y^{2}} \omega\left(\frac{x-\xi}{y}\right)\right)=\left(f_{k}(\xi),-\Delta_{\xi} \omega_{1}\left(\frac{x-\xi}{y}\right)\right) \\
& =\left(\Delta f_{k}(\xi), \omega_{1}\left(\frac{x-\xi}{y}\right)\right)=0 .
\end{aligned}
$$

В последнем равенстве (2.33) учтено, что $f_{k}$ - гармоническая функция. Таким образом, все условия леммы 2.3 выполнены, однако

$$
\left(f_{k}(x), \varphi(x)\right)=\left(\tilde{f}_{k}(t), \widetilde{\varphi}(t)\right)=\sum_{|j|=0}^{k}\left(C_{j} \delta^{(j)}(t), \widetilde{\varphi}(t)\right)
$$

Поскольку хотя бы один из старших коэффициентов $C_{j},|j|=k$, отличен от нуля, скажем $C_{j_{0}}$, то, взяв функцию $\widetilde{\varphi}(t) \in \mathscr{S}$, у которой все производные до порядка $k$ в нуле равны нулю кроме $\widetilde{\varphi}^{\left(j_{0}\right)}(0) \neq 0$, получим $\left(f_{k}, \varphi\right)=(-1)^{k} C_{j_{0}} \widetilde{\varphi}^{\left(j_{0}\right)}(0) \neq 0$. Следовательно, $\ell$ в лемме 2.3 должно быть больше или равно $k$, т.е. может быть как угодно велико.

Однако в некоторых случаях эту трудность удается обойти. Один из примеров такой ситуации приводится в следующей теореме 2.2. Другие примеры будут рассмотрены далее в следуюшем параграфе (см. следствие к теоремам 3.2 и 3.3).

ТЕОРема 2.2. Пусть выполнены все условия теоремы 2.1 и, кроме того, существуют числа $M$ и $N$ такие, что

$$
\mathbb{P}^{M} \subset \mathbb{P}\left[P_{0}, \ldots, P_{N}\right]
$$

Тогда в теореме 2.1 можно считать, что $\ell=M$.

ДокАЗАТЕЛЬСтво. Пусть $T_{\widetilde{\varphi}}^{M}(t)=\sum_{k=0}^{N} P_{k}(t) Q_{k}(t)$, где $Q_{k}$ - многочлены, и $\ell$ - число, фигурирующее в теореме 2.1 (без ограничения общности можно считать $\ell \geqslant M)$. Тогда

$$
T_{\widetilde{\varphi}}^{\ell}(t)=\sum_{k=0}^{M} P_{k}(t) Q_{k}(t)+\widehat{P}(t)
$$

где $\widehat{P} \in \mathbb{P}^{M}$ (т.е. все младшие члены его до порядка $M$ включительно равны нулю). По условию $\widehat{P}(t)=\sum_{k=0}^{N} P_{k}(t) \widehat{Q}_{k}(t)$, где $\widehat{Q}_{k}(t)$ - некоторые многочлены. Таким образом,

$$
T_{\widetilde{\varphi}}^{\ell}(t)=\sum_{k=0}^{N} P_{k}(t)\left(Q_{k}(t)+\widehat{Q}_{k}(t)\right)
$$

Следовательно, $T_{\widetilde{\varphi}}^{\ell}(t) \in \mathbb{P}\left[P_{0}, \ldots, P_{N}\right]$. Пользуясь теперь теоремой 2.1 , завершаем доказательство. 
Пример 2.2. Пусть выполнены все условия теоремы 2.1 с функцией $\omega \in \mathscr{S}$ такой, что $\widetilde{\omega}\left(t_{1}, t_{2}\right)=t_{1}+t_{2}^{2}+o\left(|t|^{2}\right)$ при $t \rightarrow 0\left(t \in \mathbb{R}^{2}\right)$. Легко видеть, что вьполнены все условия теоремы 2.2 с $M=1$. Тем самьм соотношение (2.32) будет вьполняться для всех $\varphi \in \widetilde{\mathscr{S}}_{\left[1 ; 0, t_{1}\right]}$ или согласно лемме П.5 для всех $\varphi \in \mathscr{S}$, для которых

$$
\int \varphi(x) d x=0, \quad \int x_{2} \varphi(x) d x=0
$$

\section{§3. Абелевы и тауберовы теоремы}

В этом параграфе мы доказываем абелевы и тауберовы теоремы для стандартного усреднения обобщенных функций медленного роста со значениями в банаховом пространстве и связанные с их квазиасимптотикой. Следующая теорема, по сути, является теоремой абелева типа и вытекает из общих теорем функционального анализа.

ТеОРема 3.1. Пусть $\mathbb{B}$ - банахово пространство, $f \in \mathbb{L}(\mathscr{S} \rightarrow \mathbb{B}), \rho(k)$ автомодельная функиия, $M$ - неотрицательное целое число, $\left\{P_{k}(t), k=\right.$ $0,1, \ldots, m\}$ - семейство многочленов, причем $P_{k}(t)$ - либо однородный многочлен степени $k$, либо тождественный нуль. Предположим, что

$$
\sup _{k \geqslant 1} \frac{1}{\rho(k)}\left\|\left(f\left(\frac{\xi}{k}\right), \varphi(\xi)\right)\right\|_{\mathbb{B}}<\infty \quad \forall \varphi \in \widetilde{\mathscr{S}}_{\left[M ; P_{0}, P_{1}, \ldots, P_{m}\right]} .
$$

Тогда существуют постоянные $C$ и $N$ такие, что

$$
\sup _{k \geqslant 1} \frac{1}{\rho(k)}\left\|\left(f\left(\frac{\xi}{k}\right), \varphi(\xi)\right)\right\|_{\mathbb{B}} \leqslant C \mathscr{P}_{N}[\varphi] \quad \forall \varphi \in \widetilde{\mathscr{S}}_{\left[M ; P_{0}, P_{1}, \ldots, P_{m}\right]} .
$$

ДокАЗАТЕЛЬСтво. Рассмотрим семейство полунорм на $\mathscr{S}$

$$
Q_{\varkappa}[\varphi]=\sup _{1 \leqslant k \leqslant \varkappa} \frac{1}{\rho(k)}\left\|\left(f\left(\frac{\xi}{k}\right), \varphi(\xi)\right)\right\|_{\mathbb{B}}, \quad \varkappa>1 .
$$

Они непрерывны на $\mathscr{S}$, монотонно по $\varkappa$ возрастают и по условию

$$
Q[\varphi]=\sup _{\varkappa} Q_{\varkappa}[\varphi]<\infty \quad \forall \varphi \in \widetilde{\mathscr{S}}_{\left[M ; P_{0}, \ldots, P_{m}\right]}
$$

Таким образом, функционал $Q[\varphi]$ полунепрерьвен снизу на $\mathscr{S}$ и ограничен на $\widetilde{\mathscr{S}}_{\left[M ; P_{0}, \ldots, P_{m}\right]}$. Так как $\widetilde{\mathscr{S}}_{\left[M ; P_{0}, \ldots, P_{m}\right]}$ - замкнутое подпространство пространства фреше (см. $\S 1)$, оно само - пространство Фреше, в частности, бочечно. А потому $Q[\varphi]$ - непрерывная полунорма на $\widetilde{\mathscr{S}}_{\left[M ; P_{0}, \ldots, m\right]}$, что и доказывает $(3.2)$. Теорема доказана.

СлЕДСТВИЕ. Пусть выполнены условия теоремы 3.1. Тогда существуют постоянные $A, b, N$ и а такие, что

$$
\sup _{k \geqslant 1} \frac{1}{\rho(k)}\left\|L_{f}^{\varphi}\left(\frac{x}{k}, \frac{y}{k}\right)\right\|_{\mathbb{B}} \leqslant \frac{A(1+|x|)^{a}}{y^{b}} \mathscr{P}_{N}[\varphi], \quad x \in \mathbb{R}^{n}, \quad y \in(0,1)
$$

для всех $\varphi \in \widetilde{\mathscr{S}}_{\left[M ; P_{0}, \ldots, P_{m}\right]}$. 
ДоказАтельство. Заметим сначала, что если $\varphi \in \widetilde{\mathscr{S}}_{\left[M ; P_{0}, P_{1}, \ldots, P_{m}\right]}$, т.е. $\widetilde{\varphi}(t) \in$ $\mathscr{S}_{\left[M ; P_{0}, P_{1}, \ldots, P_{m}\right]}$, то также $\left(1 / y^{n}\right) \varphi((x-\xi) / y) \in \widetilde{\mathscr{S}}_{\left[M ; P_{0}, P_{1}, \ldots, P_{m}\right]}$, так как

$$
F_{\xi}\left[\frac{1}{y^{n}} \varphi\left(\frac{x-\xi}{y}\right)\right]=e^{i x t} \widetilde{\varphi}(y t) \in \mathscr{S}_{\left[M ; P_{0}, \ldots, P_{m}\right]} .
$$

Пользуясь теперь оценкой (3.2), в которую вместо $\varphi$ подставлена функция $\left(1 / y^{n}\right) \varphi((x-\xi) / y)$, получаем

$$
\begin{aligned}
\sup _{k \geqslant 1} \frac{1}{\rho(k)}\left\|\left(f\left(\frac{\xi}{k}\right), \frac{1}{y^{n}} \varphi\left(\frac{x-\xi}{y}\right)\right)\right\|_{\mathbb{B}} & \leqslant C \mathscr{P}_{N}\left[\frac{1}{y^{n}} \varphi\left(\frac{x-\xi}{y}\right)\right] \\
& \leqslant A \frac{(1+|x|)^{N+1}}{y^{n+N}} \mathscr{P}_{N}[\varphi]
\end{aligned}
$$

что и требовалось. Следствие доказано.

ТЕОРема 3.1'. Пусть выполнены условия теоремы 3.1 и $f$ обладает квазиасимптотикой в нуле относительно $\rho(k)$ на $\widetilde{\mathscr{S}}_{\left[M ; P_{0}, P_{1}, \ldots, P_{m}\right]}$, m.е. существyem

$$
\lim _{k \rightarrow+\infty} \frac{1}{\rho(k)}\left(f\left(\frac{\xi}{k}\right), \varphi(\xi)\right)=C_{\varphi} \quad \forall \varphi \in \widetilde{\mathscr{S}}_{\left[M ; P_{0}, P_{1}, \ldots, P_{m}\right]}
$$

Тогда существует

$$
\lim _{k \rightarrow+\infty} \frac{1}{\rho(k)} L_{f}^{\varphi}\left(\frac{x}{k}, \frac{y}{k}\right)=C_{\varphi}(x, y) \quad \forall \varphi \in \widetilde{\mathscr{S}}_{\left[M ; P_{0}, P_{1}, \ldots, P_{m}\right]}
$$

и выполнена оценка (3.3).

ДоКАЗАТЕЛЬСтво тривиально следует из теоремы 3.1 и формулы

$$
L_{f}^{\varphi}\left(\frac{x}{k}, \frac{y}{k}\right)=\left(f\left(\frac{\xi}{k}\right), \frac{1}{y^{n}} \varphi\left(\frac{x-\xi}{y}\right)\right) .
$$

ЗАмечАниЕ. Теорема 3.1 , следствие к ней и теорема $3.1^{\prime}$ останутся справедливыми, если вместо квазиасимптотической ограниченности (квазиасимптотики) в нуле рассматривать квазиасимптотическую ограниченность (квазиасимптотику) на беконечности. Другими словами, если всюду в условиях вместо $f(\xi / k)$ и $L_{f}^{\varphi}(x / k, y / k)$ подставить $f(k \xi)$ и $L_{f}^{\varphi}(k x, k y)$ соответственно.

Следуюшие теоремы можно интерпретировать как теоремы тауберова типа. Их формулировке и доказательству предпошлем несколько лемм.

Лемма 3.1. Пусть $\mathbb{B}$ - банахово пространство, $\rho(k)$ - автомодельная функиия, $f \in \mathbb{L}(\mathscr{S} \rightarrow \mathbb{B})$ и $\omega(x) \in \mathscr{S}$, причем $\widetilde{\omega}(t)$ не вырождена в нуле, а ее тейлоровское разлохсение в начале координат имеет вид:

$$
T_{\widetilde{\omega}}^{N}(t)=\sum_{q=0}^{N} P_{q}(t), \quad q=0,1, \ldots, N, \quad N=0,1, \ldots,
$$


где $P_{q}(t)$ - однородный многочлен степени $q$ (либо тождественный нуль). Пусть еще существуют постоянные $A$ и $b$ такие, что

$$
\sup _{k>1} \frac{1}{\rho(k)}\left\|L_{f}^{\omega}\left(\frac{x}{k}, \frac{y}{k}\right)\right\|_{\mathbb{B}} \leqslant \frac{A}{y^{b}}, \quad|x|^{2}+y^{2}=1, \quad y>0
$$

Тогда существует $\ell$ такое, что $f$ квазиасимптотически ограничено в нуле относительно $\rho(k)$ на мнохестве $\widetilde{\mathscr{S}}_{\left[\ell ; P_{0}, P_{1}, \ldots, P_{\ell}\right]}$, m.е.

$$
\sup _{k>1} \frac{1}{\rho(k)}\left\|\left(f\left(\frac{\xi}{k}\right), \varphi(\xi)\right)\right\|_{\mathbb{B}}<\infty \quad \forall \varphi \in \widetilde{\mathscr{S}}_{\left[\ell ; P_{0}, P_{1}, \ldots, P_{\ell}\right]} .
$$

ДокаЗАТЕльство. В силу конечности порядка $f$ сушествуют $Q$ и $C_{1}$ такие, что $\|(f, \varphi)\|_{\mathbb{B}} \leqslant C_{1} \mathscr{P}_{Q}[\varphi]$, поэтому

$$
\left\|\left(f\left(\frac{\xi}{k}\right), \varphi(\xi)\right)\right\|_{\mathbb{B}}=k^{n}\|(f(\xi), \varphi(k \xi))\|_{\mathbb{B}} \leqslant C_{2} k^{Q_{1}} \mathscr{P}_{Q}[\varphi], \quad k>1,
$$

для некоторых $C_{2}$ и $Q_{1}$. Без ограничения общности можно считать, что $Q_{1}>\alpha$. Введем банаховы пространства

$$
\begin{aligned}
& \mathscr{L}=\left\{\gamma(k) \in C([1, \infty) \rightarrow \mathbb{B}):\|\gamma(k)\|_{\mathscr{L}}=\sup _{k>1} \frac{1}{k^{Q_{1}}}\|\gamma(k)\|_{\mathbb{B}}<\infty\right\}, \\
& G=\left\{\gamma(k) \in C([1, \infty) \rightarrow \mathbb{B}):\|\gamma(k)\|_{G}=\sup _{k>1} \frac{1}{\rho(k)}\|\gamma(k)\|_{\mathbb{B}}<\infty\right\} .
\end{aligned}
$$

Очевидно, что $G \subset \mathscr{L}$. Из (3.9) следует, что отображение $g: \varphi \mapsto(f(\xi / k)$, $\varphi(\xi))=\gamma_{\varphi}(k)$ непрерывно из $\mathscr{S}$ в $\mathscr{L}$. Поэтому обобшенная функция $g(\xi)$, задаваемая формулой

$$
(g(\xi), \varphi(\xi))=\left(f\left(\frac{\xi}{k}\right), \varphi(\xi)\right)
$$

принадлежит $\mathbb{L}(\mathscr{S} \rightarrow \mathscr{L})$. Заметим, что

$$
L_{g}^{\omega}(x, y)=\left(g(\xi), \frac{1}{y^{n}} \omega\left(\frac{x-\xi}{y}\right)\right)=\left(f\left(\frac{\xi}{k}\right), \frac{1}{y^{n}} \omega\left(\frac{x-\xi}{y}\right)\right)=L_{f}^{\omega}\left(\frac{x}{k}, \frac{y}{k}\right) .
$$

Из условия (3.7) и леммы П.4 следует, что существуют постоянные $A_{1}, b_{1}$ и $a_{1}$ такие, что

$$
\left\|L_{g}^{\omega}(x, y)\right\|_{G}=\sup _{k \geqslant 1} \frac{1}{\rho(k)}\left\|L_{f}^{\omega}\left(\frac{x}{k}, \frac{y}{k}\right)\right\|_{\mathbb{B}} \leqslant \frac{A_{1}}{y^{b_{1}}}(1+|x|)^{a_{1}}, \quad x \in \mathbb{R}^{n}, \quad y \in(0,1) .
$$

Тем самьм для $L_{g}^{\omega}(x, y)$ выполнено условие b) теоремы 2.1, в котором надо банахово пространство $\mathbb{B}$ заменить на $G$ и $f$ на $g$. Докажем, что выполнено и условие $\left.\mathrm{a}_{1}\right)$ (см. замечание к теореме 2.1). Для этого при любом $\varepsilon>0$ введем банахово пространство

$$
G_{\varepsilon}=\left\{\gamma(k) \in C([1, \infty) \rightarrow \mathbb{B}):\|\gamma(k)\|_{G_{\varepsilon}}=\sup _{k>1} \frac{1}{k^{\varepsilon} \rho(k)}\|\gamma(k)\|_{\mathbb{B}}<\infty\right\} .
$$


Ясно, что

$$
G=\left\{\gamma \in \bigcap_{\varepsilon>0} G_{\varepsilon}: \sup _{\varepsilon>0}\|\gamma\|_{G_{\varepsilon}}<\infty\right\}, \quad\|\gamma\|_{G_{\varepsilon}} \nearrow\|\gamma\|_{G}, \quad \varepsilon \rightarrow+0 .
$$

Покажем, что при любом $\varepsilon>0$ и $y \in(0,1)$ функция $L_{g}^{\omega}(x, y)$ измерима по $x$ как функция со значениями в $G_{\varepsilon}$. Пусть

$$
\eta(k) \in C^{\infty}, \quad \eta(k)= \begin{cases}1, & 0<k<1 \\ 0, & k>2\end{cases}
$$

Тогда функции $\eta(k / n) L_{g}^{\omega}(x, y)=\eta(k / n) L_{f}^{\omega}(x / k, y / k), n=1,2, \ldots$, непрерывны по $x$ как функции со значениями в $G_{\varepsilon}$. Пользуясь оценкой $(3.11)$, имеем

$$
\begin{aligned}
\left\|L_{g}^{\omega}(x, y)-\eta\left(\frac{k}{n}\right) L_{g}^{\omega}(x, y)\right\|_{G_{\varepsilon}} & =\sup _{k>1} \frac{1}{k^{\varepsilon} \rho(k)}\left\|L_{f}^{\omega}\left(\frac{x}{k}, \frac{y}{k}\right)-L_{f}^{\omega}\left(\frac{x}{k}, \frac{y}{k}\right) \eta\left(\frac{k}{n}\right)\right\|_{\mathbb{B}} \\
& \leqslant \sup _{k>n} \frac{1}{k^{\varepsilon} \rho(k)}\left|\eta\left(\frac{k}{n}\right)-1\right|\left\|L_{f}^{\omega}\left(\frac{x}{k}, \frac{y}{k}\right)\right\|_{\mathbb{B}} \\
& \leqslant \frac{1}{n^{\varepsilon}} \sup _{k>1} \frac{1}{\rho(k)}\left\|L_{f}^{\omega}\left(\frac{x}{k}, \frac{y}{k}\right)\right\|_{\mathbb{B}} \underset{n \rightarrow \infty}{\longrightarrow} 0 .
\end{aligned}
$$

Пользуясь теперь теоремой 2.1 , заключаем, что сушествует $\ell$ такое, что

$$
g \in L\left(\tilde{\mathscr{S}}_{\left[\ell ; P_{0}, \ldots, P_{\ell}\right]} \rightarrow G\right) .
$$

Откуда и вытекает (3.8). Лемма доказана.

ЗАмечаниЕ 1. Пусть в условиях леммы существует еще

$$
\lim _{k \rightarrow+\infty} \frac{1}{\rho(k)} L_{f}^{\omega}\left(\frac{x}{k}, \frac{y}{k}\right)=C(x, y), \quad|x|^{2}+y^{2}=1, \quad y>0 .
$$

Тогда $f(x)$ обладает квазиасимптотикой в нуле относительно $\rho(k)$, т.е. существует

$$
\lim _{k \rightarrow+\infty}\left(f\left(\frac{\xi}{k}\right), \varphi(\xi)\right)=c_{\varphi} \quad \forall \varphi \in \widetilde{\mathscr{T}}_{\left[\ell ; P_{0}, P_{1}, \ldots, P_{\ell}\right]} .
$$

Доказательство аналогично, следует только в качестве пространства $G$ использовать пространство

$$
G=\left\{\gamma(k) \in C([1, \infty) \rightarrow \mathbb{B}): \exists \lim _{k \rightarrow+\infty} \frac{1}{\rho(k)}\|\gamma(k)\|_{\mathbb{B}}\right\}
$$

с той же нормой и учесть, что в этом случае измеримость $L_{g}^{\omega}(x, y)$ по $x$ как функций со значениями в $G$ будет следовать из леммы П.6 работы [1].

ЗАмечАниЕ 2. Если в условиях леммы и замечании 1 заменить $L_{f}^{\omega}(x / k, y / k)$ на $L_{f}^{\omega}(k x, k y)($ см. $(3.7)$ и $(3.14))$, то утверждения леммы и замечание к ней останутся справедливыми, если только вместо $f(\xi / k)$ всюду подставить $f(k \xi)$. Другими словами, в этом случае $f$ будет квазиасимптотически ограничена (обладать квазиасимптотикой) на бесконечности относительно $\rho(k)$ на множестве $\widetilde{\mathscr{S}}_{\left[\ell ; P_{0}, P_{1}, \ldots, P_{\ell}\right]}$ при некотором $\ell$. 
Лемма 3.2. Пусть $\mathbb{B}$ - банахово пространство, $g(t) \in \mathbb{L}(\mathscr{S} \rightarrow \mathbb{B})$ u $\rho(k)-$ автомодельная функиия порядка $\alpha$. Пусть также существует целое неотрицательное число $\ell$ такое, что $g(t)$ квазиасимптотически ограничена (обладает квазиасимптотикой) в нуле относительно $\rho(k)$ на основных функииях $\varphi(t) \in \mathscr{S}$ таких, что $T_{\varphi}^{\ell-1}(t) \equiv 0$, m.e.

$$
\begin{array}{ll}
\sup _{k \geqslant 1} \frac{1}{\rho(k)}\left\|\left(g\left(\frac{t}{k}\right), \varphi(t)\right)\right\|_{\mathbb{B}}<\infty & \forall \varphi \in \mathscr{S}, \quad T_{\varphi}^{\ell-1}(t) \equiv 0 \\
\left(\lim _{k \rightarrow+\infty} \frac{1}{\rho(k)}\left(g\left(\frac{t}{k}\right), \varphi(t)\right)=C_{\varphi}\right. & \left.\forall \varphi \in \mathscr{S}, \quad T_{\varphi}^{\ell-1}(t) \equiv 0\right) .
\end{array}
$$

Тогда если $\alpha \neq n, n+1, \ldots$, то существуют постоянние

$$
C_{j} \in \mathbb{B}, \quad j=\left(j_{1}, \ldots, j_{n}\right), \quad|j|<\ell
$$

такие, что обобщенная функиия

$$
G(t)=g(t)-\sum_{|j|<\ell} C_{j} \delta^{(j)}(t)
$$

квазиасимптотически ограничена (обладает квазиасимптотикой) в нуле относительно $\rho(k)$ на $\mathscr{S}$, m.е.

$$
\begin{array}{ll}
\sup _{k \geqslant 1} \frac{1}{\rho(k)}\left\|\left(G\left(\frac{t}{k}\right), \varphi(t)\right)\right\|_{\mathbb{B}}<\infty & \forall \varphi \in \mathscr{S} \\
\left(\lim _{k \rightarrow+\infty} \frac{1}{\rho(k)}\left(G\left(\frac{t}{k}\right), \varphi(t)\right)=C_{\varphi}\right. & \forall \varphi \in \mathscr{S}) .
\end{array}
$$

Eсли же $\alpha=n+p$, где $p=0,1, \ldots$, mo

$$
\begin{array}{ll}
\sup _{k \geqslant 1} \frac{1}{\rho(k)}\left\|\left(G\left(\frac{t}{k}\right), \varphi(t)\right)\right\|_{\mathbb{B}}<\infty & \forall \varphi \in \mathscr{S}, \quad T_{\varphi}^{p}(t) \equiv 0 \\
\left(\lim _{k \rightarrow+\infty} \frac{1}{\rho(k)}\left(G\left(\frac{t}{k}\right), \varphi(t)\right)=C_{\varphi} \quad \forall \varphi \in \mathscr{S}, \quad T_{\varphi}^{p}(t) \equiv 0\right),
\end{array}
$$

$2 \partial e$

$$
G(t)=g(t)-\sum_{|j|<\ell} C_{j} \delta^{(j)}(t)
$$

с некоторьми $C_{j} \in \mathbb{B}$.

ДоКАЗАТЕЛЬСтво. Мы рассмотрим лишш случай квазиасимптотической ограниченности. Доказательство в случае существования квазиасимптотики совершенно аналогично. Без ограничения общности можно считать, что $n+\ell-1>\alpha$. По индукции мы построим семейство обобщенных функций

$$
\left\{g_{\ell}(t), g_{\ell-1}(t), \ldots, g_{s}(t), \ldots, g_{s_{0}}(t)\right\}, \quad s_{0} \geqslant 0
$$

таких, что $g_{s}(t), s=\ell, \ell-1, \ldots, s_{0}$, отличается от $g(t)$ на обобщенную функцию вида $\sum_{|j|<\ell} C_{j} \delta^{(j)}(t)$ с некоторыми $C_{j} \in \mathbb{B}$ и каждая $g_{s}(t)$ квазиасимптотически 
ограничена в нуле относительно $\rho(k)$ на всех $\varphi(t) \in \mathscr{S}$ таких, что $T_{\varphi}^{s-1}(t) \equiv 0$. То есть

$$
\sup _{k \geqslant 1} \frac{1}{\rho(k)}\left\|\left(g_{s}\left(\frac{t}{k}\right), \varphi(t)\right)\right\|_{\mathbb{B}}<\infty \quad \forall \varphi \in \mathscr{S}, T_{\varphi}^{s-1}(t) \equiv 0 .
$$

Число $s_{0}$ определится в процессе построения.

Положим $g_{\ell}(t)=g(t)$ и предположим, что $g_{\ell}, g_{\ell-1}, \ldots, g_{s}$ уже построены. Построим функцию $g_{s-1}(t)$. При этом могут встретиться три случая: $n+s-1>\alpha$, $n+s-1<\alpha$ и $n+s-1=\alpha$.

Случай $1: n+s-1>\alpha$. Пусть $j$ - мультиндекс такой, что $|j|=s-1$. Непосредственной проверкой (дифференциируя по $k$ ) можно убедиться, что

$$
\begin{aligned}
\frac{1}{k^{n+s-1}}\left(g_{s}\left(\frac{t}{k}\right), t^{j} \varphi(t)\right)= & -\int_{k}^{\infty}\left(g_{s}\left(\frac{t}{\varkappa}\right), t^{j} \sum_{q=1}^{n} t_{q} \frac{\partial}{\partial t_{q}} \varphi(t)\right) \frac{d \varkappa}{\varkappa^{n+s}} \\
& +\left(\gamma_{j}(t), \varphi(t)\right) \quad \forall \varphi \in \mathscr{S}
\end{aligned}
$$

где $\gamma_{j}(t) \in \mathbb{L}(\mathscr{S} \rightarrow \mathbb{B})$. Согласно индуктивному предположению (3.22) интеграл справа в (3.23) сходится. Подставляя в (3.23) вместо $\varphi$ функции $t_{q} \psi(t)$, где $q=$ $1, \ldots, n, \psi \in \mathscr{S}$, учитывая, что $T_{t^{j} t_{q} \psi}^{s-1}(t) \equiv 0$, и соотношение $(3.22)$, при $k \rightarrow \infty$ имеем $\left(\gamma_{j}(t), t_{q} \psi(t)\right)=0$. Следовательно,

$$
\gamma_{j}(t)=C_{j} \delta(t), \quad C_{j} \in \mathbb{B} .
$$

Положим $g_{s-1}(t)=g_{s}(t)-\sum_{|i|=s-1}(1 / i !) C_{i} \delta^{(i)}(t)$. Докажем, что $g_{s-1}(t)$ квазиасимптотически ограничена в нуле относительно $\rho(k)$ на всех $\varphi \in \mathscr{S}$ таких, что $T_{\varphi}^{s-2}(t) \equiv 0$. Для этого заметим, что

$$
\begin{aligned}
\left(g_{s-1}\left(\frac{t}{k}\right), t^{j} \varphi(t)\right) & =\left(g_{s}\left(\frac{t}{k}\right), t^{j} \varphi(t)\right)-\left(\frac{1}{j !} C_{j} \delta^{(j)}\left(\frac{t}{k}\right), t^{j} \varphi(t)\right) \\
& =\left(g_{s}\left(\frac{t}{k}\right), t^{j} \varphi(t)\right)-k^{s-1}\left(C_{j} \delta\left(\frac{t}{k}\right), \varphi(t)\right) \\
& =\left(g_{s}\left(\frac{t}{k}\right), t^{j} \varphi(t)\right)-k^{n+s-1}\left(\gamma_{j}(t), \varphi(t)\right) .
\end{aligned}
$$

Мы воспользовались тем, что при $|j|=|i|$

$$
t^{j} \delta^{(i)}(t)= \begin{cases}0, & i \neq j \\ j ! \delta(t), & i=j .\end{cases}
$$

Используя (3.24) из соотношения (3.23), получаем

$$
\begin{gathered}
\frac{1}{\rho(k)}\left(g_{s-1}\left(\frac{t}{k}\right), t^{j} \varphi(t)\right)=-\frac{k^{n+s-1}}{\rho(k)} \int_{k}^{\infty}\left(g_{s}\left(\frac{t}{\varkappa}\right), t^{j} \sum_{q=1}^{n} t_{q} \frac{\partial}{\partial t_{q}} \varphi(t)\right) \frac{d \varkappa}{\varkappa^{n+\ell}} \\
\forall \varphi \in \mathscr{S} .
\end{gathered}
$$

В силу (3.22)

$$
\frac{1}{\varkappa^{n+s}}\left\|\left(g_{s}\left(\frac{t}{\varkappa}\right), t^{j} \sum_{q=1}^{n} t_{q} \frac{\partial}{\partial t_{q}} \varphi(t)\right)\right\|_{\mathbb{B}} \leqslant \operatorname{const} \frac{\rho(\varkappa)}{\varkappa^{n+s}} \quad \forall \varphi \in \mathscr{S} .
$$


Поэтому из (3.25) следует неравенство

$$
\frac{1}{\rho(k)}\left\|\left(g_{s-1}\left(\frac{t}{k}\right), t^{j} \varphi(t)\right)\right\|_{\mathbb{B}} \leqslant \operatorname{const} \frac{k^{n+s-1}}{\rho(k)} \int_{k}^{+\infty} \frac{\rho(\varkappa)}{\varkappa^{n+s}} d \varkappa
$$

Так как порядок автомодельности функции $\rho(\varkappa) / \varkappa^{n+s}$ равен $\alpha-n-s<-1$, то в силу (1.11) из (3.26) заключаем, что $g_{s-1}(t)$ квазиасимптотически ограничена в нуле относительно $\rho(k)$ на всех основных функциях вида $t^{j} \varphi(t),|j|=s-1, \varphi \in \mathscr{S}$. Пусть $\varphi(t) \in \mathscr{S}$ такова, что $T_{\varphi}^{s-2}(t) \equiv 0$. Положим

$$
\eta(t) \in \mathscr{S}, \quad \eta(t)= \begin{cases}1, & |t|<1 \\ 0, & |t|>2\end{cases}
$$

Имеем

$$
\begin{aligned}
\frac{1}{\rho(k)}\left(g_{s-1}\left(\frac{t}{k}\right), \varphi(t)\right)= & \frac{1}{\rho(k)}\left(g_{s-1}\left(\frac{t}{k}\right), \eta(t) \varphi(t)\right) \\
& +\frac{1}{\rho(k)}\left(g_{s-1}\left(\frac{t}{k}\right),(1-\eta(t)) \varphi(t)\right)
\end{aligned}
$$

Второе слагаемое справа можно переписать в виде $(1 / \rho(k))\left(g_{s}(t / k),(1-\eta(t)) \varphi(t)\right)$, если заметить, что

$$
\left(g_{s-1}(t), \psi(t)\right)=\left(g_{s}(t), \psi(t)\right) \quad \forall \psi \in \mathscr{S}, \quad T_{\psi}^{s-1}(t) \equiv 0
$$

Поэтому в силу квазиасимптотической ограниченности $g_{s}$ на таких основных функциях (см. (3.22)) это слагаемое ограничено при $k \rightarrow+\infty$. Первое слагаемое справа в (3.27) в силу (3.28) можно переписать следующим образом:

$$
\begin{aligned}
& \frac{1}{\rho(k)}\left(g_{s-1}\left(\frac{t}{k}\right), \eta(t)\left(\varphi(t)-T_{\varphi}^{s-1}(t)\right)\right)+\frac{1}{\rho(k)}\left(g_{s-1}\left(\frac{t}{k}\right), \eta(t) T_{\varphi}^{s-1}(t)\right) \\
& =\frac{1}{\rho(k)}\left(g_{s}\left(\frac{t}{k}\right), \eta(t)\left(\varphi(t)-T_{\varphi}^{s-1}(t)\right)\right)+\frac{1}{\rho(k)}\left(g_{s-1}\left(\frac{t}{k}\right), \eta(t) T_{\varphi}^{s-1}(t)\right)
\end{aligned}
$$

Первое слагаемое справа в (3.29) ограничено по индуктивному предположению (3.22), а второе слагаемое ограничено по уже доказанному, так как $T_{\varphi}^{s-1}(t)$ - однородный многочлен степени $s-1$. Таким образом, построенная нами $g_{s-1}$ обладает требуемыми свойствами.

Случай $2: n+s-1<\alpha$. Положим $g_{s-1}(t)=g_{s}(t)$. Докажем, что $g_{s-1}(t)$ квазиасимптотически ограничена в нуле относительно $\rho(k)$ на всех $\varphi \in \mathscr{S}$ таких, что $T_{\varphi}^{s-2}(t) \equiv 0$. Пусть $j-$ мультиндекс такой, что $|j|=s-1$. Теперь вместо $(3.23)$ воспользуемся легко проверяемой формулой

$\frac{1}{k^{n+s-1}}\left(g_{s}\left(\frac{t}{k}\right), t^{j} \varphi(t)\right)=\left(g_{s}(t), t^{j} \varphi(t)\right)+\int_{1}^{k}\left(g_{s}\left(\frac{t}{\varkappa}\right), t^{j} \sum_{q=1}^{n} t_{q} \frac{\partial}{\partial t_{q}} \varphi(t)\right) \frac{d \varkappa}{\varkappa^{n+s}}$. 
Из нее следует, что

$$
\begin{aligned}
& \frac{1}{\rho(k)}\left(g_{s-1}\left(\frac{t}{k}\right), t^{j} \varphi(t)\right)=\frac{k^{n+s-1}}{\rho(k)}\left(g_{s}(t), t^{j} \varphi(t)\right) \\
& \quad+\frac{k^{n+s-1}}{\rho(k)} \int_{1}^{k}\left(g_{s}\left(\frac{t}{\varkappa}\right), t^{j} \sum_{q=1}^{n} t_{q} \frac{\partial}{\partial t_{q}} \varphi(t)\right) \frac{d \varkappa}{\varkappa^{n+s}} \quad \forall \varphi \in \mathscr{S} .
\end{aligned}
$$

Первое слагаемое справа при $k \rightarrow+\infty$ стремится к нулю, а второе слагаемое ограничено в силу следующей оценки:

$$
\begin{aligned}
& \frac{k^{n+s-1}}{\rho(k)} \int_{1}^{k}\left\|\left(g_{s}\left(\frac{t}{\varkappa}\right), t^{j} \sum_{q=1}^{n} t_{q} \frac{\partial}{\partial t_{q}} \varphi(t)\right)\right\|_{\mathbb{B}} \frac{d \varkappa}{\varkappa^{n+s}} \\
& \leqslant \operatorname{const} \frac{k^{n+s-1}}{\rho(k)} \int_{1}^{k} \frac{\rho(\varkappa)}{\varkappa^{n+s}} d \varkappa \leqslant \text { const. }
\end{aligned}
$$

Здесь мы воспользовались индуктивньм предположением (3.22), имея в виду, что тейлоровское разложение в нуле порядка $s-1$ функции $t^{j} \sum_{q=1}^{n} t_{q} \partial \varphi(t) / \partial t$ тождественно равно нулю, а также свойством автомодельных функций (1.11), учитывая, что порядок автомодельной функции $\rho(\varkappa) / \varkappa^{n+s}$ равен $\alpha-n-s>-1$. Таким образом, мы показали, что $g_{s-1}(t)$ квазиасимптотически ограничена в нуле относительно $\rho(k)$ на функциях вида $t^{j} \varphi(t),|j|=s-1, \varphi \in \mathscr{S}$. Далее, точно так же, как и в предыдушем случае, показывается, что она квазиасимптотически ограничена и на всех $\varphi \in \mathscr{S}$ таких, что $T_{\varphi}^{s-2}(t) \equiv 0$.

Случай 3: $n+s-1=\alpha$. В этом случае мы полагаем $s=s_{0}$ и завершаем построение.

Продолжая доказательство леммы, положим $G(t)=g_{s_{0}}(t)$. Ясно, что если $\alpha \neq n, n+1, \ldots$, то $s_{0}=0$ и $G(t)$ удовлетворяет соотношениям (3.18) и (3.19). Если же $\alpha=n+p$, где $p=0,1, \ldots$, то $s_{0}=p+1$, а стало быть, $G(t)$ удовлетворяет соотношению (3.20). Лемма доказана.

ЗАмечАнИЕ. Отметим, что в формуле (3.18) в сумме справа можно оставить лишь те члены, для которых $|j|>\alpha-n$, поскольку

$$
\frac{1}{\rho(k)} \delta^{(j)}\left(\frac{t}{k}\right)=\frac{k^{n+|j|}}{\rho(k)} \underset{k \rightarrow+\infty}{\longrightarrow} 0, \quad|j|<\alpha-n
$$

Точно так же в (3.21) можно оставить лишь те слагаемые, для которых $|j|>p$, так как если $|j|<p$, то

$$
\frac{1}{\rho(k)} \delta^{(j)}\left(\frac{t}{k}\right)=\frac{k^{n+|j|}}{\rho(k)} \underset{k \rightarrow+\infty}{\longrightarrow} 0 .
$$

Если же $|j|=p$, то $\left(\delta^{(j)}(t / k), \varphi(t)\right)=0$ для $\varphi \in \mathscr{S}$ таких, что $T_{\varphi}^{p}(t) \equiv 0$.

Лемма 3.3. Пусть $\mathbb{B}-$ банахово пространство, $g(t) \in \mathbb{L}(\mathscr{S} \rightarrow \mathbb{B})$ u $\rho(k)$ - автомодельная функиия порядка $\alpha$. Пусть также существует иелое неотрицательное число $\ell$ такое, что $g(t)$ квазиасимптотически ограничена 
(обладает квазиасимптотикой) на бесконечности относительно $\rho(k)$ на основных функциях $\varphi(t) \in \mathscr{S}$ таких, что $T_{\varphi}^{\ell-1}(t) \equiv 0$, m.е.

$$
\begin{array}{ll}
\sup _{k \geqslant 1} \frac{1}{\rho(k)}\|(g(k t), \varphi(t))\|_{\mathbb{B}}<\infty & \forall \varphi \in \mathscr{S}, \quad T_{\varphi}^{\ell-1}(t) \equiv 0 \\
\left(\lim _{k \rightarrow+\infty}(g(k t), \varphi(t))=C_{\varphi} \quad \forall \varphi \in \mathscr{S}, \quad T_{\varphi}^{\ell-1}(t) \equiv 0\right) .
\end{array}
$$

Тогда если $\alpha \neq-n,-n-1, \ldots$, то существуют постояннье $C_{j} \in \mathbb{B}, j=$ $\left(j_{1}, \ldots, j_{n}\right),|j|<\ell$, такие, что обобщенная функиия

$$
G(t)=g(t)-\sum_{|j|<\ell} C_{j} \delta^{(j)}(t)
$$

квазиасимптотически ограничена (обладает квазиасимптотикой) на бесконечности относительно $\rho(k)$ на $\mathscr{S}$, т.е.

$$
\begin{array}{ll}
\sup _{k \geqslant 1} \frac{1}{\rho(k)}\|(G(k t), \varphi(t))\|_{\mathbb{B}}<\infty & \forall \varphi \in \mathscr{S} \\
\left(\lim _{k \rightarrow+\infty}(G(k t), \varphi(t))=C_{\varphi}\right. & \forall \varphi \in \mathscr{S}) .
\end{array}
$$

Если же $\alpha=-n-p$, где $p=0,1, \ldots$, то $g(t)$ квазиасимптотически ограничена (обладает квазиасимптотикой) на бесконечности относительно $\rho(k)$ на всех $\varphi \in \mathscr{S}$ maких, что $T_{\varphi}^{p}(t) \equiv 0$, m.e.

$$
\begin{array}{lll}
\sup _{k \geqslant 1} \frac{1}{\rho(k)}\|(g(k t), \varphi(t))\|_{\mathbb{B}}<\infty & \forall \varphi \in \mathscr{S}, \quad T_{\varphi}^{p}(t) \equiv 0 \\
\left(\lim _{k \rightarrow+\infty}(g(k t), \varphi(t))=C_{\varphi}\right. & \left.\forall \varphi \in \mathscr{S}, \quad T_{\varphi}^{p}(t) \equiv 0\right) .
\end{array}
$$

ДокАЗАТЕльство. Так же, как и при доказательстве предыдущей леммы, мы ограничимся случаем квазиасимптотической ограниченности. Доказательство в случае сушествования квазиасимптотики совершенно аналогично. Без ограничения обшности можно считать $\ell$ настолько большим, что $n+\ell-1>-\alpha$. По индукции мы построим семейство обобшенных функций

$$
\left\{g_{\ell}(t), g_{\ell-1}(t), \ldots, g_{s}(t), \ldots, g_{s_{0}}(t)\right\}, \quad s_{0} \geqslant 0
$$

таких, что $g_{s}(t), s=\ell, \ell-1, \ldots, s_{0}$, отличается от $g(t)$ на обобшенную функцию вида $\sum_{|j|<\ell} C_{j} \delta^{(j)}(t)$ с некоторыми $C_{j} \in \mathbb{B}$ и каждая $g_{s}(t)$ квазиасимптотически ограничена на бесконечности относительно $\rho(k)$ на всех $\varphi(t) \in \mathscr{S}$ таких, что $T_{\varphi}^{s-1}(t) \equiv 0$. То есть

$$
\sup _{k \geqslant 1} \frac{1}{\rho(k)}\left\|\left(g_{s}(k t), \varphi(t)\right)\right\|_{\mathbb{B}}<\infty \quad \forall \varphi \in \mathscr{S}, \quad T_{\varphi}^{s-1}(t) \equiv 0 .
$$

Число $s_{0}$ определится в процессе построения.

Положим $g_{\ell}(t)=g(t)$ и предположим, что $g_{\ell}, g_{\ell-1}, \ldots, g_{s}$ уже построены. Построим функцию $g_{s-1}(t)$. При этом могут встретиться три случая: $n+s-1+\alpha>0$, $n+s-1+\alpha<0$ и $n+s-1+\alpha=0$. 
Случай 1: $n+s-1+\alpha>0$. Положим $g_{s-1}(t)=g_{s}(t)$. Докажем, что $g_{s-1}(t)$ квазиасимптотически ограничена на бесконечности относительно $\rho(k)$ на всех $\varphi \in \mathscr{S}$ таких, что $T_{\varphi}^{s-2}(t) \equiv 0$. Пусть $j$ - мультиндекс такой, что $|j|=s-1$. Воспользуемся легко проверяемой (дифференциированием по $k$ ) формулой

$k^{n+s-1}\left(g_{s}(k t), t^{j} \varphi(t)\right)=\left(g_{s}(t), t^{j} \varphi(t)\right)-\int_{1}^{k}\left(g_{s}(\varkappa t), t^{j} \sum_{q=1}^{n} t_{q} \frac{\partial}{\partial t_{q}} \varphi(t)\right) \varkappa^{n+s-2} d \varkappa$.

Из нее следует, что

$$
\begin{aligned}
& \frac{1}{\rho(k)}\left(g_{s-1}(k t), t^{j} \varphi(t)\right)=\frac{1}{k^{n+s-1} \rho(k)}\left(g_{s}(t), t^{j} \varphi(t)\right) \\
& \quad-\frac{1}{k^{n+s-1} \rho(k)} \int_{1}^{k}\left(g_{s}(\varkappa t), t^{j} \sum_{q=1}^{n} t_{q} \frac{\partial}{\partial t_{q}} \varphi(t)\right) \varkappa^{n+s-2} d \varkappa \quad \forall \varphi \in \mathscr{S} .
\end{aligned}
$$

Первое слагаемое справа при $k \rightarrow+\infty$ стремится к нулю, а второе слагаемое ограничено в силу следуюшей оценки:

$$
\begin{gathered}
\frac{1}{k^{n+s-1} \rho(k)} \int_{1}^{k}\left\|\left(g_{s}(\varkappa t), t^{j} \sum_{q=1}^{n} t_{q} \frac{\partial}{\partial t_{q}} \varphi(t)\right)\right\|_{\mathbb{B}} \varkappa^{n+s-2} d \varkappa \\
\leqslant \operatorname{const} \frac{1}{k^{n+s-1} \rho(k)} \int_{1}^{k} \rho(\varkappa) \varkappa^{n+s-2} d \varkappa \leqslant \text { const. }
\end{gathered}
$$

Здесь мы воспользовались индуктивным предположением (3.35), имея в виду, что тейлоровское разложение в нуле порядка $s-1$ функции $t^{j} \sum_{q=1}^{n} t_{q} \partial \varphi(t) / \partial t$ тождественно равно нулю, а также свойством автомодельных функций (1.11), учитывая, что порядок автомодельной функции $\rho(\varkappa) \varkappa^{n+s-2}$ равен $\alpha-n-s-2>-1$. Таким образом, мы показали, что $g_{s-1}(t)$ квазиасимптотически ограничена на бесконечности относительно $\rho(k)$ на функциях вида $t^{j} \varphi(t),|j|=s-1, \varphi \in \mathscr{S}$. Далее, рассуждая, как и в предыдушей лемме (пользуясь формулами (3.27)-(3.29), в которых следует только заменить $t / k$ на $k t)$, показываем, что $g_{s-1}(t)$ квазиасимптотически ограничена на бесконечности относительно $\rho(k)$ на всех функциях $\varphi \in \mathscr{S}$ таких, что $T_{\varphi}^{s-2}(t) \equiv 0$.

Случай $2: n+s-1+\alpha<0$. Пусть $j$ - мультиндекс такой, что $|j|=s-1$. Непосредственной проверкой (дифференциируя по $k$ ) можно убедиться, что

$$
\begin{aligned}
k^{n+s-1}\left(g_{s}(k t), t^{j} \varphi(t)\right) & =\int_{k}^{\infty}\left(g_{s}(t \varkappa), t^{j} \sum_{q=1}^{n} t_{q} \frac{\partial}{\partial t_{q}} \varphi(t)\right) \varkappa^{n+s-2} d \varkappa \\
& +\left(\gamma_{j}(t), \varphi(t)\right) \quad \forall \varphi \in \mathscr{S},
\end{aligned}
$$

где $\gamma_{j}(t) \in \mathbb{L}(\mathscr{S} \rightarrow \mathbb{B})$. Согласно индуктивному предположению (3.35)

$$
\left\|\left(g_{s}(t \varkappa), t^{j} \sum_{q=1}^{n} t_{q} \frac{\partial}{\partial t_{q}} \varphi(t)\right)\right\|_{\mathbb{B}} \leqslant \operatorname{const} \rho(\varkappa),
$$

а так как функция $\rho(\varkappa) \varkappa^{n+s-2}$ имеет порядок $n+s-2+\alpha<-1$, то интеграл справа в (3.37) сходится. Подставляя в (3.37) вместо $\varphi$ функции $t_{q} \psi(t)$, где $q=1, \ldots, n$, 
$\psi \in \mathscr{S}$, учитывая, что $T_{t^{j} t_{q} \psi}^{s-1}(t) \equiv 0$, и соотношение $(3.35)$, при $k \rightarrow \infty$ имеем $\left(\gamma_{j}(t), t_{q} \psi(t)\right)=0$. Следовательно,

$$
\gamma_{j}(t)=C_{j} \delta(t), \quad C_{j} \in \mathbb{B} \text {. }
$$

Положим $g_{s-1}(t)=g_{s}(t)-\sum_{|i|=s-1}(1 / i !) C_{i} \delta^{(i)}(t)$. Из соотношения $(3.37)$, пользуясь формулой (3.24), в которой $k$ заменено на $1 / k$, получаем

$$
\begin{gathered}
\frac{1}{\rho(k)}\left(g_{s-1}(k t), t^{j} \varphi(t)\right)=\frac{1}{k^{n+s-1} \rho(k)} \int_{k}^{+\infty}\left(g_{s}(\varkappa t), t^{j} \sum_{q=1}^{n} t_{q} \frac{\partial}{\partial t_{q}} \varphi(t)\right) \varkappa^{n+s-2} d \varkappa \\
\forall \varphi \in \mathscr{S} .
\end{gathered}
$$

Отсюда, замечая, что порядок автомодельной функции $\rho(\varkappa) \varkappa^{n+s-2}$ равен $\alpha+n+$ $s-2<-1$, как и в предыдущей лемме, заключаем, что $g_{s-1}(t)$ квазиасимптотически ограничена на бесконечности относительно $\rho(k)$ на всех $\varphi \in \mathscr{S}$ таких, что $T_{\varphi}^{s-2}(t) \equiv 0$.

Случай 3: $n+s-1+\alpha=0$. В этом случае мы полагаем $s=s_{0}$ и завершаем построение.

Продолжая доказательство леммы, положим $G(t)=g_{s_{0}}(t)$. Ясно, что если $\alpha \neq-n,-n-1, \ldots$, то $s_{0}=0$ и $G(t)$ удовлетворяет соотношениям (3.32) и (3.33). Если же $\alpha=-n-p$, где $p=0,1, \ldots$, то $s_{0}=p+1$, а стало быть, $G(t)=g(t)$ и $g(t)$ удовлетворяет соотношению (3.34). Лемма доказана.

ЗАмечАниЕ. Отметим, что в формуле (3.32) в сумме справа можно оставить лишь те члены, для которых $|j|<-\alpha-n$, так как

$$
\frac{1}{\rho(k)} \delta^{(j)}(k t)=\frac{1}{k^{n+|j|} \rho(k)} \underset{k \rightarrow+\infty}{\longrightarrow} 0, \quad|j|>-\alpha-n .
$$

ТЕОРема 3.2. Пусть $\mathbb{B}$ - банахово пространство, $\rho(k)$ - автомодельная функиия порядка $\alpha, f \in \mathbb{L}(\mathscr{S} \rightarrow \mathbb{B}) u \omega(x) \in \mathscr{S}$, причем $\widetilde{\omega}(t)$ не вырождена в нуле, а ее тейлоровское разложение в начале координат имеет вид:

$$
T_{\widetilde{\omega}}^{N}(t)=\sum_{q=0}^{N} P_{q}(t), \quad N=0,1, \ldots
$$

где $P_{q}(t)$ - однородный многочлен степени $q$ (либо тожддественный нуль). Пусть еще существуют постоянные $A$ и $b$ такие, что

$$
\sup _{k>1} \frac{1}{\rho(k)}\left\|L_{f}^{\omega}(k x, k y)\right\|_{\mathbb{B}} \leqslant \frac{A}{y^{b}}, \quad|x|^{2}+y^{2}=1, \quad y>0 .
$$

Тогда существует единственный многочлен вида

$$
Q(x)=\sum_{\alpha<\tau<\ell} Q_{\tau}(x)
$$

где $Q_{\tau}(x)$ - однородный многочлен степени $\tau$ (либо тожсдественный нуль), $\ell$ - некоторое целое число, такой, что обобщенная функиия $G(x)=f(x)-Q(x)$ квазиасимптотически ограничена на бесконечности относительно $\rho(k)$ на 
всех $\varphi \in \mathscr{S}$, если $\alpha \neq 0,1,2, \ldots$, ина всех $\varphi \in \widetilde{\mathscr{S}}_{\left[\alpha ; P_{0}, P_{1}, \ldots, P_{\alpha}\right]}$, если $\alpha=0,1, \ldots$ To ecmb

$$
\sup _{k>1} \frac{1}{\rho(k)}\|(G(k x), \varphi(x))\|_{\mathbb{B}}<\infty \quad \begin{cases}\forall \varphi \in \mathscr{S}, & \text { ecлu } \alpha \neq 0,1, \ldots \\ \forall \varphi \in \widetilde{\mathscr{S}}_{\left[\alpha ; P_{0}, \ldots, P_{\alpha}\right]}, & \text { ecли } \alpha=0,1, \ldots\end{cases}
$$

При этом

$$
P_{q}\left(\frac{\partial}{\partial x}\right) Q_{\tau}(x)=0, \quad \alpha<\tau<\ell, \quad q=0,1, \ldots
$$

ДокАЗАТЕЛЬство. Из замечания 2 к лемме 3.1 , леммы 3.2 и замечания к ней вытекает, что сушествует многочлен вида (3.40) такой, что для $G(x)=f(x)-Q(x)$ справедливо неравенство

$$
\sup _{k>1} \frac{1}{\rho(k)}\|(G(k \xi), \varphi(\xi))\|_{\mathbb{B}}<\infty \quad \begin{cases}\forall \varphi \in \mathscr{S}, & \alpha \neq 0,1, \ldots \\ \forall \varphi \in \mathscr{S}, T_{\tilde{\varphi}}^{\alpha}(t) \equiv 0, & \alpha=0,1, \ldots\end{cases}
$$

Чтобы в этом убедиться, достаточно в лемме 3.2 положить $g(t)=\widetilde{f}(t)$ и учесть, что

$$
F\left[\frac{1}{\rho(k)} f(k x)\right]=\frac{1}{k^{n} \rho(k)} \tilde{f}\left(\frac{t}{k}\right) .
$$

Докажем, что на самом деле для этого же многочлена справедливо неравенство (3.41). Для этого, конечно, достаточно рассмотреть случай $\alpha=0,1, \ldots$.

Пусть $\widetilde{\varphi}(t) \in \mathscr{S}_{\left[\alpha ; P_{0}, \ldots, P_{\alpha}\right]}$, тогда

$$
\begin{aligned}
\frac{1}{\rho(k)}(G(k x), \varphi(x))= & \frac{1}{k^{n} \rho(k)}\left(g\left(\frac{t}{k}\right)-\sum_{\alpha<|j|} C_{j} \delta^{(j)}\left(\frac{t}{k}\right), \widetilde{\varphi}(t)(1-h(t))\right) \\
& +\frac{1}{k^{n} \rho(k)}\left(g\left(\frac{t}{k}\right)-\sum_{\alpha<|j|} C_{j} \delta^{(j)}\left(\frac{t}{k}\right),\left(\widetilde{\varphi}(t)-T_{\widetilde{\varphi}}^{\alpha}(t)\right) h(t)\right) \\
& +\frac{1}{k^{n} \rho(k)}\left(g\left(\frac{t}{k}\right)-\sum_{\alpha<|j|} C_{j} \delta^{(j)}\left(\frac{t}{k}\right), T_{\widetilde{\varphi}}^{\alpha}(t) h(t)\right)
\end{aligned}
$$

где $h(t) \in \mathscr{S}, h(t)=1$ при $|t|<1$. Первые два слагаемых справа в (3.43) ограничены, так как тейлоровское разложение в нуле порядка $\alpha$ соответствующих основных функций в этих слагаемых тождественно равно нулю. А третье слагаемое равно

$$
\left(g\left(\frac{t}{k}\right), T_{\widetilde{\varphi}}^{\alpha}(t) h(t)\right)=\frac{1}{\rho(k)}\left(f(k x), F^{-1}\left[h(t) T_{\widetilde{\varphi}}^{\alpha}(t)\right](x)\right) .
$$

Мы учли, что $\left(\sum_{\alpha<|j|} C_{j} \delta^{(j)}(t), T_{\widetilde{\varphi}}^{\alpha}(t) h(t)\right)=0$. Так как $T_{\widetilde{\varphi}}^{\alpha}(t) h(t) \in \mathscr{I}_{\left[\ell ; P_{0}, \ldots, P_{\ell}\right]}$ для любого $\ell \geqslant \alpha$, то по замечанию 2 к лемме 3.1 оно также ограничено. Что и требовалось.

Докажем единственность многочлена (3.40). Пусть $Q^{1}(x)$ и $Q^{2}(x)$ два таких многочлена. Положим

$$
\Delta(x)=Q^{1}(x)-Q^{2}(x)=\sum_{\alpha<\tau<\ell} \Delta_{\tau}(x) .
$$


Тогда $(1 / \rho(k)) \sum_{\alpha<\tau<\ell} k^{\tau}\left(\Delta_{\tau}(x), \varphi(x)\right)$ ограничена для всех $\varphi \in \mathscr{S}$, если $\alpha \neq$ $0,1, \ldots$, и для всех $\varphi \in \widetilde{\mathscr{S}}_{\left[\alpha ; P_{0}, \ldots, P_{\alpha}\right]}$, если $\alpha=0,1, \ldots$ Отсюда для тех же $\alpha$ следует, что

$$
\left(\Delta_{\tau}(x), \varphi(x)\right)=0, \quad \alpha<\tau<\ell .
$$

Из (3.44) сразу заключаем, что $\Delta_{\tau}(x) \equiv 0$, если $\alpha \neq 0,1, \ldots$ Если же $\alpha=0,1, \ldots$, то, переходя в (3.44) к преобразованию Фурье, получаем

$$
\left(\sum_{|j|=\tau} C_{j} \delta^{(j)}(t), \widetilde{\varphi}(t)\right)=0 \quad \forall \varphi \in \widetilde{\mathscr{S}}_{\left[\alpha ; P_{0}, \ldots, P_{\alpha}\right]}
$$

Полагая здесь $\widetilde{\varphi}(t)=t^{j}$ в окрестности нуля, получаем, что $C_{j}=0,|j|=\tau$, т.е. $\Delta_{\tau}(x) \equiv 0$ и в этом случае.

Нам осталось доказать лишь соотношение (3.42). Пусть $\xi \in \mathbb{R}^{n}, \eta>0$, тогда $(3.41)$ заведомо выполнено при $\varphi(x)=\omega((x-\xi) / \eta)$, поскольку $F[\omega((x-\xi) / \eta)]=$ $e^{i t \xi} \widetilde{\omega}(\eta t) \in \mathscr{S}_{\left[\ell ; P_{0}, \ldots, P_{\ell}\right]}$ для любого $\ell$. Имеем

$$
\begin{aligned}
& \frac{1}{\rho(k)}\left(G(k x), \omega\left(\frac{x-\xi}{\eta}\right)\right)=\frac{1}{\rho(k)}\left(f(k x)-Q(k x), \omega\left(\frac{x-\xi}{\eta}\right)\right) \\
& \quad=\frac{1}{\rho(k)}\left(f(k x), \omega\left(\frac{x-\xi}{\eta}\right)\right)-\frac{1}{\rho(k)} \sum_{\alpha<\tau<\ell} k^{\tau}\left(Q_{\tau}(x), \omega\left(\frac{x-\xi}{\eta}\right)\right) .
\end{aligned}
$$

Выражение слева в (3.45) по только что доказанному ограничено. Первое слагаемое справа тоже ограничено по условию (3.39), так как

$$
\frac{1}{\rho(k)}\left(f(k x), \omega\left(\frac{x-\xi}{\eta}\right)\right)=\frac{1}{\rho(k)} L_{f}^{\omega}(k \xi, k \eta) .
$$

Следовательно, ограничено и второе слагаемое справа в (3.45). Отсюда

$$
\left(Q_{\tau}(x), \omega\left(\frac{x-\xi}{\eta}\right)\right) \equiv 0, \quad \tau>\alpha .
$$

Пусть теперь в (3.42) $q=0$. Если $P_{0}=0$, то соотношение (3.42) тривиально. Если $P_{0} \neq 0$, то из $(3.46)$ вытекает

$$
0 \equiv\left(Q_{\tau}(x), \frac{1}{\eta^{n}} \omega\left(\frac{x-\xi}{\eta}\right)\right) \underset{\eta \rightarrow+0}{\longrightarrow} P_{0} Q_{\tau}(\xi) .
$$

Предположим, что (3.42) доказано для всех $q \leqslant s$. Докажем, что (3.42) справедливо и при $q=s+1$. Пусть $h(x) \in \mathscr{S}, h(x)=1$ в некоторой окрестности начала координат. В силу индуктивного предположения имеем

$$
0 \equiv\left(Q_{\tau}(x), \omega\left(\frac{x-\xi}{\eta}\right)\right)=\left(Q_{\tau}(x), \omega_{1}\left(\frac{x-\xi}{\eta}\right)\right),
$$

где $\omega_{1}(x)=\omega(x)-\sum_{q=0}^{s} P_{q}(i \partial / \partial x) h(x)$, а следовательно,

$$
\widetilde{\omega}_{1}(t)=\widetilde{\omega}(t)-\sum_{q=0}^{s} P_{q}(t) \widetilde{h}(t) .
$$


Замечая, что

$$
\widetilde{\omega}_{1}(t)=P_{a+1}(t)+o\left(|t|^{s+1}\right), \quad t \rightarrow+0
$$

имеем

$$
\begin{aligned}
& \frac{1}{\eta^{n+s+1}} \omega_{1}\left(\frac{x-\xi}{\eta}\right)=F^{-1}\left[\frac{1}{\eta^{s+1}} e^{i t \xi} \widetilde{\omega}_{1}(t \eta)\right](x) \\
& \quad=F^{-1}\left[\frac{1}{\eta^{s+1}} e^{i t \xi}\left(P_{s+1}(t)+o\left(|t|^{s+1} \eta^{s+1}\right)\right)\right](x) \underset{\eta \rightarrow+0}{\stackrel{\text { в } \mathscr{S}}{\longrightarrow}} F^{-1}\left[e^{i t \xi} P_{s+1}(t)\right](x) \\
& \quad=P_{s+1}\left(i \frac{\partial}{\partial \xi}\right) \delta(x-\xi) .
\end{aligned}
$$

Пользуясь этим, из (3.47) при $\eta \rightarrow+0$ нетрудно показать, что

$$
\begin{gathered}
\frac{1}{\eta^{n+s+1}}\left(Q_{\tau}(x), \omega_{1}\left(\frac{x-\xi}{\eta}\right)\right) \underset{\eta \rightarrow+0}{\underset{ }{\longrightarrow}}\left(Q_{\tau}(x), P_{s+1}\left(i \frac{\partial}{\partial x}\right) \delta(x-\xi)\right) \\
=(-i)^{s+1} P_{s+1}\left(i \frac{\partial}{\partial \xi}\right) Q_{\tau}(\xi)=0 .
\end{gathered}
$$

Теорема доказана.

ЗАмечаниЕ 1. Пусть в условиях теоремы сушествует еше

$$
\lim _{k \rightarrow+\infty} \frac{1}{\rho(k)} L_{f}^{\omega}(k x, k y)=C(x, y), \quad|x|^{2}+y^{2}=1, \quad y>0
$$

Тогда обобщенная функция $G(x)$, фигурирующая в теореме, обладает квазиасимптотикой на бесконечности относительно $\rho(k)$ на всех $\varphi \in \mathscr{S}$, если $\alpha \neq 0,1, \ldots$, и на всех $\varphi \in \widetilde{\mathscr{S}}_{\left[\alpha ; P_{0}, P_{1}, \ldots, P_{\alpha}\right]}$, если $\alpha=0,1, \ldots$ То есть существует

$$
\lim _{k \rightarrow+\infty} \frac{1}{\rho(k)}(G(k \xi), \varphi(\xi))=C_{\varphi} \quad \begin{cases}\forall \varphi \in \mathscr{S}, & \alpha \neq 0,1, \ldots \\ \forall \varphi \in \widetilde{\mathscr{S}}_{\left[\alpha ; P_{0}, \ldots, P_{\alpha}\right]}, & \alpha=0,1, \ldots\end{cases}
$$

Это непосредственно вытекает из доказательства теоремы, только в соответствующих местах следует использовать замечания 1 и 2 к лемме 3.1 и утверждения леммы 3.2 , стоящие в скобках.

ТЕОрема 3.3. Пусть $\mathbb{B}$ - банахово пространство, $\rho(k)$ - автомодельная функиия порядка $\alpha, f \in \mathbb{L}(\mathscr{S} \rightarrow \mathbb{B}) u \omega(x) \in \mathscr{S}$, причем $\widetilde{\omega}(t)$ не вырожсдна в нуле, а ее тейлоровское разложение в начале координат имеет вид:

$$
T_{\widetilde{\omega}}^{N}(t)=\sum_{q=0}^{N} P_{q}(t), \quad N=0,1, \ldots
$$

где $P_{q}(t)$ - однородный многочлен степени $q$ (либо тожддественный нуль). Пусть еще существуют постоянные $A$ и $b$ такие, что

$$
\sup _{k>1} \frac{1}{\rho(k)}\left\|L_{f}^{\omega}\left(\frac{x}{k}, \frac{y}{k}\right)\right\|_{\mathbb{B}} \leqslant \frac{A}{y^{b}}, \quad|x|^{2}+y^{2}=1, \quad y>0 .
$$


Тогда если $\alpha \neq 0,-1,-2, \ldots$, то существует единственный многочлен вида

$$
Q(x)=\sum_{\tau<-\alpha} Q_{\tau}(x)
$$

где $Q_{\tau}(x)$ - однородный многочлен степени $\tau$ (либо тождественный нуль), такой, что обобщенная функция $G(x)=f(x)-Q(x)$ квазиасимптотически ограничена в нуле относительно $\rho(k)$, т.е.

$$
\sup _{k>1} \frac{1}{\rho(k)}\left\|\left(G\left(\frac{x}{k}\right), \varphi(x)\right)\right\|_{\mathbb{B}}<\infty \quad \forall \varphi \in \mathscr{S} .
$$

При этом

$$
P_{q}\left(\frac{\partial}{\partial x}\right) Q_{\tau}(x)=0, \quad \tau<-\alpha, \quad q=0,1, \ldots
$$

Если же $\alpha=0,-1,-2, \ldots$, то сама $f(x)$ квазиасимптотически ограничена в нуле относительно $\rho(k)$ на всех $\varphi \in \widetilde{\mathscr{S}}_{\left[-\alpha ; P_{0}, P_{1}, \ldots, P_{-\alpha}\right]}$, т.е.

$$
\sup _{k>1} \frac{1}{\rho(k)}\left\|\left(f\left(\frac{x}{k}\right), \varphi(x)\right)\right\|_{\mathbb{B}}<\infty \quad \forall \varphi \in \widetilde{\mathscr{I}}_{\left[-\alpha ; P_{0}, \ldots, P_{-\alpha}\right]} .
$$

ДокАЗАТЕЛЬСтво. Из леммы 3.1, леммы 3.3 и замечания к ней вытекает, что если $\alpha \neq 0,-1, \ldots$, то существует многочлен вида $(3.51)$ такой, что для $G(x)=$ $f(x)-Q(x)$ справедливо неравенство (3.52), а если $\alpha=0,-1, \ldots$, то справедливо соотношение

$$
\sup _{k>1} \frac{1}{\rho(k)}\left\|\left(f\left(\frac{x}{k}\right), \varphi(x)\right)\right\|_{\mathbb{B}}<\infty \quad \forall \varphi \in \mathscr{S}, T_{\widetilde{\varphi}}^{-\alpha}(t) \equiv 0 .
$$

Чтобы в этом убедиться, достаточно в лемме 3.3 положить $g(t)=\widetilde{f}(t)$ и учесть, что

$$
F\left[\frac{1}{\rho(k)} f\left(\frac{x}{k}\right)\right]=\frac{k^{n}}{\rho(k)} \widetilde{f}(k t)
$$

Докажем, что в случае $\alpha=0,-1, \ldots$ на самом деле справедливо неравенство (3.54). Пусть $\widetilde{\varphi}(t) \in \mathscr{S}_{\left[-\alpha ; P_{0}, \ldots, P_{-\alpha}\right]}$, тогда, полагая $h(t) \in \mathscr{S}, h(t)=1$ при $|t|<1$, имеем

$$
\begin{aligned}
& \frac{1}{\rho(k)}\left(f\left(\frac{x}{k}\right), \varphi(x)\right)=\frac{k^{n}}{\rho(k)}(f(k t), \widetilde{\varphi}(t)(1-h(t))) \\
& \quad+\frac{k^{n}}{\rho(k)}\left(f(k t),\left(\widetilde{\varphi}-T_{\widetilde{\varphi}}^{-\alpha}(t)\right) h(t)\right)+\frac{k^{n}}{\rho(k)}\left(f(k t), T_{\widetilde{\varphi}}^{-\alpha}(t) h(t)\right) .
\end{aligned}
$$

Первые два слагаемых справа в этом равенстве ограничены в силу (3.55), так как тейлоровское разложение в нуле порядка $-\alpha$ соответствующих основных функций в этих слагаемых тождественно равно нулю. Третье слагаемое ограничено в силу леммы 3.1 , так как $T_{\widetilde{\varphi}}^{-\alpha}(t) h(t) \in \mathscr{S}_{\left[\ell ; P_{0}, \ldots, P_{\ell}\right]}$ для любого $\ell \geqslant-\alpha$. Что и требовалось.

Доказательство единственности многочлена (3.51), равно как и доказательство соотношений (3.53), вполне аналогично доказательству соответствуюших утверждений предыдущей теоремы. Теорема доказана. 
ЗАмЕчАнИЕ 1 . Пусть в условиях теоремы существует еше

$$
\lim _{k \rightarrow+\infty} \frac{1}{\rho(k)} L_{f}^{\omega}\left(\frac{x}{k}, \frac{y}{k}\right)=C(x, y), \quad|x|^{2}+y^{2}=1, \quad y>0 .
$$

Тогда в случае $\alpha \neq 0,-1,-2, \ldots$ обобщенная функция $G(x)$, фигурирующая в теореме, обладает квазиасимптотикой в нуле относительно $\rho(k)$, а если $\alpha=0,-1, \ldots$, то сама $f(x)$ обладает квазиасимптотикой в нуле относительно $\rho(k)$ на всех $\varphi \in$ $\widetilde{\mathscr{S}}_{\left[-\alpha ; P_{0}, P_{1}, \ldots, P_{-\alpha}\right]}$. То есть

$$
\begin{array}{lll}
\lim _{k \rightarrow+\infty} \frac{1}{\rho(k)}\left(G\left(\frac{x}{k}\right), \varphi(x)\right) & =C_{\varphi} \quad \forall \varphi \in \mathscr{S}, & \alpha \neq 0,-1, \ldots, \\
\lim _{k \rightarrow+\infty} \frac{1}{\rho(k)}\left(f\left(\frac{x}{k}\right), \varphi(x)\right) & =C_{\varphi} \quad \forall \varphi \in \widetilde{\mathscr{S}}_{\left[-\alpha ; P_{0}, \ldots, P_{-\alpha}\right]}, & \alpha=0,-1, \ldots
\end{array}
$$

Отметим, что в теоремах 3.2 и 3.3 некоторые слагаемые, составляющие многочлен $Q(x)$, фигурирующий в формулах (3.40) и (3.51), могут отсуствовать, если $\varphi$ подчинено некоторьм добавочньм условиям. А именно, имеет место следующее

ЗАМЕчАНИЕ 2 К ТЕОРЕМАМ 3.2 и 3.3. Пусть в условиях теоремы 3.2 (теоремы 3.3) $\varphi(x) \in \mathscr{S}$ такова, что тейлоровское разложение ее преобразования Фурье в нуле $T_{\widetilde{\varphi}}^{N}(t)=\sum_{q=0}^{N} \Phi_{q}(t)$, где $\Phi_{q}(t)$ - однородньй многочлен степени $q$, содержит слагаемое

$$
\Phi_{q_{0}}(t) \in \mathbb{P}\left[P_{0}, P_{1}, \ldots, P_{q_{0}}\right]
$$

Тогда

$$
\left(Q_{q_{0}}(k \xi), \varphi(\xi)\right)=0 .
$$

Действительно, переходя к преобразованию Фурье (считая $N>q_{0}$ ), полагая $h(x) \in \mathscr{S}$ такой, что $\widetilde{h}(t)=1$ в некоторой окрестности нуля, имеем

$$
\begin{gathered}
\left(Q_{q_{0}}(i \xi), \varphi(\xi)\right)=\left(Q_{q_{0}}\left(\frac{\partial}{\partial t}\right) \delta(t), \widetilde{\varphi}(t)\right)=\left(Q_{q_{0}}\left(\frac{\partial}{\partial t}\right) \delta(t),\left(\widetilde{\varphi}(t)-T_{\widetilde{\varphi}}^{N}(t)\right) \widetilde{h}(t)\right) \\
+\left(Q_{q_{0}}\left(\frac{\partial}{\partial t}\right) \delta(t), T_{\widetilde{\varphi}}^{N}(t) \widetilde{h}(t)\right)=\left(Q_{q_{0}}\left(\frac{\partial}{\partial t}\right) \delta(t), \Phi_{q_{0}}(t) \widetilde{h}(t)\right) \\
=\left(Q_{q_{0}}(\xi), \Phi_{q_{0}}\left(-i \frac{\partial}{\partial \xi}\right) h(\xi)\right)=\left(\Phi_{q_{0}}\left(i \frac{\partial}{\partial \xi}\right) Q_{q_{0}}(\xi), h(\xi)\right)=0 .
\end{gathered}
$$

В последнем равенстве мы учли, что $\Phi_{q_{0}}$ разлагается в сумму, в каждом из слагаемых которой присуствуют множителями $P_{q}$. А для них согласно (3.42) (или (3.53) в случае теоремы 3.3$) P_{q}(\partial / \partial \xi) Q_{q_{0}}(\xi)=0$.

СлЕДСТВИЕ. Пусть в условиях теоремы 3.3 (теоремы 3.2) функиия $\varphi(x) \in$ $\widetilde{\mathscr{S}}_{\left[d ; P_{0}, \ldots, P_{d}\right]}$ для некоторого $d=0,1, \ldots$ Тогда в $(3.51)$ (соответственно в (3.40)) можсно считать, что $Q(x)=\sum_{d<\tau<-\alpha} Q_{\tau}(x)$ (соответственно $\left.Q(x)=\sum_{\max \{d, \alpha\}<\tau<\ell} Q_{\tau}(x)\right)$.

$B$ частности, если $d=[-\alpha]$, то в $(3.51)$ многочлен $Q(x) \equiv 0$, т.е. соотношение (3.52) перепишется в виде

$$
\sup _{k>1} \frac{1}{\rho(k)}\left\|\left(f\left(\frac{x}{k}\right), \varphi(x)\right)\right\|_{\mathbb{B}}<\infty \quad \forall \varphi \in \widetilde{\mathscr{S}}_{\left[[-\alpha] ; P_{0}, \ldots, P_{[-\alpha]}\right]} .
$$


ЗАМЕчАНИЕ 3. Предположим, что $\operatorname{supp} \tilde{f} \subset$ Г. В этом случае все предыдушие рассмотрения (в том числе теоремы 3.2 и 3.3 ) останутся справедливыми, если условие невырожденности в нуле $\widetilde{\omega}(t)$ заменить более слабым.

А именно, сушествует $m$ такое, что тейлоровское разложение $\widetilde{\omega}(t)$ в начале координат удовлетворяет следуюшему условию: для любого вектора $\bar{e} \in \Gamma,|\bar{e}|=1$,

$$
T_{\varphi}^{m}(\lambda \bar{e}) \not \equiv 0, \quad \lambda>0 .
$$

Все доказательства проводятся по той же самой схеме, при этом следует пользоваться вариантом леммы П.1, отмеченном в замечании 3 к этой лемме.

\section{§4. Некоторые применения}

В этом параграфе полученные вьше теоремы применяются для изучения асимптотических свойств решений задачи Коши для уравнения теплопроводности, задачи Коши для диффузии многокомпонентных вешеств и некомпенсации граничных особенностей голоморфных функций в трубчатых областях (в одномерном случае cм. [1]).

4.1. Задача Коши для уравнения теплопроводности. Рассмотрим задачу Коши

$$
\begin{gathered}
\frac{\partial}{\partial t} u(x, t)=a^{2} \Delta u(x, t), \quad \lim _{t \rightarrow+0} u(x, t)=f(x) \quad\left(\mathrm{в} \mathscr{S}^{\prime}\right), \\
f \in \mathscr{S}^{\prime}, \quad x \in \mathbb{R}^{n}, \quad t>0,
\end{gathered}
$$

в классе не более чем полиномиально растущих функций, т.е. таких, что

$$
|u(x, t)|<C \frac{1}{t^{b}}\left(|x|^{N}+|t|^{N}+1\right)
$$

для некоторых $b, C$ и $N$, зависящих от $u$. Как известно, в этом классе существует единственное решение задачи (4.1), определяемое формулой

$$
u(x, t)=\left(f(\xi), \frac{1}{(2 a \sqrt{\pi t})^{n}} e^{-(x-\xi)^{2} /\left(4 a^{2} t\right)}\right) \equiv L_{f}^{\omega}(x, y)
$$

где

$$
\omega(\xi)=\frac{1}{(2 a \sqrt{\pi})^{n}} e^{-\xi^{2} /\left(4 a^{2}\right)} \quad\left(\widetilde{\omega}(t)=e^{-a^{2} t^{2}}\right), \quad y=\sqrt{t} .
$$

Пусть $\rho(k)$ - автомодельная функция порядка $\alpha$. Если для некоторой функции $u(x, t)$ выполнены условия:

1) существует предел

$$
\frac{1}{\rho(k)} u\left(k x, k^{2} t\right) \underset{k \rightarrow+\infty}{\longrightarrow} u_{0}(x, t), \quad x \in \mathbb{R}^{n}, \quad t>0 ;
$$

2) сушествуют $A$ и $b$ такие, что

$$
\left|\frac{1}{\rho(k)} u\left(k x, k^{2} t\right)\right| \leqslant \frac{A}{t^{b}}, \quad|x|^{2}+t^{2}=1,
$$

то будем говорить, что функция $u(x, t)$ стабилизируется (в бесконечности) по параболам относительно автомодельной функиии $\rho(k)$. 
ТЕОРема 4.1. Пусть $\rho(k)$ - автомодельная функиия. Для того чтобь решение задачи Коши (4.1) стабилизировалось по параболам относительно $\rho(k)$, необходимо и достаточно, чтобь начальная функиия $f(x) \in \mathscr{S}^{\prime}$ обладала квазиасимптотикой на бесконечности относительно $\rho(k)$, т.е.

$$
\frac{1}{\rho(k)}(f(k \xi), \varphi(\xi)) \underset{k \rightarrow+\infty}{\longrightarrow} c_{\varphi} \quad \forall \varphi \in \mathscr{S}
$$

ДокАЗАТЕльство. Пусть выполнено (4.6). Тогда (4.4) и (4.5) следуют из замечания к теореме $3.1^{\prime}$ при $P_{0} \neq 0$, если учесть формулы (4.2) и (4.3).

Обратно. Пусть решение задачи Коши (4.1) $u(x, t)$ удовлетворяет условиям (4.4) и (4.5). Заметим сначала, что

$$
\frac{1}{\rho(k)} u\left(k x, k^{2} t\right)=\frac{1}{\rho(k)} L_{f}^{\omega}(k x, k y)
$$

где $\omega$ и $y$ определяются по формуле (4.3). Из (4.4) и (4.5) следует, что

$\lim _{k \rightarrow+\infty} \frac{1}{\rho(k)} L_{f}^{\omega}(k x, k y)=C(x, y), \quad \frac{1}{\rho(k)}\left|L_{f}^{\omega}(k x, k y)\right| \leqslant \frac{A}{y^{b}}, \quad|x|^{2}+y^{2}=1, \quad y>0$.

Пользуясь теперь теоремой 3.2 (точнее, замечанием к ней) и учитьвая, что $P_{0} \neq 0$, а, стало быть, в силу (3.42) $Q(x) \equiv 0$, получаем соотношение (4.6). Теорема доказана.

СлЕДСТВИЕ. Пусть решение задачи Коши (4.1) и $(x, t)$ стабилизируется по параболам относительно автомодельной функиии $\rho(k)$. Тогда оно стабилизируется по времени относительно функиии $\rho(\sqrt{t})$, m.е. для любого $x \in \mathbb{R}^{n}$ существует

$$
\lim _{t \rightarrow+\infty} \frac{1}{\rho(k)} u(x, t)=u_{0}
$$

Действительно,

$$
\begin{aligned}
\frac{1}{\rho(\sqrt{t})} u(x, t) & =\frac{1}{\rho(y)} L_{f}^{\omega}(x, y)=\frac{1}{\rho(y)}\left(f(\xi), \frac{1}{y^{n}} \omega\left(\frac{x-\xi}{y}\right)\right) \\
& =\frac{1}{\rho(y)}\left(f(y \xi), \omega\left(\frac{x}{y}-\xi\right)\right) \underset{y \rightarrow+\infty}{\longrightarrow}\left(f_{0}(\xi), \omega(-\xi)\right)=c_{\omega}
\end{aligned}
$$

Здесь мы воспользовались тем, что согласно теореме 4.1 начальная функция $f(x)$ задачи Коши (4.1) обладает квазиасимптотикой на бесконечности относительно $\rho(k)$, и тем, что $\omega(x / y-\xi) \rightarrow \omega(-\xi)$ в $\mathscr{S}$ при любых $x \in \mathbb{R}^{n}$ и $y \rightarrow+\infty$.

4.2. Задача Коши для диффузии многокомпонентных веществ. Рассмотрим следуюшую модельную задачу:

$$
\begin{array}{rlrl}
\frac{\partial}{\partial t} u_{i}(x, t) & =a_{i}^{2} \Delta u_{i}(x, t), & i & =1,2, \ldots, m \\
\lim _{t \rightarrow+0} u_{i}(x, t) & =f(x) \quad(\text { в } \mathscr{S}), & f \in \mathscr{S}
\end{array}
$$


Здесь $u_{i}(x, t)$ - концентрация $i$-го вешества, $i=1,2, \ldots, m$. Не нарушая общности, можно считать, что все коэффициенты диффузии различных веществ $a_{i}$ различны. Рассмотрим линейную комбинацию

$$
U(x, t)=\sum_{i=1}^{m} c_{i} u_{i}(x, t)
$$

Ее можно интерпретировать, например, как плотность зарядов смеси веществ, где $c_{i}$ - удельный заряд $i$-ой компоненты.

Пусть $U(x, t)$ стабилизируется относительно $\rho(k)$ по параболам. Что можно сказать об асимптотическом поведении концентрации отдельных веществ $u_{i}$ ? Положим

$$
s_{p}=\sum_{i=1}^{m} c_{i} a_{i}^{2 p}, \quad p=0,1, \ldots
$$

ТЕОРема 4.2. Пусть $U(x, t)$ стабилизируется по параболам относительно автомодельной функиии $\rho(k)$ порядка $\alpha$. Тогда если $s_{0}=\sum_{i=1}^{m} c_{i} \neq 0$, то каждая компонента $u_{i}(x, t)$ стабилизируется по параболам относительно функиии $\rho(k)$.

Eсли жсе

$$
s_{p}=0, \quad p=0,1, \ldots, M-1, \quad s_{M} \neq 0 \quad(M>0), \quad \alpha \neq 0,1, \ldots,
$$

то существует $M$-гармонический многочлен $Q(x), \Delta^{M} Q(x)=0$, такой, что для каждого $i=1, \ldots$, m функиия

$$
\widehat{u}_{i}(x, t)=u_{i}(x, t)-\sum_{q=0}^{M-1} \frac{\left(a_{i}^{2} t\right)^{q}}{q !} \Delta^{q} Q(x)
$$

стабилизируется по параболам относительно $\rho(k)$.

ДокАЗАтЕльство. Заметим, что

$$
\begin{aligned}
& u_{i}\left(k x, k^{2} t\right)=L_{f}^{\omega_{i}}(k x, k y), \quad \omega_{i}(x)=\frac{1}{\left(2 a_{i} \sqrt{\pi}\right)^{n}} e^{-x^{2} /\left(4 a_{i}^{2}\right)}, \quad y=\sqrt{t}, \\
& U\left(k x, k^{2} t\right)=L_{f}^{\omega}(k x, k y), \quad \omega(x)=\sum_{i=1}^{m} c_{i} \omega_{i}(x) .
\end{aligned}
$$

По условию

$\lim _{k \rightarrow+\infty} \frac{1}{\rho(k)} L_{f}^{\omega}(k x, k y)=C(x, y), \quad \frac{1}{\rho(k)}\left|L_{f}^{\omega}(k x, k y)\right| \leqslant \frac{A}{y^{b}}, \quad|x|^{2}+y^{2}=1, \quad y>0$ для некоторых $A$ и $b$. Воспользуемся теперь теоремой 3.2 (точнее, замечанием к ней). Если $s_{0} \neq 0$, то $P_{0} \neq 0$. Следовательно, $f(x)$ обладает квазиасимптотикой на бесконечности относительно $\rho(k)$, а потому согласно замечанию к теореме $3.1^{\prime}$ при любом $i=1, \ldots, m u_{i}(x, t)$ стабилизируется по параболам относительно $\rho(k)$.

Пусть теперь $s_{p}=0, p=0,1, \ldots, M-1, s_{M} \neq 0$ и $\alpha \neq 0,1, \ldots$. Тогда, учитывая, что $\widetilde{\omega}(t)=\sum_{i=1}^{m} c_{i} e^{-a_{i}^{2} t}$, имеем $P_{q} \equiv 0$ при $q=0,1, \ldots, M-1$, а $P_{M}(t)=(-1)^{M} /(M !) s_{M}|t|^{2 M}$. Согласно замечанию 1 к теореме 3.2 существует 
многочлен $Q(x)$ такой, что $\Delta^{M} Q(x)=0$ и функция $G(x)=f(x)-Q(x)$ обладает квазиасимптотикой на бесконечности относительно $\rho(k)$. Далее, замечая, что функция $\widehat{u}_{i}(x, t)($ см. $(4.10))$ является решением задачи Коши

$$
\frac{\partial}{\partial t} u_{i}(x, t)=a_{i}^{2} \Delta u_{i}(x, t), \quad \widehat{u}_{i}(x, 0)=G(x), \quad i=1,2, \ldots, m
$$

по теореме 4.1 заключаем, что $\widehat{u}_{i}$ стабилизируется по параболам относительно $\rho(k)$. Теорема доказана.

СЛЕДСТВИЕ. Пусть $U(x, t)$ стабилизируется по параболам относительно автомодельной функиии $\rho(k)$ порядка $\alpha \neq 0,1, \ldots u s_{p}=0$ при $p=$ $0,1, \ldots, M-1$, a $s_{M} \neq 0$. Пусть еще $\alpha>2 M-2$. Тогда кажсдая компонента $u_{i}(x, t), i=1, \ldots, m$, стабилизируется по времени относительно $\rho(\sqrt{t})$.

Действительно, по теореме $\widehat{u}_{i}(x, t)$ стабилизируется по параболам относительно автомодельной функции $\rho(k)$. Следовательно, согласно следствию к теореме 4.1 она стабилизируется по времени относительно $\rho(\sqrt{t})$. Теперь наше утверждение вытекает из того факта, что при $\alpha>2 M-2$

$$
\frac{1}{\rho(\sqrt{t})} \sum_{q=0}^{M-1} \frac{\left(a_{i}^{2} t\right)^{q}}{q !} \Delta^{q} Q(x) \underset{t \rightarrow+\infty}{\longrightarrow} 0 .
$$

4.3. О некомпенсации граничных особенностей голоморфных функций в трубчатых областях над острыми конусами. Пусть $F(z) \in H\left(T^{C}\right)$, где $C$ - острый выпуклый открытый конус в $\mathbb{R}^{n}$, а $T^{C}=\mathbb{R}^{n}+i C$ - трубчатая область над конусом $C$. Это означает, что $F(z)$ голоморфна в $T^{C}$ и удовлетворяет там оценке

$$
|F(z)| \leqslant A \frac{(1+|z|)^{d}}{\Delta_{C}^{b}(y)}, \quad z=x+i y, \quad y \in C
$$

с некоторыми постоянными $A, b$ и $d$. Здесь $\Delta_{C}(y)$ - расстояние от $y \in C$ до границы конуса $C$. Отметим, что у $F(z)$ существует граничное значение в $\mathscr{S}^{\prime}$ при $y \rightarrow+0$ такое, что $f(x)=\lim _{y \rightarrow+0} F(x+i y)$, причем носитель его преобразования Фурье лежит в конусе, сопряженном к конусу $C$, т.е.

$$
\operatorname{supp} \tilde{f}(t) \subset \Gamma=C^{*}
$$

(см. [2]). Отметим, что для любого вектора $y \in C$

$$
F(x+i y)=\left(\tilde{f}(t), e^{i(x, t)} e^{-(y, t)}\right)=\left(f(\xi), \omega_{y}(x-\xi)\right)=L_{f}^{\omega_{y}}(x, 1),
$$

где $\omega_{y}(x) \in \mathscr{S}$ такова, что $\widetilde{\omega}_{y}(t)=e^{-(y, t)}$ при $t \in \Gamma$, а вне $Г$ она является каким-либо продолжением этой экспоненты до основной функции из $\mathscr{S}$.

Пусть заданы автомодельная функция $\rho(k)$, векторы $h_{1}$ и $h_{2}$ из $C$, числа $c_{1}$ и $c_{2}$ и выполнена оценка

$$
\frac{1}{\rho(k)}\left|c_{1} F\left(\frac{x+i h_{1}}{k}\right)+c_{2} F\left(\frac{x+i h_{2}}{k}\right)\right| \leqslant A(1+|x|)^{a}, \quad x \in \mathbb{R}^{n}, \quad k>1,
$$

с некоторыми постоянньми $A$ и $а$. Нас будет интересовать вопрос, что можно сказать о поведении самой функции $F(z)$ в окрестности начала координат? 
ТЕОРема 4.3. Пусть $F(z) \in H\left(T^{C}\right)$ и удовлетворяет оценке $(4.14)$ с некоторой автомодельной функичей $\rho(k)$ порядка $\alpha$, причем $\widehat{h}=h_{1}-h_{2} \in C$. Тогда

1) если $c_{1}+c_{2} \neq 0$, то существуют постоянные $A_{1}, a_{1}$ и $b_{1}$ такие, что

$$
\sup _{k>1} \frac{1}{\rho(k)}\left|F\left(\frac{z}{k}\right)\right| \leqslant A_{1} \frac{(1+|x|)^{a_{1}}}{\Delta_{C}^{b_{1}}(y)}, \quad z \in T^{C}
$$

2) если $c_{1}+c_{2}=0$ (не нарушая общности, можнно считать, что $c_{1}=$ $\left.-c_{2}=1\right)$ и, кроме того,

a) если $\alpha>0$, то выполняется соотношение (4.15);

b) если $\alpha<0$ и $\alpha \neq-1,-2, \ldots$, то существует многочлен $Q(z)$ степени меньшей $-\alpha$, удовлетворяющий уравнению

$$
\left(\widehat{h}, \frac{\partial}{\partial z}\right) Q(z)=0
$$

такой, что

$$
\sup _{k>1} \frac{1}{\rho(k)}\left|F\left(\frac{z}{k}\right)-Q\left(\frac{z}{k}\right)\right| \leqslant A_{1} \frac{(1+|x|)^{a_{1}}}{\Delta_{C}^{b_{1}}(y)}, \quad z \in T^{C},
$$

для некоторых постоянных $A_{1}, a_{1} u b_{1}$.

ДокАЗАТЕЛЬСТво. Из соотношения (4.13) вытекает, что

$$
c_{1} F\left(\frac{x+i h_{1}}{k}\right)+c_{2} F\left(\frac{x+i h_{2}}{k}\right)=L_{f}^{\omega}\left(\frac{x}{k}, \frac{1}{k}\right), \quad k>1,
$$

где $f(x)$ есть граничное значение $F(z)$, а $\omega(x) \in \mathscr{S}$ и $\widetilde{\omega}(t)=c_{1} e^{-\left(h_{1}, t\right)}+c_{2} e^{-\left(h_{2}, t\right)}$, $t \in \Gamma$. Из условия (4.14) и замечания 2 к лемме П.4 следует, что

$$
\sup _{k>1} \frac{1}{\rho(k)}\left|L_{f}^{\omega}\left(\frac{x}{k}, \frac{\varkappa}{k}\right)\right| \leqslant \frac{A_{1}(1+|x|)^{a_{1}}}{\varkappa^{b}}, \quad|x|^{2}+\varkappa^{2}=1, \quad \varkappa>0 .
$$

Заметим, что $T_{\widetilde{\omega}}^{1}(t)=\left(c_{1}+c_{2}\right)-\left(\left(c_{1} h_{1}+c_{2} h_{2}\right), t\right)=P_{0}+P_{1}(t)$.

Пусть $c_{1}+c_{2} \neq 0$. Тогда $\widetilde{\omega}(t)$ не вырождена в нуле и $P_{0} \neq 0$, а потому по теореме 3.3 заключаем, что $f(x)$ квазиасимптотически ограничена в нуле относительно $\rho(k)$. Следовательно, $\widetilde{f}(t)$ квазиасимптотически ограничена на бесконечности относительно $\left(1 / k^{n}\right) \rho(k)$. Теперь оценка (4.15) следует из того, что

$$
\begin{aligned}
\frac{1}{\rho(k)}\left|F\left(\frac{z}{k}\right)\right| & =\frac{k^{n}}{\rho(k)}\left|\left(\tilde{f}(k t), e^{i(x, t)-(y, t)}\right)\right| \\
& \leqslant C \max _{|j| \leqslant N} \sup _{t \in \Gamma}(1+|t|)^{N}\left|\frac{\partial^{|j|}}{\partial t^{j}} e^{i(x, t)-(y, t)}\right|
\end{aligned}
$$

для некоторых $C$ и $N$.

Пусть теперь $c_{1}=-c_{2}=1$. Тогда $P_{0}=0$, a $P_{1}(t)=(\widehat{h}, t) \neq 0$ при $t \in \Gamma$, и мы снова можем воспользоваться теоремой 3.3 (см. замечание 3 к ней). Из этой 
теоремы следует, что если $\alpha>0$, то по-прежнему $f(x)$ квазиасимптотически ограничена в нуле относительно $\rho(k)$ и оценка (4.15) получается так же, как и в предыдущем случае. Если же $\alpha<0$ и $\alpha \neq-1,-2, \ldots$, то сушествует многочлен $Q(z)=\sum_{0 \leqslant \tau<-\alpha} Q_{\tau}(z)$ такой, что

$$
P_{1}\left(\frac{\partial}{\partial x}\right) Q(x)=\left(\widehat{h}, \frac{\partial}{\partial x}\right) Q(x)=0
$$

и функция $G(x)=f(x)-Q(x)$ квазиасимптотически ограничена в нуле относительно $\rho(k)$. Переходя к преобразованию Фурье и действуя, как и в предыдущем случае, получаем требуемую оценку (4.17). Теорема доказана.

ЗАмЕчАниЕ 1. Если наряду с оценкой (4.14) потребовать еще существование пределов

$$
\lim _{k \rightarrow+\infty} \frac{1}{\rho(k)}\left(c_{1} F\left(\frac{x+i h_{1}}{k}\right)+c_{2} F\left(\frac{x+i h_{2}}{k}\right)\right)=C(x), \quad x \in \mathbb{R}^{n}
$$

то можно утверждать, что наряду с оценками (4.15) и (4.17) сушествуют еще пределы

$$
\lim _{k \rightarrow+\infty} \frac{1}{\rho(k)} F\left(\frac{z}{k}\right)=C(x, y), \quad z \in T^{C}
$$

и

$$
\lim _{k \rightarrow+\infty} \frac{1}{\rho(k)}\left(F\left(\frac{z}{k}\right)-Q\left(\frac{z}{k}\right)\right)=C(x, y), \quad z \in T^{C},
$$

соответственно.

Доказательство проводится аналогично, следует только воспользоваться замечанием 1 к теореме 3.3 .

ЗАмЕчАниЕ 2. В условиях теоремы и существования предела (4.18) при $\alpha<0$ и $\alpha \neq-1,-2, \ldots$ сушествуют постоянные $Q_{0}$ и $C$ такие, что

$$
\lim _{k \rightarrow+\infty} \frac{1}{\rho(k)}\left(F\left(\frac{\zeta \widehat{h}}{k}\right)-Q_{0}\right)=C(\zeta)^{-\alpha}, \quad \zeta \in \mathbb{C}, \quad \operatorname{Im} \zeta>0
$$

Действительно, нетрудно заметить, что в силу (4.16)

$$
\frac{d}{d \zeta} Q(\zeta \widehat{h})=\left.\left(\widehat{h}, \frac{\partial}{\partial x}\right) Q(x)\right|_{x=\zeta \widehat{h}}=0, \quad \zeta \in \mathbb{C}, \quad \operatorname{Im} \zeta>0
$$

и, следовательно, $Q(\zeta \widehat{h} / k)=Q(0)=Q_{0}$. Согласно теореме 3.3 и предыдушему замечанию

$$
\frac{1}{\rho(k)}\left(F\left(\frac{\zeta \widehat{h}}{k}\right)-Q_{0}\right) \underset{k \rightarrow+\infty}{\longrightarrow} c(\zeta) .
$$

Ясно, что $c(\zeta)$ - голоморфная в верхней полуплоскости функция, однородная степени $-\alpha$, а потому $c(\zeta)=C(\zeta)^{-\alpha}$. 


\section{§5. Приложение}

В этом приложении мы докажем ряд вспомагательных утверждений, которые были использованы в основном тексте.

Следующая лемма является некоторым вариантом классической теоремы о короне. Мы приводим ее в том виде, который нам был нужен в основном тексте (ср. также [5]).

ЛЕмма П.1. Пусть $\mathbb{J}$ - произвольное множество индексов и задано семейство функиий $\left\{\eta_{\beta}(t) \in C^{\infty}\left(\mathbb{R}^{n}\right), \beta \in \mathbb{J}\right\}$ такое, что существуют числа

$$
q, Q_{j}, C_{l} \quad u \quad c>0, \quad j=\left(j_{1}, \ldots, j_{n}\right), \quad j_{k}=0,1, \ldots, \quad k=1,2, \ldots, n,
$$

такие, что для любого мультиндекса $ј$ выполнены оценки

$$
\begin{array}{ll}
\left|\frac{\partial^{|j|}}{\partial t^{j}} \eta_{\beta}(t)\right| \leqslant C_{j}(1+|t|)^{Q_{j}}, & \beta \in \mathbb{J}, \quad t \in \mathbb{R}^{n}, \\
\sup _{\beta \in \mathbb{J}}\left|\eta_{\beta}(t)\right| \geqslant \frac{c}{(1+|t|)^{q}}, & t \in \mathbb{R}^{n} .
\end{array}
$$

Тогда существуют счетное множество индексов $I \subset \mathbb{J}$, иисла $\lambda_{i}>0, \beta_{i} \in I$, $i=1,2, \ldots, \sum_{i=1}^{\infty} \lambda_{i}=1$, и функции $\left\{g_{i}(t) \in C^{\infty}\left(\mathbb{R}^{n}\right), i=1,2, \ldots\right\}$ такие, что для любого мультиндекса $j$ найдутся постоянные $C_{j}^{*}$ и $Q_{j}^{*}$ такие, что

$$
\left|\frac{\partial^{|j|}}{\partial t^{j}} g_{i}(t)\right| \leqslant C_{j}^{*}(1+|t|)^{Q_{j}^{*}}, \quad t \in \mathbb{R}^{n}, \quad i=1,2, \ldots,
$$

и выполнено тохсдество

$$
\sum_{i=1}^{\infty} \lambda_{i} g_{i}(t) \eta_{\beta_{i}}(t)=1, \quad t \in \mathbb{R}^{n}
$$

ДоказАТЕльство. Сначала докажем, что существуют счетное множество индексов $I \subset \mathbb{J}$, числа $\lambda_{i}>0, \beta_{i} \in I, i=1,2, \ldots, \sum_{i=1}^{\infty} \lambda_{i}=1$, постоянные $d$ и $\ell$ такие, что

$$
\sum_{i=1}^{\infty} \lambda_{i}\left|\eta_{\beta_{i}}(t)\right|^{2} \geqslant \frac{d}{(1+|t|)^{\ell}}, \quad t \in \mathbb{R}^{n} .
$$

Для этого положим

$$
r_{\beta}(t)=\min \left\{1, \frac{1}{2 n C_{1}(2+|t|)^{Q_{1}}}\left|\eta_{\beta}(t)\right|\right\}, \quad \beta \in \mathbb{J}, \quad t \in \mathbb{R}^{n},
$$

где $C_{1}=\max _{|j|=1}\left\{C_{j}\right\}$ и $Q_{1}=\max _{|j|=1}\left\{Q_{j}\right\}$. Покажем, что

$$
\left|\eta_{\beta}(t)\right| \geqslant \frac{1}{2}\left|\eta_{\beta}\left(t^{0}\right)\right|, \quad\left|t-t^{0}\right| \leqslant r_{\beta}\left(t^{0}\right) .
$$


Действительно, полагая $\Phi_{\beta}(\xi)=\eta_{\beta}\left(t^{0}+\xi\left(t-t^{0}\right)\right)$, по лемме о конечных приращениях имеем

$$
\begin{aligned}
& \left|\eta_{\beta}(t)-\eta_{\beta}\left(t^{0}\right)\right|=\left|\Phi_{\beta}(1)-\Phi_{\beta}(0)\right|=\left|\Phi_{\beta}^{\prime}\left(\xi^{*}\right)\right| \\
& \quad=\left|\sum_{k=1}^{n} \frac{\partial \eta_{\beta}\left(t+\xi^{*}\left(t-t^{0}\right)\right)}{\partial t_{k}}\left(t_{k}-t_{k}^{0}\right)\right| \leqslant\left|t-t^{0}\right| \sum_{k=1}^{n}\left|\frac{\partial \eta_{\beta}\left(t^{*}\right)}{\partial t_{k}}\right| \\
& \quad \leqslant n r_{\beta}\left(t^{0}\right) C_{1}\left(1+1+\left|t^{0}\right|\right)^{Q_{1}} \leqslant \frac{1}{2}\left|\eta_{\beta}\left(t^{0}\right)\right| .
\end{aligned}
$$

Мы учли соотношение (П.6)

$\mathrm{B}$ силу (П.2) для каждой точки $t \in \mathbb{R}^{n}$ найдется $\beta(t) \in \mathbb{J}$ такое, что

$$
\left|\eta_{\beta(t)}(t)\right| \geqslant \frac{1}{2} \frac{c}{(1+|t|)^{q}}
$$

в частности, $r_{\beta\left(t^{0}\right)}\left(t^{0}\right)>0$. Поэтому

$$
\left|\eta_{\beta\left(t^{0}\right)}(t)\right| \geqslant \frac{1}{2}\left|\eta_{\beta\left(t^{0}\right)}\left(t^{0}\right)\right| \geqslant \frac{1}{4} \frac{c}{\left(1+\left|t^{0}\right|\right)^{q}}, \quad\left|t-t^{0}\right| \leqslant r_{\beta\left(t^{0}\right)}\left(t^{0}\right) .
$$

Положим $T_{p}=\{|t|<p\}$ и $K_{p}=\overline{T_{p} \backslash T_{p-1}}$. Если $t^{0} \in K_{p}$, то из (П.6) и (П.8) следует, что

$$
r_{\beta\left(t^{0}\right)}\left(t^{0}\right) \geqslant \min \left\{1, \frac{c}{8 n C_{1}\left(1+\left|t^{0}\right|\right)^{Q_{1}+q}}\right\} \geqslant \min \left\{1, \frac{c}{8 C_{1} n(1+p)^{Q_{1}+q}}\right\}
$$

(мы учли, что $p-1 \leqslant\left|t^{0}\right| \leqslant p$ ). Таким образом, каждый $K_{p}$ покрыт шарами радиуса $r_{\beta(t)}(t)$ и из этого покрытия выделим покрытие конечной кратности $N$ (см. [6]), где $N$ зависит только от размерности пространства $n$. Центры выбранных шаров перенумеруем следуюшим образом. Сначала нумеруем шары, покрываюшие $K_{1}$, затем $K_{2}$ и т.д. Получим счетное семейство шаров, покрывающее все $\mathbb{R}^{n}$. Их центры обозначим через $t^{i}$, а сами шары - через $S\left(t^{i}\right)$. Объем такого шара не менше чем $\sigma_{n}\left(r_{\beta\left(t^{i}\right)}\left(t^{i}\right)\right)^{n}$ (здесь $\sigma_{n}=\pi^{n / 2} / \Gamma(n / 2+1)$ - объем еденичного шара в $\mathbb{R}^{n}$ ). Поскольку

$$
\sum_{t^{i} \in K_{p}} \sigma_{n}\left(r_{\beta\left(t^{i}\right)}\left(t^{i}\right)\right)^{n} \leqslant N \cdot\left[\text { объем } T_{p+1}\right] \leqslant N \sigma_{n}(p+1)^{n},
$$

то количество выбранных шаров $M_{p}$, покрывающих $K_{p}$, удовлетворяет соотношению

$$
M_{p} \leqslant N(p+1)^{n}\left(\frac{8 n C_{1}(1+p)^{Q_{1}+q}}{c}\right)^{n} .
$$

Положим теперь $\lambda_{i}=6 /\left(\pi^{2} i^{2}\right)$, тогда $\sum_{i=1}^{\infty} \lambda_{i}=1$. Обозначим $\beta_{i}=\beta\left(t^{i}\right)$, a через $I$ - счетное множество $\left\{\beta_{i}, i=1,2, \ldots\right\}$.

Пусть теперь точка $t \in K_{p}$, тогда она обязательно попадет в какой-то шар $S\left(t^{i_{0}}\right)$, покрываюший $K_{p}$. Имеем

$$
\sum_{i=1}^{\infty} \lambda_{i}\left|\eta_{\beta_{i}}(t)\right|^{2} \geqslant \lambda_{i_{0}}\left|\eta_{\beta_{i_{0}}}(t)\right|^{2}
$$


Из всей суммы мы оставили только то слагаемое, которое соответствует шару $S\left(t^{i_{0}}\right)$. Согласно (П.8) имеем

$$
\lambda_{i_{0}}\left|\eta_{\beta_{i_{0}}}(t)\right|^{2} \geqslant \lambda_{i_{0}}\left(\frac{1}{4} \frac{c}{\left(1+\left|t^{i_{0}}\right|\right)^{q}}\right)^{2} \geqslant \lambda_{i_{0}} \frac{c^{2}}{16(1+p)^{2 q}}
$$

Оценим $\lambda_{i_{0}}$, учитьвая, что $i_{0}$ - это номер одного из шаров, покрывающих $K_{p}$. В силу (П.10)

$$
\begin{aligned}
i_{0} & \leqslant M_{1}+M_{2}+\cdots+M_{p} \leqslant \sum_{\tau=1}^{p} N\left(\frac{8 n C_{1}}{c}\right)^{n}(1+\tau)^{\left(Q_{1}+q+1\right) n} \\
& \leqslant N\left(\frac{8 C_{1} n}{c}\right)^{n}(p+1)^{n\left(Q_{1}+q+1\right)+1}
\end{aligned}
$$

Отсюда

$$
\lambda_{i_{0}}=\frac{6}{\pi^{2} i_{0}^{2}} \geqslant \frac{6 c^{2 n}}{\pi^{2} N^{2} 64^{n} n^{2 n} C_{1}^{2 n}} \frac{1}{(p+1)^{2 n\left(1+Q_{1}+q\right)+2}} .
$$

Теперь из (П.11) и (П.12) имеем

$$
\begin{aligned}
\sum_{i=1}^{\infty} \lambda_{i}\left|\eta_{\beta_{i}}(t)\right|^{2} & \geqslant \frac{6 c^{2 n+2}}{16^{n+1} \pi^{2} N^{2} n^{2 n} C_{1}^{2 n}} \frac{1}{(p+1)^{2 n\left(1+Q_{1}+q\right)+2 q+2}} \\
& \geqslant \frac{\mathrm{const}}{(2+|t|)^{2 n\left(1+Q_{1}+q\right)+2 q+2}}
\end{aligned}
$$

(здесь учтено, что $p-1 \leqslant|t| \leqslant p)$. Из (П.13) и следует (П.5).

Полагая теперь

$$
g_{i}(t)=\frac{\overline{\eta_{\beta_{i}}(t)}}{\sum_{i=1}^{\infty} \lambda_{i}\left|\eta_{\beta_{i}}(t)\right|^{2}}
$$

нетрудно убедиться, что выполнены соотношения (П.3) и (П.4). Лемма доказана.

ЗАМЕчАНИЕ 1. Из соотношений (П.1), (П.13) и (П.14) нетрудно увидеть, что для любого мультиндекса $j$ сушествуют постоянные $\widehat{C}_{j}, \widehat{Q}_{j}$, и $\widehat{P}_{j}$, зависящие только от $q,\left\{Q_{\ell}:|\ell| \leqslant|j|+1\right\}$ (в частности, не зависящие от конкретного выбора семейства $\left\{\eta_{\beta}(t), \beta \in \mathbb{J}\right\}$ со свойствами (П.1) и (П.2)), такие, что

$$
\begin{gathered}
\left|Q_{j}^{*}\right| \leqslant \widehat{Q}_{j}, \\
\left|C_{j}^{*}\right| \leqslant \widehat{C}_{j}\left(\sum_{|\ell| \leqslant|j|+1}\left|C_{\ell}\right|+\frac{1}{c}\right)^{\widehat{P}_{j}} .
\end{gathered}
$$


ЗАмЕчАниЕ 2. Пусть в условиях леммы соотношение (П.2) вьполняется только при $|t|>\delta>0$.

Тогда соотношения (П.3) и (П.4) будут иметь место при $|t|>2 \delta$, причем для любого мультиндекса $j$ сушествуют постоянные $\widehat{C}_{j}, \widehat{Q}_{j}$ и $\widehat{P}_{j}$, зависящие только от $q,\left\{Q_{\ell}:|\ell| \leqslant|j|+1\right\}$ (в частности, не зависящие от чисел $C_{\ell}, c, \delta$ и конкретного выбора семейства $\left\{\eta_{\beta}(t), \beta \in \mathbb{J}\right\}$ со свойствами (П.1) и (П.2)), такие, что

$$
\left|C_{j}^{*}\right| \leqslant \widehat{C}_{j}\left(\sum_{|\ell| \leqslant|j|+1}^{\left|Q_{j}^{*}\right| \leqslant \widehat{Q}_{j},}\left|C_{\ell}\right|+\frac{1}{c}+\frac{1}{\delta}\right)^{\widehat{P}_{j}} .
$$

Действительно, добавим к нашему семейству $\left\{\eta_{\beta}(t), \beta \in \mathbb{J}\right\}$ еше одну функцию $c \omega(t / \delta)$, где "шапочка" $\omega(t)$ обладает следуюшими свойствами:

$$
\omega(t) \in C^{\infty}\left(\mathbb{R}^{n}\right), \quad \omega(t)= \begin{cases}1, & |t|<1 \\ 0, & |t|>2\end{cases}
$$

Считая (без ограничения общности) $0<\delta<1$, нетрудно проверить, что новое семейство (с добавленной “шапочкой”) удовлетворяет всем условиям леммы. Поэтому сушествует счетное множество $I \subset \mathbb{J}$, числа

$$
\lambda_{0}>0, \quad \lambda_{i}>0, \quad \sum_{i=0}^{\infty} \lambda_{i}=1,
$$

индексы $\beta_{i} \in I$ и функции $\left\{g_{i}(t) \in C^{\infty}\left(\mathbb{R}^{n}\right), i=0,1, \ldots\right\}$ такие, что выполнены соотношения (П.3) и

$$
1=\sum_{i=1}^{\infty} \lambda_{i} \eta_{\beta_{i}}(t) g_{i}(t)+\lambda_{0} c \omega\left(\frac{t}{\delta}\right) g_{0}(t) .
$$

Согласно предыдущему замечанию для $g_{i}(t), i=0,1, \ldots$, вьполнены оценки (П.15) и (П.16), из которых (и еше из (П.19)) и следуют соотношения (П.17) и (П.18).

ЗАмечАниЕ 3. Лемма П.1 останется справедливой, если вместо семейства функций $\left\{\eta_{\beta}(t), \beta \in \mathbb{J}\right\}$, заданных в $\mathbb{R}^{n}$, рассматривать семейство функций $\left\{\eta_{\beta}(t) \in C^{\infty}(\bar{\Omega}), \beta \in \mathbb{J}\right\}$, где $\Omega$ - произвольная выпуклая область. При этом соотношения (П.1) и (П.2) должны вьполняться в $\bar{\Omega}$. Функции $g_{i}(t), i=1,2, \ldots$, также будут определены в $\bar{\Omega}$ и там же будут выполнены соотношения (П.3) и (П.4) (единственное изменение в доказательстве состоит в том, что в качестве множеств $K_{p}$ теперь нужно рассматривать множества $\overline{T_{p} \backslash T_{p-1}} \cap \bar{\Omega}$ и учесть тот факт, что в выпуклом множестве любые две точки можно соединить отрезком прямой). Отметим, что в качестве $\Omega$ можно взять внутренность выпуклого конуса $\Gamma$ с вершиной в начале координат.

Лемма П.2. Пусть $P(t)$ - многочлен степени $N$ от $n$ переменных $t=$ $\left(t_{1}, \ldots, t_{n}\right)$ такой, что

$$
P(\lambda \bar{e}) \not \equiv 0 \quad \text { при } \quad \lambda>0 \quad \text { и любом } \quad \bar{e}, \quad|\bar{e}|=1 .
$$

Пусть заданьи числа $\lambda_{i}>0, i=0,1, \ldots, N$, попарно не равные друг другу. Тогда существует постоянная $c>0$ такая, что

$$
\inf _{|\bar{e}|=1} \max _{0 \leqslant i \leqslant N}\left|P\left(\varepsilon \lambda_{i} \bar{e}\right)\right| \geqslant c \varepsilon^{N}, \quad \varepsilon<1 .
$$


ДокаЗАтельство. Пусть $P(t)=\sum_{k=0}^{N} P_{k}(t)$, где $P_{k}(t)$ - однородные многочлены степени $k$. Из условия (П.20) вытекает, что на единичной сфере $\{\bar{e}:|\bar{e}|=1\}$ все они одновременно в нуль не обрашаются, а потому

$$
\|\bar{P}(\bar{e})\| \geqslant c_{1}>0, \quad|\bar{e}|=1,
$$

здесь и далее $\|\cdot\|$ - евклидова норма вектора, а вектор $\bar{P}(\bar{e})=\left(P_{0}(\bar{e}), P_{1}(\bar{e}), \ldots\right.$, $\left.P_{N}(\bar{e})\right)$. Положим

$$
\begin{aligned}
& A_{0}(\bar{e}, \varepsilon)=P\left(\varepsilon \lambda_{0} \bar{e}\right)=P_{0}(\bar{e})+\varepsilon \lambda_{0} P_{1}(\bar{e})+\left(\varepsilon \lambda_{0}\right)^{2} P_{2}(\bar{e})+\cdots+\left(\varepsilon \lambda_{0}\right)^{N} P_{N}(\bar{e}), \\
& A_{N}(\bar{e}, \varepsilon)=P\left(\varepsilon \lambda_{N} \bar{e}\right)=P_{0}(\bar{e})+\varepsilon \lambda_{N} P_{1}(\bar{e})+\left(\varepsilon \lambda_{N}\right)^{2} P_{2}(\bar{e})+\cdots+\left(\varepsilon \lambda_{N}\right)^{N} P_{N}(\bar{e}) .
\end{aligned}
$$

Полагая вектор $\bar{A}(\bar{e}, \varepsilon)=\left(A_{0}(\bar{e}, \varepsilon), \ldots, A_{N}(\bar{e}, \varepsilon)\right)$ и обозначая через $W(\varepsilon)$ матрицу Вандермонда

$$
W(\varepsilon)=\left(\begin{array}{ccccc}
1 & \left(\varepsilon \lambda_{0}\right) & \left(\varepsilon \lambda_{0}\right)^{2} & \ldots & \left(\varepsilon \lambda_{0}\right)^{N} \\
1 & \left(\varepsilon \lambda_{1}\right) & \left(\varepsilon \lambda_{1}\right)^{2} & \ldots & \left(\varepsilon \lambda_{1}\right)^{N} \\
\vdots & \vdots & \vdots & \ddots & \vdots \\
1 & \left(\varepsilon \lambda_{N}\right) & \left(\varepsilon \lambda_{N}\right)^{2} & \ldots & \left(\varepsilon \lambda_{N}\right)^{N}
\end{array}\right),
$$

имеем $\bar{A}(\bar{e}, \varepsilon)=W(\varepsilon) \bar{P}(\bar{e})$. Отсюда и из $($ П.22) получаем

$$
0<c_{1} \leqslant\|\bar{P}(\bar{e})\| \leqslant\left\|W^{-1}(\varepsilon)\right\|\|\bar{A}(\bar{e}, \varepsilon)\| .
$$

Далее, имеем

$$
\|\bar{A}(\bar{e}, \varepsilon)\| \geqslant \frac{c_{1}}{\left\|W^{-1}(\varepsilon)\right\|} .
$$

Если мы покажем, что $\left\|W^{-1}(\varepsilon)\right\| \leqslant$ const $/ \varepsilon^{N}$, то из (П.23) и будет следовать соотношение (П.21).

Получаем

$$
\left\|W^{-1}(\varepsilon)\right\| \leqslant C_{2} \max _{0 \leqslant i, k \leqslant N}\left|w_{i, k}\right|,
$$

где $w_{i, k}$ - элементы матрицы $W^{-1}(\varepsilon)$. Обозначая теперь через $M_{i, k}$ соответствуюшие миноры матрицы Вандермонда $W(\varepsilon)$, имеем

$$
\begin{aligned}
\left|w_{i, k}\right| & =\left|\frac{M_{k, i}}{\operatorname{det} W(\varepsilon)}\right|=\left|\frac{\varepsilon^{1+2+\cdots+(k-1)+(k+1)+\cdots+N} v_{i, k}\left(\lambda_{0}, \ldots, \lambda_{N}\right)}{\varepsilon^{1+2+\cdots+N} \operatorname{det} W\left(\lambda_{0}, \ldots, \lambda_{N}\right)}\right| \\
& =\frac{1}{\varepsilon^{k}} C(\lambda) \leqslant \frac{C(\lambda)}{\varepsilon^{N}},
\end{aligned}
$$

где $C(\lambda)$ не зависит от $\varepsilon$. Лемма доказана.

ЛЕмма П.3. Пусть $\mathbb{B}$ - банахово и $\mathscr{L}$ - локально выпуклое пространства, причем $\mathbb{B} \subset \mathscr{L}, f(\xi) \in \mathbb{L}(\mathscr{S} \rightarrow \mathscr{L})$ и $\omega(x) \in \mathscr{S}\left(\mathbb{R}^{n}\right)$. Пусть при каждом $y \in(0,1]$ функция $L_{f}^{\omega}(x, y)$ измерима по $x \in \mathbb{R}^{n}$ как функция со значениями в $\mathbb{B}$ и существуют $C$, , а и $b$ такие, что при $0<y \leqslant 1$

$$
\left\|L_{f}^{\omega}(x, y)\right\|_{\mathbb{B}} \leqslant \frac{C}{y^{b}}(1+|x|)^{a} \quad \text { nри n.в. } \quad x \in \mathbb{R}^{n} .
$$

Тогда для любых $\chi(x) \in \mathscr{S}$ и $y \in(0,1] \quad(\widetilde{f}(t), \widetilde{\chi}(t) \widetilde{\omega}(y t)) \in \mathbb{B} u$

$$
\|(\widetilde{f}(t), \widetilde{\chi}(t) \widetilde{\omega}(y t))\|_{\mathbb{B}} \leqslant \frac{C}{y^{b}} \int(1+|x|)^{a}|\chi(x)| d x
$$

для некоторих $C_{1}$ и $N$, не зависящих от банахова пространства $\mathbb{B}$ и обобщенной функции $f$. 
ДоКАЗАТЕЛьСтво. Для любой $\chi(x) \in \mathscr{S}$ в силу (П.24) существует интеграл Бохнера $\int \chi(x) y^{b} L_{f}^{\omega}(x, y) d x \in \mathbb{B}$ и справедлива оценка

$$
\begin{aligned}
\left\|\int \chi(x) y^{b} L_{f}^{\omega}(x, y) d x\right\|_{\mathbb{B}} & \leqslant \int|\chi(x)| y^{b}\left\|L_{f}^{\omega}(x, y)\right\|_{\mathbb{B}} d x \\
& \leqslant C \int(1+|x|)^{a}|\chi(x)| d x
\end{aligned}
$$

С другой стороны, имеем

$$
\begin{aligned}
\int \chi(x) y^{b} L_{f}^{\omega}(x, y) d x & =\int \chi(x) y^{b}\left(f(\xi), \frac{1}{y^{n}} \omega\left(\frac{x-\xi}{y}\right)\right) d x \\
& =\int \chi(x)\left(\widetilde{f}(t), e^{i x t} y^{b} \widetilde{\omega}(y t)\right) d x
\end{aligned}
$$

Здесь мы в последнем равенстве перешли к преобразованию Фурье, учитывая формулу

$$
\left(f(\xi), \frac{1}{y^{n}} \omega\left(\frac{x-\xi}{y}\right)\right)=\left(F[f(\xi)](t), F\left[\frac{1}{y^{n}} \omega\left(\frac{x-\xi}{y}\right)\right](t)\right)=\left(\widetilde{f}(t), e^{i x t} \widetilde{\omega}(y t)\right) .
$$

Теперь покажем, что всюду по $y$

$$
\int \chi(x)\left(\widetilde{f}(t), e^{i x t} y^{b} \widetilde{\omega}(y t)\right) d x=\left(\widetilde{f}(t), \int \chi(x) e^{i x t} y^{b} \widetilde{\omega}(y t) d x\right)
$$

как элементы из пространства $\mathscr{L}$.

Для этого достаточно показать, что для любой $\psi \in \mathscr{L}^{*}$

$$
\left\langle\int \chi(x)\left(\widetilde{f}(t), e^{i x t} y^{b} \widetilde{\omega}(y t)\right) d x, \psi\right\rangle=\left\langle\left(\widetilde{f}(t), \int \chi(x) e^{i x t} y^{b} \widetilde{\omega}(y t) d x\right), \psi\right\rangle .
$$

Так как в (П.29) слева стоит интеграл Бохнера в $\mathbb{B}$, а $\psi \in \mathscr{L}^{*} \subset \mathbb{B}^{*}$ (напомним, что $\mathbb{B} \subset \mathscr{L})$, то

$$
\left\langle\int \chi(x)\left(\widetilde{f}(t), e^{i x t} y^{b} \widetilde{\omega}(y t)\right) d x, \psi\right\rangle=\int \chi(x)\left\langle\left(\widetilde{f}(t), e^{i x t} y^{b} \widetilde{\omega}(y t)\right), \psi\right\rangle d x .
$$

Рассмотрим выражение справа в (П.29). Разобьем $\mathbb{R}^{n}$ на $n$-мерные кубы $\triangle_{j}$ со стороной $\triangle, x_{j} \in \triangle_{j}$. Тогда при фиксированном разбиении интегральные суммы $\sum_{j} \chi\left(x_{j}\right) e^{i x_{j} t} y^{b} \widetilde{\omega}(y t) \triangle^{n}$ сходятся в $\mathscr{S}$, а при $\triangle \rightarrow 0$ они в $\mathscr{S}$ сходятся к $\int \chi(x) e^{i x t} y \widetilde{\omega}(y t) d x$. Поэтому в $\mathscr{L}$

$$
\left(\widetilde{f}(t), \sum_{j} \chi\left(x_{j}\right) e^{i x_{j} t} y^{b} \widetilde{\omega}(y t) \triangle^{n}\right) \underset{\triangle \rightarrow 0}{\longrightarrow}\left(\widetilde{f}(t), \int \chi(x) e^{i x t} y^{b} \widetilde{\omega}(y t) d x\right) .
$$


Следовательно,

$$
\begin{aligned}
\left\langle\left(\widetilde{f}(t), \int \chi(x) e^{i x t} y^{b} \widetilde{\omega}(y t) d x\right), \psi\right\rangle & =\left\langle\lim _{\triangle \rightarrow 0}\left(\widetilde{f}(t), \sum_{j} \chi\left(x_{j}\right) e^{i x_{j} t} y^{b} \widetilde{\omega}(y t) \triangle^{n}\right), \psi\right\rangle \\
& =\lim _{\triangle \rightarrow 0}\left\langle\left(\widetilde{f}(t), \sum_{j} \chi\left(x_{j}\right) e^{i x_{j} t} y^{b} \widetilde{\omega}(y t) \triangle^{n}\right), \psi\right\rangle \\
& =\lim _{\triangle \rightarrow 0} \sum_{j} \chi\left(x_{j}\right)\left\langle\left(\widetilde{f}(t), e^{i x_{j} t} y^{b} \widetilde{\omega}(y t)\right), \psi\right\rangle \triangle^{n} \\
& =\int \chi(x)\left\langle\left(\widetilde{f}(t), e^{i x t} y^{b} \widetilde{\omega}(y t)\right), \psi\right\rangle d x .
\end{aligned}
$$

Сравнивая (П.30) и (П.31), получаем равенство (П.29), в котором слева стоит элемент из $\mathbb{B}$. Следовательно, и элемент справа

$$
\left(\widetilde{f}(t), \int \chi(x) e^{i x t} y^{b} \widetilde{\omega}(y t) d x\right)=\left(\widetilde{f}(t), \widetilde{\chi}(t) y^{b} \widetilde{\omega}(y t)\right) \in \mathbb{B}
$$

Продолжая (П.27), получаем

$$
\int \chi(x) y^{b} L_{f}^{\omega}(x, y) d x=\left(\widetilde{f}(t), \widetilde{\chi}(t) y^{b} \widetilde{\omega}(y t) d y\right)
$$

Отсюда и из (П.26) имеем соотношение (П.25).

ЗАмечАниЕ. Из (П.25) непосредственно следует, что

$$
\|(\widetilde{f}(t), \widetilde{\chi}(t) \widetilde{\omega}(y t))\|_{\mathbb{B}} \leqslant \frac{\text { const }}{y^{b}} \mathscr{P}_{N}[\widetilde{\chi}(t)]
$$

для некоторых постоянных $b$ и $N$.

ЛЕмма П.4. Пусть $\mathbb{B}$ - банахово пространство, $\rho(k)$ - автомодельная функция, $\omega(x) \in \mathscr{S}, f \in \mathbb{L}(\mathscr{S} \rightarrow \mathbb{B})$ u

$$
\sup _{k>1} \frac{1}{\rho(k)}\left\|L_{f}^{\omega}\left(\frac{x}{k}, \frac{y}{k}\right)\right\|_{\mathbb{B}} \leqslant \frac{A}{y^{d}}, \quad|x|^{2}+y^{2}=1, \quad y>0
$$

для некоторых $A$ и $d$. Тогда существуют $A_{1}, a_{1}$ и $b_{1}$ такие, что

$$
\sup _{k>1} \frac{1}{\rho(k)}\left\|L_{f}^{\omega}\left(\frac{x}{k}, \frac{y}{k}\right)\right\|_{\mathbb{B}} \leqslant \frac{A_{1}(1+|x|)^{a_{1}}}{y^{b_{1}}}, \quad x \in \mathbb{R}^{n}, \quad 0<y \leqslant 1
$$


ДокАЗАТЕЛЬСтво. Пусть $\alpha$ - порядок автомодельности $\rho(k)$. Положим

$$
x=k_{0} x^{0}, \quad y=k_{0} y^{0}, \quad\left|x^{0}\right|^{2}+\left(y^{0}\right)^{2}=1, \quad k_{0}^{2}=|x|^{2}+y^{2} .
$$

Рассмотрим два случая.

Случай $1: k_{0} \leqslant 1$. Пользуясь свойствами автомодельных функций (1.10), имеем

$$
\begin{gathered}
\sup _{k>1} \frac{1}{\rho(k)}\left(\left\|L_{f}^{\omega}\left(\frac{x}{k}, \frac{y}{k}\right)\right\|_{\mathbb{B}}\right)=\sup _{k>1} \frac{\rho\left(k / k_{0}\right)}{\rho(k)} \frac{1}{\rho\left(k / k_{0}\right)}\left\|L_{f}^{\omega}\left(\frac{x^{0}}{k / k_{0}}, \frac{y^{0}}{k / k_{0}}\right)\right\|_{\mathbb{B}} \\
\leqslant C_{\varepsilon}\left(\frac{1}{k_{0}}\right)^{\alpha+\varepsilon} \sup _{k>k_{0}} \frac{1}{\rho\left(k / k_{0}\right)}\left\|L_{f}^{\omega}\left(\frac{x^{0}}{k / k_{0}}, \frac{y^{0}}{k / k_{0}}\right)\right\|_{\mathbb{B}} \leqslant C_{\varepsilon}\left(\frac{1}{k_{0}}\right)^{\alpha+\varepsilon} \frac{A}{\left(y^{0}\right)^{d}} \\
\leqslant C_{\varepsilon} k_{0}{ }^{d-\alpha-\varepsilon} \frac{A}{y^{d}} \leqslant C_{1} \frac{\left(|x|^{2}+y^{2}\right)^{(d-\alpha-\varepsilon) / 2}}{y^{d}} \leqslant C_{2} \frac{\left(|x|^{2}+1\right)^{(d-\alpha-\varepsilon) / 2}}{y^{d}} .
\end{gathered}
$$

Откуда и следует (П.33) в этом случае.

Случай $2: k_{0}>1$. Пользуясь (1.10), а также общей оценкой класса (1.9), имеем

$$
\begin{aligned}
\sup _{k>1} & \frac{1}{\rho(k)}\left\|L_{f}^{\omega}\left(\frac{x}{k}, \frac{y}{k}\right)\right\|_{\mathbb{B}} \\
& =\sup _{k>k_{0}} \frac{\rho\left(k / k_{0}\right)}{\rho(k)} \frac{1}{\rho\left(k / k_{0}\right)}\left\|L_{f}^{\omega}\left(\frac{x^{0}}{k / k_{0}}, \frac{y^{0}}{k / k_{0}}\right)\right\|_{\mathbb{B}}+\sup _{1<k<k_{0}} \frac{1}{\rho(k)}\left\|L_{f}^{\omega}\left(\frac{x}{k}, \frac{y}{k}\right)\right\|_{\mathbb{B}} \\
& \leqslant c_{\varepsilon}\left(\frac{1}{k_{0}}\right)^{\alpha-\varepsilon} \frac{A}{\left(y^{0}\right)^{d}}+\sup _{1<k \leqslant k_{0}} C_{\varepsilon} \frac{1}{k^{\alpha-\varepsilon}} C_{1} \frac{(1+|x / k|)^{a}}{(y / k)^{b}} \\
& \leqslant C_{3} \frac{\left(|x|^{2}+1\right)^{(|d|+|\alpha|+\varepsilon) / 2}}{y^{d}}+C_{4} k_{0}^{|b|+|\alpha|+\varepsilon} \frac{(1+|x|)^{a}}{y^{b}} \\
& \leqslant C_{5} \frac{(1+|x|)^{a_{2}}}{y^{d}}+C_{6} \frac{(1+|x|)^{a_{3}}}{y^{b}} .
\end{aligned}
$$

В последних неравенствах использованы соотношения (П.34). Отсюда и следует соотношение (П.33) в этом случае. Лемма доказана.

ЗАмечАниЕ 1 . Лемма остается справедливой, если $k$ заменить на $1 / k$, т.е. если в условиях леммы вместо (П.32) выполнено соотношение

$$
\sup _{k>1} \frac{1}{\rho(k)}\left\|L_{f}^{\omega}(k x, k y)\right\|_{\mathbb{B}} \leqslant \frac{A}{y^{d}}, \quad|x|^{2}+y^{2}=1, \quad y>0
$$

то найдутся $A_{1}, a_{1}$ и $b_{1}$ такие, что

$$
\sup _{k>1} \frac{1}{\rho(k)}\left\|L_{f}^{\omega}(k x, k y)\right\|_{\mathbb{B}} \leqslant \frac{A_{1}(1+|x|)^{a_{1}}}{y^{b_{1}}}, \quad x \in \mathbb{R}^{n}, \quad 0<y \leqslant 1 .
$$

Доказательство аналогично рассмотренному выше.

ЗАмечАниЕ 2. Утверждения леммы (а также замечание 1 к ней) останутся справедливыми, если вместо условия (П.32) требовать

$$
\sup _{k>1} \frac{1}{\rho(k)}\left\|L_{f}^{\omega}\left(\frac{x}{k}, \frac{1}{k}\right)\right\|_{\mathbb{B}} \leqslant A(1+|x|)^{a}, \quad x \in \mathbb{R}^{n},
$$

соответственно вместо (П.35) требовать

$$
\sup _{k>1} \frac{1}{\rho(k)}\left\|L_{f}^{\omega}(k x, k)\right\|_{\mathbb{B}} \leqslant A(1+|x|)^{a}, \quad x \in \mathbb{R}^{n} .
$$


ЛЕмма П.5. Пусть задано семейство $\left\{P_{q}(x), q=0,1, \ldots, d\right\}$, әде $P_{q}(x)-$ однородный многочлен степени $q$ (либо тоэсдественный нуль), и функиия $\varphi(x) \in \mathscr{S}$. Тогда

$$
\varphi(t) \in \widetilde{S}_{\left[d ; P_{0}, P_{1}, \ldots, P_{d}\right]}
$$

в том и только том случае, если

$$
\int Q(x) \varphi(x) d x=0
$$

для любого многочлена степени не более чем $d$ такого, что

$$
P_{q}\left(\frac{\partial}{\partial x}\right) Q(x)=0, \quad q=0,1, \ldots, d
$$

ДоказАТЕЛЬство. Необходимость. Пусть выполнено (П.39) и $Q(x)$ - многочлен степени не более чем $d$ такой, что справедливо соотношение (П.41). Для $h(t) \in \mathscr{S}$ и $h(t)=1$ в некоторой окрестности нуля имеем

$$
\begin{aligned}
\int Q(x) \varphi(x) d x= & \left(Q\left(i \frac{\partial}{\partial t}\right) \delta(t), \widetilde{\varphi}(t)\right)=\left(Q\left(i \frac{\partial}{\partial t}\right) \delta(t), \widetilde{\varphi}(t)-T_{\widetilde{\varphi}}^{d}(t) h(t)\right) \\
& +\left(Q\left(i \frac{\partial}{\partial t}\right) \delta(t), T_{\widetilde{\varphi}}^{d}(t) h(t)\right)=\left(Q\left(i \frac{\partial}{\partial t}\right) \delta(t), T_{\widetilde{\varphi}}^{d}(t)\right)
\end{aligned}
$$

Здесь учтено, что $\widetilde{\varphi}(t)-T_{\widetilde{\varphi}}^{d}(t) h(t)$ имеет в начале координат нуль порядка больше $d$. Так как по условию $T_{\widetilde{\varphi}}^{d}(t)=\sum_{0 \leqslant q \leqslant d} P_{q}(t) \Lambda_{q}(t)$, где $\Lambda_{q}(t)$ - многочлены степени не выше $d-q$, то

$$
\begin{aligned}
\left(Q\left(i \frac{\partial}{\partial t}\right) \delta(t), T_{\widetilde{\varphi}}^{d}(t)\right) & =\sum_{q=0}^{d}\left(Q\left(i \frac{\partial}{\partial t}\right) \delta(t), P_{q}(t) \Lambda_{q}(t) h(t)\right) \\
& =\sum_{q=0}^{d}\left(P_{q}(t) Q\left(i \frac{\partial}{\partial t}\right) \delta(t), \Lambda_{q}(t) h(t)\right) \\
& =\sum_{q=0}^{d}\left(P_{q}\left(-i \frac{\partial}{\partial x}\right) Q(x), F^{-1}\left[\Lambda_{q}(t) h(t)\right](x)\right)=0
\end{aligned}
$$

Отсюда и из (П.42) следует (П.40).

Достаточность. Пусть $\varphi \in \mathscr{S}$ такова, что для любого $Q_{\tau}(x)$ - однородного многочлена степени $\tau \leqslant d$ такого, что выполнено (П.41), имеет место и соотношение (П.40). Имеем

$$
0=\int Q_{\tau}(x) \varphi(x) d x=\left(Q_{\tau}\left(i \frac{\partial}{\partial t}\right) \delta(t), \widetilde{\varphi}(t)\right)=\left(Q_{\tau}\left(i \frac{\partial}{\partial t}\right) \delta(t), T_{\widetilde{\varphi}}^{d}(t)\right)
$$

Наше утверждение вытекает теперь из следуюшей леммы. 
Лемма П.6. Пусть $T(t)$ - многочлен степени не более чем $d$ и задано семейство $\left\{P_{q}(t), q=0,1, \ldots, d\right\}$, где $P_{q}(t)$ - однородный многочлен степени $q$ (либо тождественный нуль). Тогда для того чтобы

$$
T(t)=\sum_{q=0}^{d} P_{q}(t) \Lambda_{d-q}(t)
$$

где $\Lambda_{d-q}(t)$ - многочлен степени не более чем $d-q$, необходимо и достаточно, чтобы

$$
\left(Q_{\tau}\left(\frac{\partial}{\partial t}\right) \delta(t), T(t)\right)=0
$$

для любого однородного многочлена $Q_{\tau}(x)$ степени $\tau \leqslant d$ такого, что

$$
P_{q}\left(\frac{\partial}{\partial x}\right) Q_{\tau}(x)=0
$$

ДокаЗАтельство. Необходимость очевидна. Докажем достаточность. Без ограничения общности можно считать, что $T(t)$ - однородный многочлен степени $d$ $\left(\mathrm{a} \Lambda_{d-q}(t)\right.$ - однородный многочлен степени $\left.d-q\right)$. Обозначим через $\mathscr{P}_{d}$ множество всех многочленов степени не более чем $d$. Пусть

$$
V(t)=\sum_{j=0}^{d} V_{j}(t)=\sum_{|\alpha| \leqslant d} v_{\alpha} t^{\alpha}, \quad U(t)=\sum_{j=0}^{d} U_{j}(t)=\sum_{|\alpha| \leqslant d} u_{\alpha} t^{\alpha}
$$

где $V_{j}$ и $U_{j}$ - однородные многочлены степени $j$. Положим

$$
\begin{aligned}
\langle V(t), U(t)\rangle & =\left(V\left(\frac{\partial}{\partial t}\right) \delta(t), \bar{U}(t)\right)=\left(\sum_{|\alpha| \leqslant d} v_{\alpha} \delta^{(\alpha)}(t), \sum_{|\beta| \leqslant d} \bar{u}_{\beta} t^{(\beta)}\right) \\
& =\sum_{|\alpha| \leqslant d} \alpha ! v_{\alpha} \bar{u}_{\alpha} .
\end{aligned}
$$

Тем самым $\mathscr{P}_{d}-$ конечномерное гильбертово пространство. Рассмотрим в нем линейный оператор

$$
A U(t)=\sum_{q=0}^{d} P_{q}(t) U_{d-q}(t)
$$

Для доказательства леммы достаточно показать, что $T(t) \in \mathscr{R}(A)$. Для этого необходимо и достаточно, чтобы $T(t)$ был ортогонален $\operatorname{Ker}\left(A^{*}\right)$. Теперь утверждение леммы следует из того, что

$$
A^{*} V(x)=\sum_{\ell=0}^{d} \bar{P}_{d-\ell}\left(\frac{\partial}{\partial x}\right) V_{d}(x)
$$


Действительно, имеем

$$
\begin{aligned}
\langle A U(t), V(t)\rangle & =\overline{\langle V(t), A U(t)\rangle}=\sum_{q=0}^{d}\left(\bar{V}\left(\frac{\partial}{\partial t}\right) \delta(t), P_{q}(t) U_{d-q}(t)\right) \\
& =\sum_{q=0}^{d}\left(P_{q}\left(-i \frac{\partial}{\partial x}\right) U_{d-q}\left(-i \frac{\partial}{\partial x}\right) \delta(x), \bar{V}_{d}(i x)\right) \\
& =\sum_{q=0}^{d}\left(U_{d-q}\left(-i \frac{\partial}{\partial x}\right) \delta(x), P_{q}\left(i \frac{\partial}{\partial x}\right) \bar{V}_{d}(i x)\right) \\
& =\sum_{q=0}^{d}\left\langle U_{d-q}(x), \bar{P}_{q}\left(\frac{\partial}{\partial x}\right) V_{d}(x)\right\rangle=\sum_{\ell=0}^{d}\left\langle U_{\ell}(x), \bar{P}_{d-\ell}\left(\frac{\partial}{\partial x}\right) V_{d}(x)\right\rangle .
\end{aligned}
$$

Отсюда следует, что $\ell$-я проекция оператора $A^{*}$ есть $\left(A^{*} V\right)_{\ell}=\bar{P}_{d-\ell}(\partial / \partial x) V_{d}(x)$. Откуда и вытекает (П.46). Лемма П.6, а вместе с ней и лемма П.5 доказаны.

\section{Список литературы}

1. Дрожжинов Ю.Н., Завьялов Б.И. Тауберовы теоремы для обобщенных функций со значениями в банаховых пространствах // Изв. РАН. Сер. матем. 2002. Т. 66. № 4 . C. $47-119$.

2. Дрожжинов Ю.Н., Завьллов Б.И. Об одной многомерной теореме тауберова типа для обобщенных функций со значениями в банаховых пространствах // Докл. РАН. 2003. T. 391. № 2. С. 158-161.

3. Владимиров В.С., Дрожсжинов Ю.Н., Завьялов Б.И. Многомерные тауберовы теоремы для обобщенных функций. М.: Наука, 1986.

4. Сенета Е. Правильно меняющиеся функции. М.: Наука, 1985.

5. Дрожжинов Ю. Н., Завьялов Б. И. Теоремы тауберова типа для обобшенной мультипликативной свертки // Изв. РАН. Сер. матем. 2000. Т. 64. № 1. С. 37-94.

6. Мазья В. Г. Пространства С. Л. Соболева. Ленинград: Изд-во ЛГУ, 1985.

Математический институт им. В. А. Стеклова РАН

E-mail: drozzin@mi.ras.ru, bzavial@mi.ras.ru
Поступила в редакцию

05.05.2003 